\title{
Topological Strings and Integrable Hierarchies
}

\section{Citation}

Aganagic, Mina, Robbert Dijkgraaf, Albrecht Klemm, Marcos Mariño, and Cumrun Vafa. 2005. "Topological Strings and Integrable Hierarchies." Communications in Mathematical Physics 261 (2): 451-516. https://doi.org/10.1007/s00220-005-1448-9.

\section{Permanent link}

http://nrs.harvard.edu/urn-3:HUL.InstRepos:41385009

\section{Terms of Use}

This article was downloaded from Harvard University's DASH repository, and is made available under the terms and conditions applicable to Other Posted Material, as set forth at http:// nrs.harvard.edu/urn-3:HUL.InstRepos:dash.current.terms-of-use\#LAA

\section{Share Your Story}

The Harvard community has made this article openly available.

Please share how this access benefits you. Submit a story.

\section{Accessibility}


hep-th/0312085

UW/PT-03-33

ITFA-2003-58

CERN-TH/2003-290

MAD-TH-03-5

HUTP-03/A083

\title{
Topological Strings and Integrable Hierarchies
}

\author{
Mina Aganagic, ${ }^{a}$ Robbert Dijkgraaf, ${ }^{b}$ Albrecht Klemm, ${ }^{c}$ Marcos Mariño, ${ }^{d}$ and Cumrun Vafa ${ }^{e}$ \\ ${ }^{a}$ Department of Physics, University of Washington at Seattle \\ Seattle, WA 98195-1560, USA \\ ${ }^{b}$ Institute for Theoretical Physics \& Korteweg-de Vries Institute for Mathematics \\ University of Amsterdam, 1018 XE Amsterdam, The Netherlands \\ ${ }^{c}$ Physics Department, University of Wisconsin at Madison \\ Madison, WI 53706-1390, USA \\ ${ }^{d}$ Theory Division, CERN, Geneva 23, CH-1211 Switzerland \\ e Jefferson Physical Laboratory, Harvard University \\ Cambridge, MA 02138, USA
}

\begin{abstract}
We consider the topological B-model on local Calabi-Yau geometries. We show how one can solve for the amplitudes by using $\mathcal{W}$-algebra symmetries which encodes the symmetries of holomorphic diffeomorphisms of the Calabi-Yau. In the highly effective fermionic/brane formulation this leads to a free fermion description of the amplitudes. Furthermore we argue that topological strings on Calabi-Yau geometries provide a unifying picture connecting non-critical (super)strings, integrable hierarchies, and various matrix models. In particular we show how the ordinary matrix model, the double scaling limit of matrix models, and Kontsevich-like matrix model are all related and arise from studying branes in specific local Calabi-Yau three-folds. We also show how A-model topological string on $\mathbf{P}^{1}$ and local toric threefolds (and in particular the topological vertex) can be realized and solved as B-model topological string amplitudes on a Calabi-Yau manifold.
\end{abstract} December 2003 


\section{Introduction}

Topological strings on Calabi-Yau threefolds have served as a unifying theme of many aspects of string theory. They are a rather simple class of theories which relate to many different aspects of string theory, including computing F-terms for superstring compactifications to four dimensions and equivalence with non-critical strings. Many deep phenomena in string theory have a simpler and better understood description in the context of topological strings, in particular large $N$ transitions that encode the connections between gauge theory and geometry.

The aim of this paper is to study the topological B-model on a special class of noncompact Calabi-Yau threefolds and develop various techniques to solve it completely. In the process of doing this we end up unifying a number of different areas of string theory. In particular we will see that the topological B-model on CY backgrounds is the right language to understand various properties of non-critical strings (see [1] for a review of non-critical bosonic strings). For example it has been known that non-critical bosonic strings have two different matrix model descriptions: a double scaling limit of a matrix model, in which the string world-sheets emerge through the 't Hooft ribbon diagrams as triangulations, as well as a finite $N$ matrix model, introduced by Kontsevich, in which the matrix diagrams can be considered as open string field theory diagrams that triangulate moduli space. It has also been known that Virasoro (or more generally $\mathcal{W}$-algebra) constraints essentially characterize the amplitudes. We will see that all these viewpoints are naturally understood and unified into a single setting through the topological B-model on Calabi-Yau threefolds.

We will be mainly considering Calabi-Yau threefolds which are non-compact and can be viewed as a hypersurface

$$
z w-H(p, x)=0,
$$

where $z, w \in \mathbf{C}$ and we consider cases where $x, p \in \mathbf{C}$ or $\mathbf{C}^{*}$. One has a well defined string theory of B-model on the corresponding Calabi-Yau geometry. We can then study B-model in the target space of this geometry. This will give the Kodaira-Spencer theory of gravity [2]. The observables of this theory (the modes of the 'tachyon field' in terms of the two-dimensional non-critical string) correspond to variations of the complex structure at infinity. In each patch this is described by the modes of a chiral boson $\phi(x)$, defined by

$$
\partial \phi(x)=p(x) .
$$


Quantum consistency of the Kodaira-Spencer theory of variation of complex structure gives rise to Virasoro or more generally W-constraints. These identities in turn completely fix the string amplitudes. The basic idea is very simple: The symmetries of the B-model involve holomorphic diffeormorphisms which preserve the equation of Calabi-Yau (1.1) as well as the holomorphic 3 -form

$$
\Omega=\frac{d z}{z} \wedge d x \wedge d p
$$

(this is analogous to the symmetries of $c=1$ strings [3]). If we turn off $H(p, x) \rightarrow 0$ the symmetries of the B-model will be enhanced. In fact arbitrary symplectic diffeomorphisms of the $(x, p)$ plane (which by definition preserve $d x \wedge d p$ ) is the symmetry of this theory as it now preserves (1.1). This is generated by arbitrary functions $f(x, p)$ by the symplectic flow. In the quantum theory this is the $\mathcal{W}$-algebra symmetry. Shifting the background back to $H \neq 0$ breaks some of this symmetry. However, the broken generators of this symmetry corresponds to "Goldstone Bosons" which get identified with the "tachyon fields" which deform the complex structure of the B-model. These symmetries are strong enough, as we shall see in this paper, to completely fix the amplitudes.

The connection of the B-model to matrix model comes from two different directions, both related to the B-branes: One is in the context of compact branes leading to large $N$ transitions; the other is for non-compact branes, leading to relation to Kontsevich like matrix model with source terms determining the position of the non-compact brane.

For compact branes, as has been argued before [4, [5] the topological B-model for the class of CY given by (1.1) is a large $N$ dual to a matrix model, where $p(x)$ is identified with the spectral density of the matrix, $x$ with the eigenvalue, and $H(p, x)=0$ is the large $N$ limit of the loop equations. The way this arises is by considering Calabi-Yau geometries with compact B-branes wrapped over blown up $\mathbf{P}^{1}$ geometries and using the response of the complex structure to the B-brane, which leads to a flux for the holomorphic 3-form $\Omega$ over the three-cycle surrounding it. In particular if one is interested in having functions of the form

$$
H(p, x)=p^{r}+x^{s}+\text { lower order terms }
$$

then one can take an $A_{r-1}$ quiver matrix theory (with adjoint matrices at the $r-1$ nodes and bifundamental for edges) and tune the potential for the adjoints suitably. The exact finite N-loop equations for the matrix quiver theories can be derived using conformal field theory techniques [6,5, [7]. In the large $N$ limit, with suitable tuning of these parameters, one can end up with a function with the lower terms vanishing. This gets identified with 
non-critical bosonic string with the background corresponding to the $(r, s)$ minimal model. Note that this double scaling limit is not necessary to obtain a smooth string theory. It is only necessary if one wants to get a specific non-critical bosonic string. The topological string is a smooth string theory and does make sense for arbitrary $H(p, x)$. The quadratic case where

$$
H(x, p)=p^{2}-x^{2}-\mu
$$

corresponds to the $c=1$ non-critical bosonic string at self-dual radius [8].

On the other hand the variations of the complex structure at infinity can also be induced by addition of non-compact branes to the geometry [9]. This is the topological string analog of the back-reaction of the branes on gravity. This in particular implies that we should be able to get the amplitudes of the closed string theory upon variation of the complex structure at infinity, by introducing open string sectors corresponding to branes and integrating this sector out. The open string field theory on these branes turns out to be a Kontsevich-like matrix model whose classical action can be read off from the Calabi-Yau geometry:

$$
W=\frac{1}{g_{s}} \operatorname{Tr}\left[\int P(X) d X-\Lambda X\right]
$$

with $P(X)$ obtained by solving $H(p, x)=0$ and $P=\Lambda$ give the classical position of the branes. The case $(r, s)=(1,2)$ gives the usual Kontsevich model with action

$$
W=\frac{1}{g_{s}} \operatorname{Tr}\left[\frac{1}{3} X^{3}-\Lambda X\right] .
$$

From this point of view it is natural to compute the change in the closed string partition function, as a result of the back reaction to the presence of $N$ branes, as a rank $N$ matrix integral. This explains why a closed string theory can be written both in terms of a large $N$ and a finite $N$ matrix model, as it was the case for pure topological gravity.

Another interesting example we will study is given by the case where $x, p$ are cylindrical variables and

$$
H(p, x)=e^{p}+e^{x}+1
$$

This is the B-model mirror [10] relevant for the topological vertex studied in [9]. We will show that the full topological vertex can be computed directly from the perspective of the target space gravity.

In all these models one can also use the brane perspective for formulating and solving the $\mathcal{W}$ identities. Non-compact branes turn out to be fermions of the KS theory [9] in 
terms of which the $\mathcal{W}$ constraints are bilinear relations. This perspective not only gives a natural choice for certain normal ordering ambiguities, but also leads to a very simple solution: In terms of fermions the theory is free (i.e. the corresponding state is an element of KP hierarchy).1 However these are not ordinary free fermions: The fermions $\psi(x)$ are not globally well-defined geometrical objects on the Riemann surface $H(p, x)=0$. First of all, they are related to the chiral scalar through the usual bosonization formula

$$
\psi(x)=e^{\phi(x) / g_{s}}
$$

which is not defined globally but has a natural interpretation as wave functions defined on patches of the Riemann surface. We find that the fermions in various patches transform to one another not geometrically but rather by Fourier-type transforms dictated by viewing $H(x, p)=0$ as a subspace of the quantum mechanical $x, p$ phase space. Even though we do not have a deep explanation of this fact, we can motivate it and check it in all the examples considered here. The fact that branes transform as wavefunctions rather than as ordinary geometric objects on the Riemann surface seems to be related to holomorphic anomaly for topological strings [2] and its interpretation in terms of choice of polarization of quantum mechanical system, as in the closed string context [11] as we will sketch at the end of this paper. The fermionic formulation turns out to be a powerful viewpoint which also leads to a unifying simple solution to the quantum amplitudes. This encompasses not only the fact that fermions lead to the simplest description of the $c=1$ non-critical bosonic string amplitudes [12], but also for the topological vertex.

From the viewpoint of Calabi-Yau geometry the complexity of the model will be related to the number of asymptotic infinities in the geometry of the Riemann surface $H(p, x)=0$. For example in the case of the $(1, r)$ Virasoro minimal model, $H=x-p^{r}$ coupled to gravity, we have only one asymptotic infinity (represented by $x \rightarrow \infty$ ). For the $c=1$ string at selfdual radius and the mirror of topological string on $\mathbf{P}^{1}$ (which we relate to specific CalabiYau threefold geometry) we have two asymptotic infinities given by $(p \pm x) \rightarrow \infty$. For the topological vertex we have three asymptotic regions $x \rightarrow \infty, p \rightarrow \infty,(x \sim p+i \pi) \rightarrow \infty$. In general when we have $k$ boundaries the amplitudes of the theory are captured by a state in the $k$-fold tensor product of a chiral boson, whose positive frequency modes represent the deformations of the complex structure at infinity of the corresponding patch. These states

1 This generalizes the situation for $c=1$ non-critical bosonic string where the amplitudes only involve phase multiplication of fermions. 
will be the tau-function of some hierarchy captured by the corresponding $\mathcal{W}$-constraints. The brane correlations are given by suitable ("quantum") Wick contractions of the free fermions.

The organization of this paper is as follows: In section 2 we discuss the general setup of non-compact Calabi-Yau geometries and branes of interest. In section 3 we discuss deformations of complex structure on this class of Calabi-Yau and the quantum KodairaSpencer theory in this context. In section 4 we discuss the back reaction of B-branes on complex geometry, and identification of non-compact B-branes as fermions. We also discuss the action on the branes and how this can be used to compute closed string amplitudes. We consider two distinct ways this can be done depending on whether one is using compact (leading to ordinary matrix models) or non-compact B-branes (leading to Kontsevich-like matrix models). In section 5 we present a number of examples and solve each one viewed from closed string (i.e. $\mathrm{KS}$ ) viewpoint, large $N$ duality viewpoint and Kontsevich-like matrix model view point. We will focus on four classes of examples: $(r, s)$ minimal models coupled to bosonic strings, $c=1$ bosonic string, topological string on $\mathbf{P}^{1}$, and finally the topological vertex:

$$
\begin{array}{lllll}
(i) & H(p, x)=p^{r}+x^{s}+\ldots & \leftrightarrow & (r, s) \text { minimal models coupled to gravity } \\
(i i) & H(p, x)=p^{2}-x^{2} & \leftrightarrow & c=1 \text { self dual radius coupled to gravity } \\
(\text { iii }) & H(p, x)=e^{p}+q e^{-p}+x & \leftrightarrow & \text { Mirror of topological string on } \mathbf{P}^{1} \\
(i v) & H(p, x)=e^{p}+e^{x}+1 & \leftrightarrow & \text { Mirror of } \mathbf{C}^{3} \text { (topological vertex) }
\end{array}
$$

In section 6 we comment on connections to non-critical (super)strings. In section 7 we end with some open questions and concluding remarks. In appendix A we collect some facts about the $c=1$ scattering amplitudes and in appendix B we discuss some amplitudes of the topological vertex as solutions to $\mathcal{W}$-algebra constraints.

\section{Topological strings on non-compact Calabi-Yau geometries}

In this paper we consider B-model topological strings on non-compact Calabi-Yau manifold $X$ given as a hypersurface in $\mathbf{C}^{4}$

$$
z w-H(p, x)=0
$$


These geometries allow a Ricci-flat metric that is conical at infinity [13]. The holomorphic $(3,0)$ form $\Omega$ can in this case be chosen to be

$$
\Omega=\frac{d z \wedge d p \wedge d x}{z}
$$

One should consider B-model topological string theory as a quantization of the variation of complex structures on $X$ [2]. If one only considers perturbation of the function $H(p, x)$, keeping the dependence on $w, z$ fixed, the problem reduces essentially to one (complex) dimension. In that case it helps to consider the Calabi-Yau $X$ as a fibration over the $(p, x)$-plane, with fiber the rational curve $z w-H=0$. This fiber clearly degenerates - it develops a node — on the locus

$$
H(p, x)=0 .
$$

This degeneration locus is therefore an affine non-compact curve.

Moreover the periods of $\Omega$ over three-cycles on $X$ reduce, by Cauchy's theorem, to integrals of the two-form

$$
\int_{D} d x \wedge d p
$$

over (real two-dimensional) domains $D$ in the complex two-dimensional $(p, x)$-plane, such that $\partial D \subset \Sigma$, where $\Sigma$ is the analytic curve $H(p, x)=0$. These integrals in turn reduce by Stokes' theorem (coordinate patch by coordinate patch) to

$$
\int_{\gamma} p d x
$$

where $\gamma=\partial D$ denotes a one-cycle on the Riemann surface $\Sigma$. Thus the complex structure deformations of the function $H(p, x)$ are controlled by the one-form

$$
\lambda=p d x
$$

This one-form is holomorphic in the interior of $\Sigma$, but will have in general singularities at infinity.

There are a natural set of 2-branes (with a world-volume that has complex dimension one) in this geometry [14]. They are parameterized by a fixed point $\left(p_{0}, x_{0}\right)$ in the $(p, x)$ plane, and are given by the subspace

$$
(z, w, p, x)=\left(z, w, p_{0}, x_{0}\right)
$$


where $(z, w)$ are restricted by

$$
z w=H\left(p_{0}, x_{0}\right) .
$$

That is, the brane wraps the fiber of the fibration of $X$ over the $(p, x)$ plane. This thus gives us a one complex dimensional subspace which generically we can identify, say, with the coordinate $z$. For simplicity we drop the 0 subscript from $p_{0}, x_{0}$ and denote them simply by $(p, x)$.

The worldvolume theory on these branes is given by a reduction of holomorphic ChernSimons down to two (real) dimensions. Its kinetic action will involve the term [14:

$$
\frac{1}{g_{s}} \int d z d \bar{z} p \bar{\partial} x
$$

From this we see that in the brane probe the holomorphic symplectic form $d p \wedge d x$ gives rise to field variables $p(z)$ and $x(z)$ which should be considered as canonically conjugated. In fact, from the action we read off that their zero modes, which can be identified with the coordinates of the moduli space of these branes, will have canonical commutation relation

$$
[x, p]=g_{s} .
$$

This Lagrangian structure and its quantum appearance will play a key role in this paper.

There is a more restricted class of branes, which will be very important for us. These correspond to fixing $\left(p_{0}, x_{0}\right)$ to lie on the Riemann surface $C$, that is on the degeneration locus

$$
H\left(p_{0}, x_{0}\right)=0 .
$$

In this case the equation satisfied for $(z, w)$ becomes

$$
z w=0,
$$

and we can choose either $z=0$ or $w=0$ to satisfy it. In particular the brane in the 'bulk,' i.e. at arbitrary position $\left(p_{0}, x_{0}\right)$, now splits to two intersecting branes on the boundary given by $H=0$. Taking any of these two branes, we can move it along the points of the Riemann surface. In other words, the moduli of either of these branes is now given by a one complex dimensional space that can be identified with the points on the Riemann surface $H=0$. There is a sense in which these two types of branes 'annihilate' one another. What we mean by this is that, as discussed in [14], the branes in the bulk give rise to zero amplitudes in the topological B-model. It is only after they split up on the Riemann surface that they contribute to B-model amplitudes. In this sense we can think of these as being 'brane/anti-brane' pairs.2

2 This is not strictly true as we can define the notion of anti-brane for either of these two branes to be the same brane geometry but counting the brane number by a (-1) factor, as in [15. 


\section{Classical and Quantum Complex Structure Deformation}

We will be considering the B-model topological strings on non-compact Calabi-Yau geometries which quantize the complex structure. Including the full $g_{s}$ corrections this corresponds to the quantum Kodaira-Spencer theory of gravity [2].

At tree-level the B-model computes the variation of complex structure through period integrals. In the case of a compact Calabi-Yau $X$ one picks a canonical basis of three-cycles $\left(A_{i}, B_{i}\right)$ of $H_{3}(X)$ and compute the periods

$$
s_{i}=\oint_{A_{i}} \Omega, \quad \mathcal{F}_{i}=\oint_{B_{i}} \Omega .
$$

These periods are not independent, but satisfy the special geometry relation

$$
\mathcal{F}_{i}(s)=\frac{\partial \mathcal{F}_{0}}{\partial s_{i}}
$$

in terms of the prepotential $\mathcal{F}(s)$. Mathematically these identities express the fact that the image under the period map of the (extended) moduli space of $X$ forms a Lagrangian submanifold of $H^{3}(X, \mathbf{C})$.

Quantum Kodaira-Spencer theory associates to these data, using the concepts of geometric quantization, a quantum wave function $|X\rangle$ [11, 16]. In the full quantum theory the $A$ and $B$ periods become canonically conjugate operators, with commutation relations

$$
\left[\oint_{A_{i}} \Omega, \oint_{B_{j}} \Omega\right]=g_{s}^{2} \delta_{i j}
$$

The closed string coupling $g_{s}^{2}$ plays here the role of $\hbar$. The topological string partition function should be considered as the wave function of the quantum state in the coordinate basis of the $A$-cycle periods $s_{i}$

$$
Z(s)=\langle s \mid X\rangle .
$$

In this way the tree-level prepotential $\mathcal{F}_{0}(s)$ gets replaced by the full string partition function, including all the higher genus quantum corrections

$$
Z(s)=\exp \mathcal{F}(s), \quad \mathcal{F}(s)=\sum_{g} g_{s}^{2 g-2} \mathcal{F}_{g}(s) .
$$

In the WKB approximation the $B$-cycle periods, that play the role of dual momenta to the $A$-cycle periods $s_{i}$, are given by

$$
\oint_{B_{i}} \Omega=g_{s}^{2} \frac{\partial \mathcal{F}}{\partial s_{i}} \sim \frac{\partial \mathcal{F}_{0}}{\partial s_{i}} .
$$


All of this is highly reminiscent of the way chiral blocks in two-dimensional CFT are described.

The CY geometries we consider in this paper are non-compact and are given by the hypersurface

$$
z w-H(p, x)=0
$$

In particular we will be considering complex deformations of the Calabi-Yau involving varying $H(p, x)$ only. We will consider situations where the complex structure of the curve $H=0$ can be changed at 'infinity' only. This is of course not generally the case, for example when $H$ defines a higher genus Riemann surface, which has normalizable moduli. In such cases we decompose the Riemann surface to pants and apply our constructions below to each of the components, and deal with the more general situation by the gluing constructions.

Taking this into account, we thus consider situations where $H=0$ corresponds to a genus 0 surface with a number of boundaries. Near each of the boundaries we choose a local coordinate $x$ such that $x \rightarrow \infty$ at the boundary. We also choose a symplectic completion $p(x)$ such that the canonical one-form can be written as

$$
\lambda=p d x .
$$

We now want to consider variations of the complex structure at this infinity $x=\infty$. To this end we introduce a scalar field $\phi(x)$ such that

$$
\lambda=\partial \phi .
$$

This we do at each boundary component. In other words, we have the relation $p(x)=\partial_{x} \phi$. Classically, we choose the expectation value

$$
\langle\phi(x)\rangle=\phi_{c l}(x)
$$

such that

$$
\partial_{x} \phi_{c l}=p_{c l}(x)
$$

where $p_{c l}(x)$ is obtained by solving the relation $H\left(p_{c l}(x), x\right)=0$ near the point $x=\infty$.

If we consider $H(p, x)$ as a Hamiltonian function on a phase space $(p, x)$, the complex curve $\Sigma$ given by $H=0$ is the level set of fixed energy and gets an interpretation as a Fermi surface in the corresponding fermion theory. The one-form $\lambda$ is nothing but the 
Liouville form $p d x$. From this point of view the equation $p_{c l}=\partial_{x} \phi$ is just the standard Hamilton-Jacobi relation that gives the classical action $\phi(x)$ for a solution with $H=0$.

The field $\phi(x)$ should be considered as a chiral bosonic scalar field, and arbitrary chiral deformations of it correspond to complex deformations of the surface near $x=\infty$. When we consider the most general deformation of the complex structure of the surface near this point, according to the Kodaira-Spencer picture, this is equivalent to a reidentification of $p$ and $x$. Namely we can consider a general Laurent expansion around $x=\infty$ of the form

$$
p(x)=\partial \phi(x)=p_{c l}(x)+t_{0} x^{-1}+\sum_{n>0} n t_{n} x^{n-1}+\sum_{n>0} \mathcal{F}_{n} x^{-n-1} .
$$

(Note that in some of our examples $x$ is a periodic variable, in which case we consider $e^{n x}$ as the expansion series instead of the powers $x^{n}$, i.e. we make a Fourier expansion instead of a Laurent expansion.) Since the space is non-compact near $x=\infty$, there are no restrictions on the coefficients $t_{n}$. Since they multiply the non-normalizable modes of $\partial \phi$ they should be considered as boundary values, or in the language of the AdS/CFT correspondence, as coupling constants. (The zero-mode $t_{0}$, which is log-normalizable and has no conjugated partner, should be discussed separately, as we will.)

However the coefficients $\mathcal{F}_{n}$ that multiply the normalizable modes, that are irrelevant at $x \rightarrow \infty$, are expected to be fixed by the rest of the Riemann surface data. In fact, viewing $\phi(x)$ as a quantum field, the positive and negative frequency modes are not independent. In particular they are conjugate variables, with commutator proportional to $g_{s}^{2}$ coming from Kodaira-Spencer action, just as was the case for the compact periods that we discussed before. We can therefore view (in leading order in $\left.g_{s}\right) \mathcal{F}_{n}$ as

$$
\mathcal{F}_{n}=g_{s}^{2} \frac{\partial \mathcal{F}}{\partial t_{n}}
$$

where $\mathcal{F}\left(t_{n}^{i}\right)$ is the free energy of the theory (which at tree-level is given by $\Phi \sim \mathcal{F}_{0} / g_{s}^{2}$ ). Here we consider the free energy as a function of the infinite set of couplings $t_{n}^{i}$, where $i=1, \ldots, k$ runs over the number of boundary components and $n \geq 0$. More precisely, in the full quantum theory the coefficients $\mathcal{F}_{n}$ are realized as the dual operators

$$
\mathcal{F}_{n}=g_{s}^{2} \frac{\partial}{\partial t_{n}}
$$

Equivalently, we can think of the free energy $\mathcal{F}$ as defining a state $|V\rangle$ in the Hilbert space $\mathcal{H}^{\otimes k}$, where $\mathcal{H}$ denotes the Hilbert space of a single free boson and $k$ is the number 
of asymptotic infinities. The consistency of the existence of a unique function $\mathcal{F}$ and the consistency of these expansion in each patch completely fixes $\mathcal{F}$ as we will discuss later in this section.

This is best understood in terms of coherent states. If we introduce the standard mode expansion of a chiral boson

$$
\partial \phi(x)=\sum_{n \in \mathbf{Z}} \alpha_{n} x^{-n-1}, \quad\left[\alpha_{n}, \alpha_{m}\right]=n g_{s}^{2} \delta_{n+m, 0}
$$

then the coherent state $|t\rangle$ is defined as

$$
|t\rangle=\exp \left(\sum_{n>0} t_{n} \alpha_{-n}\right)|0\rangle .
$$

In this representation we have, with $k$ boundary components, the relation

$$
\exp \mathcal{F}\left(t^{1}, \ldots, t^{k}\right)=\left\langle t^{1}\right| \otimes \cdots \otimes\left\langle t^{k} \mid V\right\rangle
$$

Note that in this representation of the state $|V\rangle \in \mathcal{H}^{\otimes k}$ as a coherent state wave function, the annihilation and creation operators $\alpha_{ \pm n}$ are represented as

$$
\alpha_{-n}=n t_{n}, \quad \alpha_{n}=g_{s}^{2} \frac{\partial}{\partial t_{n}} .
$$

\subsection{Framing and the $\mathcal{W}$ algebra}

Before discussing how $\mathcal{F}$ can be determined by this consistency condition, we will discuss the notion of choice of coordinate, or what we will call framing.

Given an asymptotic point $x \rightarrow \infty$, and the choice of the one-form $\lambda=p d x$, we can ask how unique this choice of $\lambda$ is? We can in particular consider coordinate transformations of the form

$$
x \rightarrow x+f(p)
$$

keeping $p$ fixed. These are canonical transformations in the sense that the pair $(p, x+f(p))$ is still symplectic conjugated. This type of change of variables we call framing.

Consider a general analytic expansion of $f(p)$,

$$
f(p)=\sum_{n \geq 0} a_{n} p^{n} .
$$


For a quantum chiral scalar any change of the local coordinate $\delta x=\epsilon(x)$ is implemented by the operator

$$
\oint \epsilon(x) T(x) d x
$$

acting on the Hilbert space $\mathcal{H}$, where

$$
T(x)=\frac{1}{2}(\partial \phi)^{2}
$$

is the energy momentum tensor. In the case at hand we have

$$
\epsilon(x)=f(p(x))=\sum_{n \geq 0} a_{n} p^{n}(x)=\sum_{n \geq 0} a_{n}(\partial \phi)^{n}
$$

thus the corresponding quantum operator that implements this framing is given by

$$
\frac{1}{2} \sum_{n \geq 0} a_{n} \oint d x(\partial \phi)^{n+2}=\sum_{n \geq 0} a_{n} W_{0}^{n+2},
$$

which is given by the linear combination of zero modes of a $\mathcal{W}_{1+\infty}$ algebra. In one of the examples relevant for this paper (the topological vertex) the $x$ and $p$ are periodic variables and the only non-trivial framing deformation is of the form

$$
f(p)=r p
$$

where $r$ is an integer. This leads to the action of the zero mode of the $W^{3}$ generator on the Hilbert space,

$$
W_{0}^{3}=\oint d x(\partial \phi)^{3},
$$

as we will note later. . $^{3}$ Notice that this operator, when written as a differential operator on coherent wave state functions, is the "cut and join" operator considered for example in [19].

3 The relevance of this $\mathcal{W}_{\infty}$ algebra in the context of $\mathcal{N}=2$ gauge theory has been noted in 17]. Its modification on the topological vertex as turning on arbitrary Casimirs on the edges as propagators has been noted by [18]. 


\subsection{Broken $\mathcal{W}$-symmetry and Ward identities}

We will now turn to the general philosophy that we will use in this paper to determine the full partition function in the relevant examples. The starting point here is the underlying symmetry of the problem. From the point of view of the three-dimensional Calabi-Yau $X$ these symmetries are obviously given by the global diffeomeorphisms that preserve the choice of the holomorphic volume-form $\Omega$ (or equivalently the choice of complex structure). When we consider the reduction the two dimensions, these symmetries are implemented as diffeomorphisms of the $(p, x)$ plane that preserve the symplectic form or holomorphic area $d p \wedge d x$. Equivalently they are therefore given by general holomorphic canonical transformations of the phase space variables $(p, x)$.

The corresponding Lie algebra of infinitesimal transformations is given by the infinitedimensional algebra $\mathcal{W}_{1+\infty}$. The Hamiltonian vector fields that generate these transformations can be locally identified with general polynomials $f(p, x)$. The infinitesimal action of these Hamiltonians is just

$$
\delta x=\frac{\partial f(p, x)}{\partial p}, \quad \delta p=-\frac{\partial f(p, x)}{\partial x} .
$$

(Subsequently, we will often assume these canonical transformation to be linear transformations, i.e., to be $S p(2, \mathbf{R}) \cong S L(2, \mathbf{R})$ transformations, but that is not necessary at this point in the discussion.)

However these symmetry considerations do not yet take into account the presence of the curve

$$
H(p, x)=0
$$

which is the locus where the fibration over the $(p, x)$ plane degenerates. Only if $H$ vanishes identically (or is a constant), the full symplectic diffeomorphism group will act as unbroken symmetries. If $H$ is not identically zero, most of this symmetry group will be broken. A typical transformation will deform the level set $H(p, x)=0$ and therefore will generate a deformation of the complex structure. In fact, the Kodaira-Spencer field $\phi(x)$ can be viewed as the "Goldstone boson" for these broken symmetries.

Typically we have one Goldstone boson for each broken symmetry. Here, the story is slightly different: We consider the Cartan subalgebra of $\mathcal{W}_{1+\infty}$ and realize it as the analog of "Goldstone bosons". In the $x$-patch these correspond to generators $x^{n}$, giving the $t_{n}$ generators. The rest of the generators of $\mathcal{W}_{1+\infty}$ are not independent and can be 
written in terms of the corresponding $t_{n}$ modes. For example, another natural Cartan subalgebra given by generators $p^{n}$, which as we discussed in the last section are identified with the framing, can be written in terms of the zero modes of $(\partial \phi)^{n}$. This is similar to the situation considered in [20] where some spacetime symmetries are broken and only a subset of broken symmetries are realized as Goldstone bosons (the simplest example being a flat D-brane which breaks both translational and rotational symmetries but only translational symmetries are realized as massless Goldstone bosons on it).

Precisely because of this realization of the symmetry algebra we have a huge left-over constraint: One for each generator of the $\mathcal{W}_{1+\infty}$, which are far more than the Cartan generators. Apart from this fact, the situation is just as in other physical applications: even though the symmetry group is broken, the broken symmetries are still important they give rise to Ward identities that can be used to constrain (and sometimes even solve) the scattering amplitudes of the Goldstone bosons. This will be the approach that we take in this paper. We will implement the Ward identities of the broken $\mathcal{W}$-symmetries and thus solve for the full string free energy $\mathcal{F}(t)$ as a function of the coupling constants or deformation parameters $t_{n}$ at the boundaries. We will now sketch the general features of this approach, leaving details to later sections when we discuss explicit examples and when can use the full power of the reformulation in terms of D-branes.

Note that the subgroup of $\mathcal{W}_{1+\infty}$ of the form $H(p, x) f$ for any $f$ will still be a symmetry even after we shift the vacuum. This is responsible, as we will discuss later for the fact that the brane amplitudes are annihilated by $H(p, x)$.

Let us first consider the classical (tree-level) situation. So we ignore all $g_{s}$ effects. We consider deformations of the curve $H(p, x)=0$, where we allow singularities at the various points at infinity. We parameterize these points at infinity with local coordinates $\left\{x_{i}=\infty\right\}$, with $i=1, \ldots, k$ and $k$ the number of boundary components. At each of these points we have an expansion of the KS field of the form

$$
p_{i}=\partial \phi_{i}=p_{i}^{c l}\left(x_{i}\right)+t_{0}^{i} x_{i}^{-1}+\sum_{n>0} n t_{n}^{i} x_{i}^{n-1}+g_{s}^{2} \sum_{n>0} \frac{\partial \mathcal{F}}{\partial t_{n}^{i}} x_{i}^{-n-1}
$$

for a single, global function $\mathcal{F}\left(t_{n}^{1}, \ldots, t_{n}^{k}\right)$.

The local symplectic coordinates $\left(p_{i}, x_{i}\right)$ around the points $x_{i}=\infty$ are related by canonical transformations, as we will see in more detail in a moment. For a two-dimensional phase space general canonical transformations are just area-preserving diffeomorphisms. Of course there is at each base point $x=\infty$ a local framing ambiguity, given by the Cartan 
subalgebra of $W_{1+\infty}$ that fixes the conjugate variable $p$. This is captured by the action of the generators on the local coherent state $\left|t^{i}\right\rangle$, as we discussed in the previous section.

Now consider the action of a $\mathcal{W}_{1+\infty}$ element generated by a Hamiltonian $f$. In local coordinates $\left(p_{i}, x_{i}\right)$ this Hamiltonian is given by the function $f\left(p_{i}, x_{i}\right)$. Let us assume that it is of the form

$$
f\left(p_{i}, x_{i}\right)=p_{i}^{n} x_{i}^{m}
$$

This element implements the transformation

$$
\delta x_{i}=n p_{i}^{n-1} x_{i}^{m}, \quad \delta p_{i}=-m p_{i}^{n} x_{i}^{m-1}
$$

Ignoring all quantum normal ordering ambiguities, which we will return to after we discuss branes and fermions in the next section, this generator is implemented in the KS theory as the mode $W_{m}^{n+1}$ of the $\mathcal{W}$-current

$$
W^{n+1}(x) \sim \frac{1}{n+1}(\partial \phi)^{n+1}
$$

namely

$$
W_{m}^{n+1}=\oint_{\mathcal{C}_{i}} x_{i}^{m} W^{n+1}(x) \sim \oint_{\mathcal{C}_{i}} x_{i}^{m} \frac{1}{n+1}(\partial \phi)^{n+1}
$$

Here the contour $\mathcal{C}_{i}$ encloses the given puncture. When written in terms of the local parameters $t_{n}^{i}$ these $\mathcal{W}$-generators act as order $n+1$ differential operators. Particularly relevant is the case of the spin one current $W^{1}=\partial \phi$. Its modes correspond to the Hamiltonians $f(p, x)=x^{m}$. They generate the pure deformations of the field $\partial \phi(x)=p$

$$
\delta x=0, \quad \delta p=m x^{m-1} .
$$

That is, they generate (locally) the linear flows $\partial / \partial t_{n}^{i}$.

In this way we get a local action of the $\mathcal{W}$-algebra at each puncture. But there is one very important and non-trivial relation between all these actions: they should all parameterize the deformations of the same analytic curve. That is, if we introduce a deformation (3.8) at the puncture $P_{i}=\left\{x_{i}=\infty\right\}$, this will induce deformations at all other punctures $P_{j}=\left\{x_{j}=\infty\right\}$. The deformed functions $p_{j}\left(x_{j}\right)$ which are given in terms of the local expansions of the Kodaira-Spencer field $\phi$ should all describe, in different coordinate patches of course, one and the same analytic curve. 
This condition relates the various local actions in terms of a global Ward identity. This identity takes the symbolic form

$$
\left\langle\sum_{i} \oint_{C_{i}} W\right\rangle=0
$$

Equivalently, one can think of deforming the contour $\mathcal{C}_{i}$ around $P_{i}$ over the Riemann surface into contours $\mathcal{C}_{j}$ encircling the other punctures $P_{j}$. We claim that this Ward identity is sufficient to solve for the partition function. However, in order to do so, we first have to relate the mode expansion of the $\mathcal{W}$ currents in the local patches. For this we have to know how the Kodaira-Spencer field $\phi(x)$ transforms from patch to patch.

\subsection{Canonical transformations and Kodaira-Spencer field}

For simplicity let us assume there are two punctures with local coordinates $x$ and $\widetilde{x}$. At each puncture we have the canonical one-form that can be written as $p d x$ respectively $\widetilde{p} d \widetilde{x}$. These two one-forms should coincide up to a gauge transformation, so we have a relation of the form

$$
p d x-\widetilde{p} d \widetilde{x}=d S
$$

This expresses the fact that the pairs $(p, x)$ and $(\widetilde{p}, \widetilde{x})$ are related by a canonical transformation, if we extend the coordinate transformation to the full $(p, x)$ plane. That is, we have

$$
d p \wedge d x=d \widetilde{p} \wedge d \widetilde{x}
$$

That this symplectic two-form is preserved is of course also obvious from the fact that is given by the reduction of the holomorphic three-form of the Calabi-Yau space.

In general such a canonical transformation is given in terms of a generating function $S(x, \widetilde{x})$ that satisfies by definition

$$
p=\frac{\partial S(x, \widetilde{x})}{\partial x}, \quad \widetilde{p}=-\frac{\partial S(x, \widetilde{x})}{\partial \widetilde{x}}
$$

At each puncture we can introduce the local Kodaira-Spencer scalar fields

$$
p(x)=\frac{\partial \phi}{\partial x}, \quad \widetilde{p}(\widetilde{x})=\frac{\partial \widetilde{\phi}}{\partial \widetilde{x}}
$$


If we plug-in this form for $p$ and $\widetilde{p}$ into (3.10) we get

$$
\begin{aligned}
& \frac{\partial \phi(x)}{\partial x}=\frac{\partial S(x, \widetilde{x})}{\partial x}, \\
& \frac{\partial \widetilde{\phi}(\widetilde{x})}{\partial \widetilde{x}}=-\frac{\partial S(x, \widetilde{x})}{\partial \widetilde{x}} .
\end{aligned}
$$

Note that in this formulation the variable $\widetilde{x}$ starts out as a function of both $x$ and $p$. Once we give $p=\partial \phi(x)$ the relation between $\widetilde{x}$ and $x$ is implicitly determined by (3.12). In the final equations the function $S$ should be regarded as given once and for all (it just determines the coordinate transformation) and the two functions $\phi$ and $\widetilde{\phi}$ should be considered as the variables that are expressed into each other.

Each of these fields has a mode expansion of the type (3.8) given (at tree-level) in terms of the genus-zero free energy $\mathcal{F}_{0}$ and the couplings

$$
\begin{aligned}
& \partial \phi(x)=\sum_{n>0} n t_{n} x^{n-1}+\sum_{n>0} \frac{\partial \mathcal{F}_{0}}{\partial t_{n}} x^{-n+1} \\
& \partial \widetilde{\phi}(\widetilde{x})=\sum_{n>0} n \widetilde{t}_{n} \widetilde{x}^{n-1}+\sum_{n>0} \frac{\partial \mathcal{F}_{0}}{\partial \widetilde{t}_{n}} \widetilde{x}^{-n+1} .
\end{aligned}
$$

Given the relation between the fields $\phi(x)$ and $\widetilde{\phi}(\widetilde{x})$ we can clearly obtain in this way constraints on the free energy $\mathcal{F}_{0}(t, \widetilde{t})$.

It is interesting to consider a small fluctuation $\phi_{q u}$ around the classical value $\phi_{c l}$. If we write

$$
\partial \phi=\partial \phi_{c l}+\partial \phi_{q u}
$$

one easily verifiest 1 that the field $\partial \phi_{q u}$ transforms as

$$
\partial \widetilde{\phi}_{q u}(\widetilde{x}) d \widetilde{x}=\partial \phi_{q u}(x) d x
$$

That is, the field $\phi_{q u}$ is a globally defined scalar field on the Riemann surface. Of course for finite values the transformation rules for $\phi_{q u}$ are highly non-linear.

4 To see this, note that expanding (3.12) about the classical solution we have

$$
\partial \phi_{q u}(x)=\frac{\partial^{2} S}{\partial x \partial \widetilde{x}}\left(x, \widetilde{x}_{c l}\right) \delta \widetilde{x}, \quad \partial \widetilde{\phi}_{q u}(\widetilde{x})=-\frac{\partial^{2} S}{\partial x \partial \widetilde{x}}\left(x_{c l}, \widetilde{x}\right) \delta x
$$

where $\delta x, \delta \widetilde{x}$ are the corresponding small fluctuations in $x, \widetilde{x}$. It follows that $\partial \phi_{q u}(x)=$ $-\partial \widetilde{\phi}_{q u}\left(\widetilde{x}_{c l}\right) \frac{\delta \widetilde{x}}{\delta x}$, and since $\widetilde{x}=\widetilde{x}_{c l}(x)+\delta \widetilde{x}=\widetilde{x}_{c l}\left(x_{c l}(\widetilde{x})+\delta x\right)+\delta \widetilde{x}$ implies that $\delta \widetilde{x}=-\frac{\partial x_{c l}}{\partial x} \delta x$, the claim follows. 
We will see in the next section that the correct physical interpretation of equations $(3.12)$ is as the semi-classical $\left(g_{s} \rightarrow 0\right)$ saddle-point approximation of the relation

$$
e^{\widetilde{\phi}(\widetilde{x}) / g_{s}}=\int d x e^{-S(x, \widetilde{x}) / g_{s}} e^{\phi(x) / g_{s}}
$$

We now know, at least at tree-level, how both the coordinate $x$ and the field $\partial \phi=p$ transform from patch to patch. Therefore these formulas can now be used to determine the transformation of the $\mathcal{W}$-currents from patch to patch. From these transformation laws we can then read off the Ward identities and in the end solve for the free energy, as we will demonstrate in many concrete examples later.

\subsection{Linear transformations and quantization}

As a small aside we will make the above formalism a bit more transparent by considering the case of a linear canonical transformation. So let us consider a $S L(2, \mathbf{R}) \cong S p(2, \mathbf{R})$ transformation

$$
\begin{aligned}
& \widetilde{p}=a p+b x \\
& \widetilde{x}=c p+d x
\end{aligned}
$$

with

$$
g=\left(\begin{array}{ll}
a & b \\
c & d
\end{array}\right) \in S p(2, \mathbf{R}), \quad a d-b c=1
$$

Now these relations can be written (for $c \neq 0$ ) as

$$
\begin{aligned}
& p=\frac{1}{c}(-d x+\widetilde{x}) \\
& \tilde{p}=\frac{1}{c}(x+a \widetilde{x})
\end{aligned}
$$

so that the generating function is given by

$$
S(x, \widetilde{x})=\frac{1}{2 c}\left(-d x^{2}+2 x \widetilde{x}-a \widetilde{x}^{2}\right)
$$

In this case one can straightforwardly go to the quantization, where $x$ and $p$ become conjugate variables with $g_{s}$ playing the role of $\hbar$

$$
[x, p]=g_{s} .
$$

We now want to consider these variables as operators acting on wave-functions $\Psi(x)$ with

$$
p=-g_{s} \frac{\partial}{\partial x} \text {. }
$$


As is well-known, linear canonical transformations can be unambiguously carried over to the quantum case. There is an (almost) unique lift of the $S p(2, \mathbf{R})$ element $g$ to a unitary operator $U(g)$ on the quantum mechanical Hilbert space $V$ of $L^{2}$ wave-functions. This representation is known as the metaplectic representation. The metaplectic group $M p(2)$ is a two-fold cover of the symplectic group $S p(2, \mathbf{R})$.

It might be helpful to remind the reader that the metaplectic representation can be considered as the bosonic analogue of the spinor representation of the group $\operatorname{Spin}(n)$, defined as a double cover of $S O(n)$. Indeed, for a given a symmetric form $h_{i j}$ spinors are obtained as representations of the Clifford algebra

$$
\gamma_{i} \gamma_{j}+\gamma_{j} \gamma_{i}=h_{i j}
$$

One then writes half of the $\gamma_{i}$ as fermionic creation operators $\theta_{a}$ and the other half as fermionic annihilation operators $\pi_{a}=\partial / \partial \theta_{a}$. A spinor is then simply a function $\Psi(\theta)$ of these anti-commuting variables. The generators of $\operatorname{Spin}(n)$ are written as anti-symmetric quadratic expressions in the $\theta_{a}$ and $\pi_{a}$.

Similarly, in case of a (non-degenerate) anti-symmetric form $\omega_{i j}$ one now starts with a representation of the Heisenberg algebra

$$
\xi_{i} \xi_{j}-\xi_{i} \xi_{j}=\omega_{i j}
$$

After one picks a polarization in terms of coordinates $x_{a}$ and momenta $p_{a}=\partial / \partial x_{a}$, the representations of this algebra are given by square-integrable wavefunctions $\Psi(x)$. Because the $x_{a}$ are bosonic, this representation is infinite-dimensional, in contrast with the fermionic spinor representations. In complete analogy, the metaplectic representation of the group $M p(n)$, now defined as the double cover of $S p(n)$, is generated by the symmetric quadratic functions in the variables $x_{a}$ and $p_{a}$.

In the two-dimensional case the infinitesimal generators of $S p(2)$ are given by

$$
\begin{aligned}
& J_{+}=x^{2}, \\
& J_{0}=x p+p x=-g_{s}\left\{x, \partial_{x}\right\}, \\
& J_{-}=p^{2}=g_{s}^{2} \partial_{x}^{2} .
\end{aligned}
$$

In the metaplectic representation the kernel of the matrix $U(g)$ is essentially given by the exponential of the generating function $S(x, \widetilde{x})$ that we have just determined. More precisely, including the full $g_{s}$ dependence, we have the transformation law

$$
U(g) \Psi(\widetilde{x})=\int \frac{d x}{\sqrt{2 \pi g_{s} c}} \exp \left[\frac{1}{2 g_{s} c}\left(d x^{2}-2 x \widetilde{x}+a \widetilde{x}^{2}\right)\right] \Psi(x) .
$$


Note that all these integrals should be considered as contour integrals in the complex $x$ plane, where the choice of contour is determined such that the integral makes sense. There is a square-root ambiguity in this action that in the end requires the double-cover.

The case we will use often is when the linear canonical transformation corresponds to the element of $S L(2, \mathbf{Z})$,

$$
S=\left(\begin{array}{cc}
0 & -1 \\
1 & 0
\end{array}\right) \in S L(2, \mathbf{Z})
$$

that interchanges the coordinate and the momentum

$$
\begin{aligned}
& \widetilde{p}=-x, \\
& \widetilde{x}=p .
\end{aligned}
$$

This is of course quantum mechanically implemented by the Fourier transform

$$
U(S) \Psi(\widetilde{x})=\int \frac{d x}{\sqrt{2 \pi g_{s}}} e^{x \widetilde{x} / g_{s}} \Psi(x) .
$$

\section{B-Branes}

Branes have played a key role in a deeper understanding of superstrings. It is thus not surprising that also for topological strings they play a key role. As we will find, in terms of brane degrees of freedom, the topological string amplitudes become very simple.

In the context of superstring target space, branes are defined by their impact on gravitational modes, as sources for certain fields. A similar story is also true for topological string for branes. In particular consider a one complex dimensional subspace inside the Calabi-Yau with $N$ B-branes wrapped over it. Then as discussed in [9] this affects the closed string modes by changing the periods of the holomorphic threeform $\Omega$. Namely, let $C$ be a 3 -cycle linking the B-brane world-volume. Then we have the following change in $\Omega$

$$
\Delta \int_{C} \Omega=N g_{s} .
$$

So the operator that creates a brane shifts the value of the period (and therefore the complex structure) in the dual cycle. Note that this back reaction is invisible at tree-level.

The importance of the branes for us is to use the action on the world-volume of the branes to find the closed string amplitudes. This is the familiar story that integrating out the open string sector gives the closed string results for the deformed geometry. Symbolically we can write this effect as

$$
Z_{\text {closed }}(m) Z_{\text {open }}=Z_{\text {closed }}\left(m^{\prime}\right)
$$


where $m$ denote the closed string moduli of the Calabi-Yau where the branes live and $m^{\prime}$ is the deformed string moduli including the back reaction of the branes. This will depend on the location of the brane, through (4.1). The back reaction can be obtained by integrating out the open string sector. In other words we have

$$
Z_{\text {open }}=Z_{\text {closed }}\left(m^{\prime}\right) / Z_{\text {closed }}(m)
$$

In many cases of interest the full relevant moduli dependence is in $m^{\prime}$ and this becomes an efficient method to compute closed string amplitudes.

We will be considering two kinds of B-branes: B-branes wrapped over (i) compact cycles or (ii) non-compact cycles. In the first case of compact cycles, where we consider branes wrapped on $\mathbf{P}^{1}$ cycles, we get geometric transitions where a $\mathbf{P}^{1}$ shrinks and the Calabi-Yau undergoes the conifold transition with an $S^{3}$ emerging. In that case we obtain a new homology class and a corresponding new period integral. In the non-compact case the geometry of the brane is $\mathbf{C}$ and the geometry of Calabi-Yau is modified at infinity. It this latter case that we will predominantly focus on in this paper. We can also have both types of branes present and we will also briefly comment on that.

\subsection{Compact Branes}

The geometry will contain $\mathbf{P}^{1}$ 's near each of which the CY looks locally like $\mathcal{O}(-1) \oplus$

$\mathcal{O}(-1) \rightarrow \mathbf{P}^{1}$, where the B-brane is wrapped over $\mathbf{P}^{1}$. The three cycle surrounding $\mathbf{P}^{1}$ in this case is an $S^{3}$. The gravity back reacts, as discussed above, by

$$
\int_{S^{3}} \Omega=N g_{s}
$$

This suggests, as was first conjectured in the mirror context in [21] that the Calabi-Yau undergoes a transition where $\mathbf{P}^{1}$ shrinks and $S^{3}$ grows and the size of $S^{3}$ is given by

$$
S=N g_{s}
$$

A class of examples which exhibits this geometry is given by the Calabi-Yau defined as a hypersurface

$$
z w-p^{2}+W^{\prime}(x)^{2}=0
$$

where $W^{\prime}(x)$ is a polynomial of degree $n$ in $x$. Near each critical point $W^{\prime}(x)=0$ we have a conifold singularity which we can blow up to a $\mathbf{P}^{1}$. Around each of the $\mathbf{P}^{1}$ 's we can wrap 
$N_{i}$ B-branes, as $1=1, \ldots, n$. Then as conjectured in [22 this undergoes a transition to a geometry with $n S^{3}$ 's replacing the $n \mathbf{P}^{1}$ 's

$$
z w-p^{2}+W^{\prime}(x)^{2}+f(x)=0
$$

where $f(x)$ is a polynomial of degree $n-1$ in $x$, whose coefficients are fixed by the condition that the size of each $S^{3}$ is

$$
S_{i}=N_{i} g_{s}
$$

In the context of topological string this duality was explored in [4]. In particular the open string field theory was identified with a $2 \mathrm{~d}$ holomorphic Chern-Simons theory which was shown to reduce to a matrix model with action

$$
S=\operatorname{Tr} W(\Phi) / g_{s}
$$

and thus it leads to the conjecture [四]:

$$
Z_{\text {closed }}\left(S_{i}\right)=\int D \Phi \exp \left(-\operatorname{Tr} W(\Phi) / g_{s}\right)
$$

We will explore aspects of this correspondence later in this paper.

\subsection{Non-compact Branes}

Consider non-compact branes that we discussed before, whose moduli are parameterized by a point on the Fermi surface $H(p, x)=0$. In the presence of these branes, (4.1) implies a change in the integral of the 1 -form $\lambda=p d x$, which is the reduction of $\Omega$ (integrated along two of the normal directions). In this reduction the period of $\lambda$, integrated around the point $P$ on the Riemann surface where the brane intersects, receives a non-trivial contribution:

$$
\oint_{P} \lambda=\oint_{P} \partial \phi=g_{s}
$$

Let $\psi(P)$ denote the operator creating a brane at the point $P$ on the surface. Then we have the following identity inside correlation functions

$$
\left\langle\cdots \oint_{P} \partial \phi \psi(P) \cdots\right\rangle=g_{s}\langle\cdots \psi(P) \cdots\rangle
$$

which implies that the brane creation operator affects the closed string sectors by

$$
\psi(z)=\exp \left(\phi(z) / g_{s}\right)
$$


This means that the brane is the fermion associated to $\phi$ by the standard bosonization rules. This fact was pointed out in [9]. Note that (4.6) is also consistent with the fact that the classical action for the fermionic brane at position $z$ is given by [14]

$$
S(z)=\frac{1}{g_{s}} \int^{z} \lambda=\phi(z) / g_{s}
$$

Similarly the anti-brane is defined by the condition that it gives the opposite change in the period integral which means that it is given by the conjugate fermion:

$$
\psi^{*}(z)=\exp \left(-\phi(z) / g_{s}\right)
$$

The action for the anti-brane is negative that of the brane and we have $Z_{\text {brane }}=$ $1 / Z_{\text {anti-brane }}$.

Now consider the Kodaira-Spencer theory in the context of the discussion of the previous section where the relevant geometry is described by the deformation of a Riemann surface $H(p, x)=0$. Let $x \rightarrow \infty$ denote a coordinate for asymptotic infinity of the Riemann surface. Suppose we put branes at positions $x^{i}$ near this asymptotic patch. Then the gravitational backreaction is given by

$$
\prod_{i} \psi\left(x^{i}\right)=\prod_{i} \exp \left(\phi\left(x^{i}\right) / g_{s}\right)=\prod_{i \neq j}\left(x^{i}-x^{j}\right) \exp \left(\sum_{i} \phi\left(x^{i}\right) / g_{s}\right) .
$$

If we compute the expectation value $\langle\partial \phi(x)\rangle$ in this background we find

$$
\langle\partial \phi(x)\rangle=g_{s} \sum_{i} \frac{1}{x-x_{i}}=g_{s} \sum_{n>0} x_{i}^{-n} x^{n-1}
$$

which means that, apart from the prefactor which measures the interaction between the branes, we have turned on a background given by the couplings

$$
t_{n}=\frac{g_{s}}{n} \sum_{i}\left(x^{i}\right)^{-n}
$$

for $n>0$. The momentum of $\phi$ (related to the $t_{0}$ mode) is also shifted by the number of fermions we put in. We could also consider putting anti-branes, which would give a similar formula as above, except that we have

$$
t_{n}=\frac{g_{s}}{n} \sum \pm\left(x^{i}\right)^{-n}
$$


where \pm is correlated with whether we have put a brane or anti-brane at $x^{i}$ and also we get a prefactor of $\left(x^{i}-x^{j}\right)^{ \pm 1}$ depending on whether we put branes of the same type or opposite type at $x^{i}$ and $x^{j}$.

We can now recast the amplitudes in terms of branes. Namely, if we put $N$ branes at positions $x^{1}, \ldots, x^{N}$ in the local $x$-patch, the corresponding correlation function is given by

$$
\left\langle N\left|\prod_{i=1}^{N} \psi\left(x^{i}\right)\right| V\right\rangle=e^{\mathcal{F}(t)} \prod_{i<j}\left(x^{i}-x^{j}\right) .
$$

Here we have considered the case with one asymptotic infinity. If we have $s$ asymptotic infinities, we can consider putting one stack of branes for each asymptotic region and recover $\mathcal{F}$. We can also use this relation to write the state $|V\rangle$ directly in the fermionic basis. We will return to this below.

\subsection{Branes and wave functions}

As we discussed above, inserting a fermion $\psi(x)$ at a point on the Riemann surface corresponds in the Calabi-Yau manifold, to inserting a B-brane there. Since the B-branes are globally well defined objects this would seem to imply that the fermions are free. As we will explain below, this is basically true, however the relation to B-branes implies that the fermions have rather unusual property in going from patch to patch. This was in fact discussed in the previous section and we will restate it here in terms of the wave functions for fermions.

Let us consider the world-volume theory on the brane. In fact, let us insert a B-brane in an asymptotic patch $x_{i} \rightarrow \infty$. Since the $\mathrm{D}$-brane is non-compact the partition function of the D-brane is a wave function $Z_{\text {open }}\left(x_{i}\right)$ depending on $x_{i}$, since this is what is fixed at infinity on the world-volume of the D-brane. Let us denote this by

$$
Z_{\text {open }}\left(x_{i}\right)=\Psi\left(x_{i}\right)
$$

On the other hand, as we have argued above, in the Kodaira-Spencer theory this corresponds to inserting a fermion $\psi=e^{\phi / g_{s}}$ at $x=x_{i}$, i.e. we have

$$
\Psi\left(x_{i}\right)=\left\langle\psi\left(x_{i}\right)\right\rangle
$$

Since $\Psi\left(x_{i}\right)$ is a wave function, it transforms like one in going from patch to patch. 
More precisely, in this patch, we really have a symplectic pair of variables $\left(x_{i}, p_{i}\right)$. In terms of the theory on the B-branes the variables $x_{i}$ and $p_{i}$ correspond to the zero modes of fields that are canonically conjugate, so $\left[p_{i}, x_{i}\right]=g_{s}$. The wave function $\Psi\left(x_{i}\right)$ forms a metaplectic representation of this algebra, and by (4.11), so does $\psi\left(x_{i}\right)$.

Consider another patch with symplectic pair of coordinates $\left(p_{j}, x_{j}\right)$ and the corresponding fermion. Than we have that $\left(p_{i}, x_{i}\right)$ and $\left(p_{j}, x_{j}\right)$ are related by a canonical transformation preserving the symplectic form $d x_{i} \wedge d p_{i}=d x_{j} \wedge d p_{j}$, with a generating function $S\left(x_{i}, x_{j}\right)$. This acts in the usual way on the wave functions of $\Psi_{i}$, so $\psi_{i}\left(x_{i}\right)$ must transform in the same way as well,

$$
\psi_{j}\left(x_{j}\right)=\int d x_{i} e^{-S\left(x_{i}, x_{j}\right) / g_{s}} \psi_{i}\left(x_{i}\right) .
$$

In terms of the bosons $\psi(x)=e^{\phi(x) / g_{s}}$, this is what we anticipated in (3.14).

In particular, if we restrict to linear transformations $g \in S p(2, \mathbf{R})$, we find that the fermion field $\psi(x)$ transforms in the metaplectic representation

$$
\psi_{j}\left(x_{j}\right)=U(g) \psi_{i}\left(x_{i}\right)
$$

This transformation property is immediately clear at tree-level if we make use of the relation to the Kodaira-Spencer field through the back reaction. Using the bosonization/fermionization formulas we see that the one-point function of the brane creation operator

$$
\Psi(x)=\langle\psi(x)\rangle
$$

can be expressed as

$$
\Psi(x)=\exp \frac{1}{g_{s}} \int^{x} p(x) d x=\exp \frac{1}{g_{s}} \phi(x) .
$$

But this is just the WKB approximation for a wave function! The transformation rules for the boson $\phi(x)$ that we found in section 3 therefore immediately imply the transformation rules for the fermion/brane. In fact, these rules become more transparent from the brane perspective. Starting from the linear action of the coordinate transformation in the Hilbert space of brane wave function $\psi(x)$, one derives the non-linear action on the space of Kodaira-Spencer fields $\phi(x)$.

Recall that the boson $\phi(x)$ has a classical piece, corresponding to the background geometry, and a fluctuating quantum piece:

$$
\phi(x)=\phi_{c l}(x)+\phi_{q u}(x)
$$


The classical piece is given by the integral of the canonical one-form on the Riemann surface, $\phi_{c l}(x)=\int^{x} p d x$. It is the field $\phi_{q u}(x)$ that creates the quanta of the KodairaSpencer field. Similarly we have to distinguish the classical contribution to the fermions that create branes/anti-branes

$$
\psi(x)=e^{\phi_{c l}(x) / g_{s}} \psi_{q u}(x), \quad \psi^{*}(x)=e^{-\phi_{c l}(x) / g_{s}} \psi_{q u}^{*}(x) .
$$

Note that only the full expression $\psi(x)$ transforms in the metaplectic representation. So, in all the above formulas one should always subtract the classical contribution to find the transformation rules for the quantum field $\psi_{q u}(x)$.

In particular, in the case of small quantum fluctuations we see that we can approximate (4.12) by a Gaussian integral. This implies that in this approximation $\psi_{q u}$ transforms as

$$
\psi_{j}^{q u}\left(x_{j}\right)\left(d x_{j}\right)^{\frac{1}{2}}=\psi_{i}^{q u}\left(x_{i}\right)\left(d x_{i}\right)^{\frac{1}{2}}
$$

So, in this limit the fermions do transform as actual global spin $1 / 2$ field on the Riemann surface. This fact is well-known in quantum mechanics — wavefunctions transform as half differentials. This is directly related to another fact. The loop momenta

$$
\oint \partial \phi=\oint p d x
$$

of the boson $\phi(x)$ are globally well-defined. The invariance of these periods of the Liouville form under canonical transformations is well-known in classical and quantum mechanics. In our present context they are just the projections of the period of the holomorphic 3-form $\Omega$ on the Calabi-Yau threefold. In terms of the branes/fermion we have

$$
\partial \phi=\psi^{*} \psi
$$

and therefore the periods are given by the "norm" of the wave function

$$
\oint \partial \phi=\int d x \psi^{*}(x) \psi(x)
$$

In order to make sense of this expression in different coordinate patches the fields $\psi, \psi^{*}$ should transform as a half-differential - a fact well-known from quantum mechanics.

Coming back to the one-point function $\Psi(x)=\langle\psi(x)\rangle$, we note that it is not given by an arbitrary wave function. Semi-classically it obviously satisfies the Schrödinger equation

$$
H(p, x) \Psi(x)=0
$$


where the Hamiltonian is given by the equation of the Riemann surface! As discussed before, this is a reflection of the unbroken part of the $\mathcal{W}$-algebra symmetry when we shift to the $H(p, x)$ background. We have found that this fact generalizes in many settings to the full quantum theory. For example, for the topological vertex this fact immediately leads to the quantum dilogarithm as giving the full quantum amplitudes with one set of Lagrangian branes in $\mathbf{C}^{3}$, as we will discuss later. In general however, there are normal ordering ambiguities in writing $H(p, x)$, which make this difficult to use. We will return to this below, where we will provide a way to resolve these normal ordering ambiguities.

\subsection{The quantum free energy $\mathcal{F}$ and B-branes}

In this subsection we explain how one can use the formulation in terms of branes and the $\mathcal{W}_{1+\infty}$ symmetries of the theory to compute the quantum free energy, in terms of an infinite sequence of Ward identities that the amplitude $|V\rangle$ satisfies. This in particular provides the correct normal ordering prescription for the $\mathcal{W}_{1+\infty}$ symmetry generators.

Suppose we pick a patch, say $x_{i} \rightarrow \infty$ corresponding to a point $P_{i}$ on the Riemann surface. In this patch, consider an action of the $\mathcal{W}_{1+\infty}$ generator given by the Hamiltonian

$$
f\left(x_{i}, p_{i}\right)=x_{i}^{m} p_{i}^{n}
$$

Quite generally, such a Hamiltonian is represented in the fermionic representation as

$$
W_{m}^{n+1}=\oint_{P_{i}} \psi^{*}\left(x_{i}\right) x_{i}^{m} p_{i}^{n} \psi\left(x_{i}\right)
$$

As we discussed in section 3 , the $\mathcal{W}$ actions preserve the symplectic form and they generate broken symmetries of the theory corresponding to repameterizations of the Riemann surface, provided their action is regular. This action generalizes the framing ambiguity which also acts as $\mathcal{W}$-algebra in the fermionic basis. Namely [23] the generators are given by fermion bilinears $W_{0}^{n+1}$.

In the case of one puncture, the $\mathcal{W}$ symmetry implies that its generators annihilate the state:

$$
\oint_{P} \psi^{*}(x) x^{m} p^{n} \psi(x)|V\rangle=0 \text {. }
$$

When there is more than one puncture, the symmetry generators do not annihilate the state $|V\rangle$ but get related to shifting of coordinates on other punctures:

$$
\oint_{P_{i}} \psi^{*}\left(x_{i}\right) x_{i}^{m} p_{i}^{n} \psi\left(x_{i}\right)|V\rangle=-\sum_{j \neq i}^{s} \oint_{P_{j}} \psi^{*}\left(x_{j}\right) x_{i}\left(x_{j}, p_{j}\right)^{m} p_{i}\left(x_{j}, p_{j}\right)^{n} \psi\left(x_{j}\right)|V\rangle .
$$


In the above equation, $x_{i}=x_{i}\left(x_{j}, p_{j}\right)$ and $p_{i}=p_{i}\left(x_{j}, p_{j}\right)$ correspond to the canonical transformations of coordinates between the different patches, so (4.14) is simply a consequence of how the fermions transform. In all cases we will restrict to $S L(2, \mathbf{Z})$ transformations. The transformations relating different patches could have been relaxed to arbitrary symplectic transformations, however the normal-ordering ambiguities in terms of defining these in general give rise to many different solutions related by subtle quantum ambiguities, so we will not consider this.

The Ward identities that the symplectic reparameterization imply are sufficient to fix the state $|V\rangle$ (Strictly speaking this is true for Riemann surfaces of genus zero. There is a subtlety here for higher genus Riemann surfaces, to which we will return later). To solve the Ward identities for $|V\rangle$ it is useful to bosonise the fermions, and evaluate (4.14) in an arbitrary coherent state $\langle t|=\left\langle t_{1}\right| \otimes \ldots \otimes\left\langle t_{s}\right|$. This gives rise to a set of differential equations for the free energy, as we have for example,

$$
\begin{aligned}
& \left\langle t\left|\oint_{P_{i}} e^{-\phi\left(x_{i}\right) / g_{s}} x_{i}{ }^{m} p_{i}^{n} e^{\phi\left(x_{i}\right) / g_{s}}\right| V\right\rangle \\
& \quad=\oint_{P_{i}} e^{-\phi\left(x_{i} ; t_{k}, \partial_{k}\right) / g_{s}}\left(g_{s} \partial_{x_{i}}\right)^{n} x_{i}^{m} e^{\phi\left(x_{i} ; t_{k}, \partial_{k}\right) / g_{s}} e^{\mathcal{F}(t)} .
\end{aligned}
$$

These can be solved recursively, genus by genus: expanding (4.14) in power series in $g_{s}$ as well as the free energy, $\mathcal{F}\left(t, g_{s}\right)=\sum_{g} F_{g}(t) g_{s}^{2 g-2}$, one first gets a set of differential equations satisfied by $\mathcal{F}_{0}(t)$. Solving this, at next order $g_{s}^{2}$ order we get a Ward identity for $\mathcal{F}_{1}(t)$ and so on.

We discussed above that the generators of $\mathcal{W}_{1+\infty}$ algebra that give rise to symmetries of the theory correspond to those symplectic coordinate transformations which are regular on the Riemann surface. Note that, in the classical limit - more precisely, in the limit where the Kodaira-Spencer theory becomes an ordinary theory of a globally defined free chiral boson on a Riemann surface - the generators must correspond to symmetries of this classical theory, which are well known. The Ward identities (4.14) reduce in this limit to

$$
\int_{P_{i}} x_{i}^{m} p_{i}\left(x_{i}\right)^{n} \partial \phi\left(x_{i}\right)=-\sum_{j \neq i}^{s} \int_{P_{j}} x_{i}\left(x_{j}\right)^{m} p_{i}\left(x_{j}\right)^{n} \partial \phi\left(x_{j}\right)
$$

where $p_{i}=p_{i}\left(x_{i}\right)$ is the classical equation that $p_{i}$ satisfies on the Riemann surface. This generates a symmetry provided $x_{i}^{m} p_{i}\left(x_{i}\right)^{n}$ are holomorphic on the punctured Riemann surface. In the following sections we will see many examples of how $\mathcal{W}_{1+\infty}$ symmetries can be used to find $|V\rangle$. 
In the cases that we consider in this paper, the state corresponding to KodairaSpencer theory on the Riemann surface with punctures is a Bogoliubov transformation of the fermionic vacuum, corresponding to fermions being free. We conjecture that this is generally the case; it should be possible to derive this from the $\mathcal{W}_{1+\infty}$ constraints.

Introduce the familiar mode expansion of the fermions

$$
\psi\left(x_{i}\right)=\sum_{n \in \mathbf{Z}} \psi_{n+1 / 2}^{i} x_{i}^{-n-1}, \quad \psi^{*}\left(x_{i}\right)=\sum_{n \in \mathbf{Z}} \psi_{n+1 / 2}^{* i} x_{i}^{-n-1}
$$

for $i=1, \ldots s$ where $s$ is the number of punctures, with anticommutation relations

$$
\left\{\psi_{n}^{i}, \psi_{m}^{j *}\right\}=\delta^{i j} \delta_{n+m, 0}
$$

in such a way that the positive modes annihilate the vacuum:

$$
\psi_{n}^{i}|0\rangle=\psi_{n}^{i *}|0\rangle=0, \quad n>0
$$

Then the state $|V\rangle$ can be written as

$$
|V\rangle=\exp \left[\sum_{i, j=1}^{s} \sum_{m, n=0}^{\infty} a_{m n}^{i j} \psi_{-m-1 / 2}^{i} \psi_{-n-1 / 2}^{j *}\right]|0\rangle
$$

Having computed the state $|V\rangle$ we can explicitly check whether this holds.

The knowledge of amplitudes corresponding to inserting B-branes in only one patch, can be used to compute the whole of $|V\rangle$. Consider the two-point functions $\left\langle 0\left|\psi\left(x_{i}\right) \psi^{*}\left(x_{j}\right)\right| V\right\rangle$. For fermions in the same patch, we can write this as

$$
\left\langle 0\left|\psi\left(x_{i}\right) \psi^{*}\left(\widetilde{x}_{i}\right)\right| V\right\rangle=G\left(x_{i}, \widetilde{x}_{i}\right) \Psi\left(x_{i}\right) \Psi^{*}\left(\widetilde{x}_{i}\right)
$$

where $G\left(x_{i}, \widetilde{x}_{i}\right)$ is the Green's function of free-fermions on a quantum Riemann surface. Moreover, the knowledge of $\Psi\left(x_{i}\right)$ and $G\left(x_{i}, \widetilde{x}_{i}\right)$ at any one of the punctures allows us to compute, by "parallel transport", all other two-point functions $\left\langle 0\left|\psi\left(x_{j}\right) \psi^{*}\left(x_{k}\right)\right| V\right\rangle$ for $j, k \neq i$. Using (4.12)

$$
\psi\left(x_{j}\right)=\int d \widetilde{x}_{i} e^{-S\left(\widetilde{x}_{i}, x_{j}\right) / g_{s}} \psi\left(\widetilde{x}_{i}\right)
$$

we have

$$
\left\langle 0\left|\psi\left(x_{j}\right) \psi^{*}\left(x_{k}\right)\right| V\right\rangle=\int d x_{i}^{\prime \prime} \int d x_{i}^{\prime} e^{S\left(x_{i}^{\prime \prime}, x_{k}\right) / g_{s}-S\left(x_{i}^{\prime}, x_{j}\right) / g_{s}} \Psi\left(x_{i}^{\prime}\right) \Psi^{*}\left(x_{i}^{\prime \prime}\right) G\left(x_{i}^{\prime}, x_{i}^{\prime \prime}\right) .
$$

Moreover, it follows that it suffices to check the bilinearity in one patch only 5 .

5 This is because the defining property of $|V\rangle$ is that the correlation functions of fermions are given by determinants of $G\left(x_{i}, x_{j}\right)$ and this is preserved by canonical transformations. 


\subsection{Non-compact brane probes and Kontsevich-like matrix models}

As discussed in the previous section we can view the closed string deformation of geometry, in the patch with local coordinate $x_{j}$, parameterized by the couplings $t_{n}^{i}$ as being induced by branes at $x^{i}=x_{j}^{i}, j=1, \ldots N$. In terms of string theory description what this means is that if we denote the partition function of closed strings by $Z_{\text {closed }}\left(t_{n}^{i}\right)$ and that of the open string sector by $Z_{\text {open }}$ then

$$
Z_{\text {closed }}(0) Z_{\text {open }}\left(x_{j}^{i}\right)=Z_{\text {closed }}\left(t_{n}^{i}\right),
$$

where the $t_{n}^{i}$ are determined from the geometry of the branes located at $x_{i}^{j}$ in each asymptotic geometry given by $x^{i} \rightarrow \infty$. These parameters are related to each other by

$$
t_{n}^{i}=\frac{g_{s}}{n} \sum_{j}\left(x_{j}^{i}\right)^{-n} .
$$

This in particular means that the $t_{n}^{i}$ dependence of $Z_{\text {closed }}\left(t_{n}^{i}\right)$ can be computed by integrating the field theory living on the brane, which is given by $Z_{\text {open }}\left(x_{j}^{i}\right)$. This suggests that another way to compute the closed string amplitudes is to use the open string sector which deforms it.

The action for the open string sector has already been studied [14]. Let us, for simplicity first write it for one brane probe, located at $x=x_{1}$ which is defined near the asymptotic region $x \rightarrow \infty$. Then the tree level action, as a function of varying the position of the brane to a generic point $x$ is computed by considering the disc diagram in the B-model and is given by integrating the 1 -form $\lambda=x d p$

$$
S(p)=\frac{1}{g_{s}}\left(\int^{p} x\left(p^{\prime}\right) d p^{\prime}-x_{1} p\right)=\frac{1}{g_{s}}\left[W(p)-x_{1} p\right],
$$

where $x(p)$ is found by solving $H(p, x)$ and

$$
W(p)=\int^{p} x\left(p^{\prime}\right) d p^{\prime}
$$

Note that the extremum of the action gives the classical solution

$$
d S / d p=0 \rightarrow x(p)=x_{1} .
$$

which is consistent with the classical position of the brane. If we have instead of one brane, $N$ branes at positions

$$
\Lambda=\left(x_{1}, \ldots, x_{N}\right),
$$


we can write a matrix model version of the above action where we treat $p$ as an $N \times N$ matrix, which we denote by $P$ and $\Lambda$ as a diagonal $N \times N$ matrix. We thus have a matrix action

$$
S(P)=\frac{1}{g_{s}} \operatorname{Tr}[W(P)-\Lambda P],
$$

In general there can be quantum corrections to this classical action, which can include multi-trace contributions. In some cases there is no quantum correction in which case we get a very simple matrix model description of the closed string amplitude:

$$
Z_{\text {closed }}\left(t_{n}\right)=\int D P \exp \left[\frac{1}{g_{s}} \operatorname{Tr}(W(P)-\Lambda P)\right]
$$

This is a Kontsevich-like matrix model, where the source term has the data about the classical position of the branes and satisfies

$$
t_{n}=\frac{g_{s}}{n} \operatorname{Tr} \Lambda^{-n}
$$

This is assuming that $\partial \phi$ has integral expansion powers in $x$, as in (4.9). However, in some examples (such as the KdV hierarchy) we may have for geometric reasons a monodromy in $\partial \phi$ as $x \rightarrow e^{2 \pi i} x$, in which case the above expansion modes $n$ will be fractional. Later in this paper we will show that the original example of Kontsevich arises from exactly such a picture. Note that we do not need $N$ to have a large value for this relation to be true. It is true for any $N$. Of course for finite $N$ we only get an $N$-dimensional subspace of all the allowed values of $t_{n}$ 's.

We can in fact state the above from a slightly different perspective, but in a way which includes the quantum corrections. Namely, consider $N$ B-branes in some patch where we have, say $\left(x_{i}, p_{i}\right)$ as coordinates, and relate this to B-branes in different coordinates $\left(x_{j}, p_{j}\right)$, related to the original ones by a symplectic transformation generated by $S\left(x_{i}, x_{j}\right)$ which is quadratic in the variables.

$$
S\left(x_{i}, x_{j}\right)=\left(-\frac{d}{2} x_{i}^{2}+x_{i} x_{j}-\frac{a}{2} x_{j}^{2}\right) / c .
$$

We can then write the amplitude

$$
\left\langle N\left|\psi\left(x_{j, 1}\right) \ldots \psi\left(x_{j, N}\right)\right| V\right\rangle=\prod_{n<m}\left(x_{j, n}-x_{j, m}\right) e^{\mathcal{F}\left(x_{j}\right)}
$$

in terms of

$$
\left\langle N\left|\psi\left(x_{i, 1}\right) \ldots \psi\left(x_{i, N}\right)\right| V\right\rangle
$$




$$
\left\langle N\left|\psi\left(x_{j, 1}\right) \ldots \psi\left(x_{j, N}\right)\right| V\right\rangle=\int \prod_{n=1}^{N} d x_{i, n} e^{-\sum_{n=1}^{N} S\left(x_{i, n}, x_{j, n}\right)}\left\langle N\left|\psi\left(x_{i, 1}\right) \ldots \psi\left(x_{i, N}\right)\right| V\right\rangle
$$

But this is just an eigenvalue representation of the matrix integral

$$
Z\left(X_{j}\right)=\int D X_{i} e^{-\operatorname{Tr} S\left(X_{i}, X_{j}\right)} Z\left(X_{i}\right)
$$

where $Z\left(X_{i}\right)=e^{\mathcal{F}\left(X_{i}\right)}$ expressed in terms of $\operatorname{Tr} X_{i}^{n}=\sum_{m} x_{i, m}^{n}$. We have used here the well known expression for the Itzykson-Zuber integral [24]. As we discussed above, in some cases, such as the $(m, 1)$ minimal models, one can find variables $\left(p_{i}, x_{i}\right)$ such that $Z\left(X_{i}\right)$ is simple and the above is an effective way to evaluate the amplitudes corresponding to other patches.

\subsection{Compact and non-compact branes}

We can also consider both non-compact branes and compact branes at the same time. This will give rise not only to a transition in the compact parts of the geometry but influence the asymptotic geometry of the Calabi-Yau as well. As for the action for the branes this means that in addition to the matrix model action $W(\Phi) / g_{s}$ describing the compact branes, and the corresponding contribution of the non-compact brane we also have to integrate out the open strings stretched between the compact and the non-compact branes. This is the mirror situation to that considered in [25] which leads to determinant of the mass of the open strings stretched between them. In the above geometry (4.4), if the non-compact B-brane is at $x$ this leads to the mass

$$
M=x-\Phi,
$$

which thus leads to modification of $Z$ by the inclusion of $\operatorname{det}(x-\Phi)$, or by the changing of the action by

$$
\delta S=\operatorname{Tr} \log (x-\Phi)
$$

Note that this is up to a factor of 2 the same as adding one extra eigenvalue to $\Phi$ and freezing it at $x$. 


\subsection{Fermion number flux and higher genera}

Let us consider the Riemann surface $\Sigma: H(p, x)=0$ of genus $g$. If $g>0$, we can obtain the amplitudes by sewing of $g=0$ case, as is familiar in the operator formulation of Riemann surfaces. However, there is one subtlety with this sewing prescription: we can ask what is the fermion number (the momentum of the $\phi$ field) going around the loops (say along the $A$-cycles) of the Riemann surface. In fact we have to have a particular treatment of the momenta around loops to get a unique answer for our amplitudes in such cases. This issue does not arise when $g=0$, and in such cases any fermion operators we insert at the boundaries can be absorbed by shifting the vacuum fermion number at the corresponding Hilbert space, as discussed before.

There are two natural choices one can make: One is to set the momenta in the loops to be zero. This case turns out to be the natural choice for computation of the B-model amplitudes. However, it is not so natural from the viewpoint of free fermions: If we wish to retain a free fermionic description for higher genus as well, it is natural not to fix the fermion number around the loops. We introduce $g$ new variables $\theta_{1}, \ldots, \theta_{g}$ and consider

$$
Z\left(\theta_{i}\right)=\sum_{N_{i}} Z_{N_{1}, \ldots, N_{g}} \exp \left(i N_{i} \theta_{i}\right)
$$

This has the advantage that the amplitudes can still be constructed using free fermions (i.e. the vertex is still exponential of bilinear fermion terms instead of sum of such exponentials). Of course we can obtain the answer for the case where we have fixed the momenta to be zero from this expression by considering

$$
Z_{N_{i}=0}=\int \prod_{i=1}^{g} d \theta_{i} Z\left(\theta_{i}\right)
$$

It may appear that there is more information in $Z\left(\theta_{i}\right)$ than in $Z_{N_{i}=0}$. However this is not the case, because there is some redundancy in $Z_{N_{i}}$. This can be seen as follows: Consider adding a brane/antibrane at a point on the Riemann surface $\Sigma$ and move the brane around a non-trivial cycle, say $B_{i}$ and bring it back to annihilate the anti-brane. In doing so we have changed the fermion number flux through the $A_{i}$ cycle by one unit. This can be equivalently viewed as changing the moduli of the Calabi-Yau (this was also observed in a related context in [26]), and in particular the Riemann surface $\Sigma$. Note that 
as the brane traverses along the $B_{i}$ cycle on the Riemann surface, it spans a 3 -cycle $C$ in the Calabi-Yau. According to (4.1) this changes the integral of $\Omega$ along three-cycle $C^{\prime}$ by

$$
\Delta \int_{C^{\prime}} \Omega=\#\left(C \cap C^{\prime}\right) g_{s}
$$

Let us denote the moduli of the Calabi-Yau by $\int_{C_{i}} \Omega=S_{i}$ and let the intersection number of $C_{i}$ and $C$ be $\#\left(C_{i} \cap C\right)=m_{i}$. Then we have

$$
Z_{N_{i}}\left(S_{i}\right)=Z_{N_{i}=0}\left(S_{i}+N_{i} m_{i} g_{s}\right)
$$

and

$$
Z\left(\theta_{i}\right)=\sum_{N_{i}} \exp \left(i N_{i} \theta_{i}\right) Z_{N_{i}=0}\left(S_{i}+N_{i} m_{i} g_{s}\right)
$$

\section{Examples}

In this section we consider four classes of examples to illustrate the ideas developed in the previous sections.

In the first class of examples we consider CY geometries of the form

$$
H(p, x)=\sum_{i=0}^{r} p^{i} f_{i}(x)
$$

where $f_{i}(x)$ is a power series in $x$ (possibly a finite polynomial). These geometries can also be viewed as being obtained from matrix models associated with $A_{r-1}$ quiver diagrams, with $r-1$ adjoint matrices with potentials $W\left(\Phi_{i}\right)$ interacting with bifundamental fields. The case $r=1$ corresponds to the KP hierarchy. We also discuss the relation of these theories to $(r, *)$ minimal models. The case $r=1$ can be viewed as taking a particular limit of these geometries. We show in the case of $(1, p)$ models how the brane probe picture gives rise to the Kontsevich-like models (the case $p=2$ is the original Kontsevich model). These are also equivalent to the $p$-th, $\mathcal{N}=2$ minimal model coupled to topological gravity [27,23]. We also explain the meaning of Virasoro (or more generally $\mathcal{W}$-constraints) for these models, as simply encoding the fact that Calabi-Yau is non-singular.

In the second example we consider CY geometry corresponding to the conifold $H(p, x)=x p-\mu$ and its deformations. As has been noted in [8] this is equivalent to topological string for $c=1$ bosonic string at self-dual radius. We show how one can interpret the results of [12] from the perspective of the present paper, as well as how the Toda hierarchy arises from the Calabi-Yau geometry. 
In the third example we consider the mirror of A-model topological string on $\mathbf{P}^{1}$ coupled to topological gravity. This is a model in which $H(p, x)=x+e^{p}+q e^{-p}$ where $x \in \mathbf{C}$ and $p \in \mathbf{C}^{*}$.

In the fourth example we consider the mirror of A-model topological string on $\mathbf{C}^{3}$. This is the case where $H(p, x)=e^{x}+e^{p}-1$. We recover the topological vertex [9] from the perspective of this paper from various approaches. We also give the generalization of this to B-model mirrors of arbitrary toric Calabi-Yau manifolds.

\subsection{Example 1: Matrix models and the KP hierarchy}

It might be useful to first consider the case of geometries that naturally appear in matrix models and their double scaling limit. Let $W(x)$ be a polynomial of degree $n+1$. Consider, as in [4], $N$ compact B-branes in

$$
z w+p^{2}-W^{\prime}(x)^{2}=0
$$

where the B-branes are wrapping $n \mathbf{P}^{\mathbf{1}^{\prime}} s$ at $W(x)^{\prime}=0$, as we discussed in section 4.1. The open topological string is described by the saddle-point expansion of the $N \times N$ matrix integral

$$
Z=\frac{1}{\operatorname{vol} U(N)} \int d \Phi_{N \times N} \cdot \exp \left[\frac{1}{g_{s}} \operatorname{Tr} W(\Phi)\right] .
$$

In the 't Hooft limit $N \rightarrow \infty, g_{s} \rightarrow 0$ with $g_{s} N$ fixed, this describes closed topological strings in the Calabi-Yau geometry

$$
z w+p^{2}-W^{\prime}(x)^{2}-f(x)=0
$$

Here

$$
W(x)=\sum_{i=0}^{n+1} t_{i} x^{i}
$$

is a given polynomial, and $f(x)$ is a polynomial of degree $n$ - the quantum correction that is determined by the saddle-point around which the expansion is performed. More precisely, $f(x)$ is encoded by the distributions $N_{i}$ of the eigenvalues of $\phi$ among the $d$ critical points of $W$ through the 't Hooft couplings $s_{i}=g_{s} N_{i}$.

The conjugate variable $p(x)$ appears naturally as (the expectation value of) the resolvent of the matrix model

$$
p(x)=W^{\prime}(x)+2 g_{s} \operatorname{Tr} \frac{1}{x-\Phi} .
$$


The corresponding meromorphic one-form can be written as

$$
\lambda=p(x) d x=d \phi(x)
$$

with

$$
\phi(x)=W(x)+2 g_{s} \operatorname{Tr} \log (x-\Phi) .
$$

This collective fields has an interpretation as the effective action of a single extra eigenvalue in the background of the $N$ dynamical eigenvalues of the matrix $\Phi$. Identifying the onepoint functions in the matrix model with derivatives of the partition function (in the genus zero limit, with $\log Z=\sum_{g \geq 0} g_{s}^{2 g-2} \mathcal{F}_{g}$ ) gives the relation

$$
\left\langle\operatorname{Tr} X^{n}\right\rangle=g_{s} \frac{\partial \mathcal{F}_{0}}{\partial t_{n}}
$$

Using this fact we can write the mode expansion of $\partial \phi$ in the, by now familiar, form

$$
\partial \phi(x)=\sum_{n>0} n t_{n} x^{n-1}+2 g_{s}^{2} \sum_{n>0} \frac{\partial \mathcal{F}_{0}}{\partial t_{n}} x^{-n-1}
$$

The hyperelliptic curve

$$
H(p, x)=p^{2}-W^{\prime}(x)^{2}-f(x)=0
$$

that gives the relation between $x$ and $p$ emerges as the large $N$ limit of the loop equations of the matrix model.

Note that this curve has two set of moduli. The unnormalizable ones correspond to deformations of the couplings $t_{k}$ in the matrix model potential. The corresponding deformations of the meromorphic one-form $\delta \lambda$ are given by Abelian differentials of the second kind, that behave as $\sim x^{n+k} d x$ in the limit $x \rightarrow \infty$. The couplings $s_{k}$ induce normalizable deformations $\delta \lambda \sim x^{k} d x$ that are given by Abelian differentials of the first kind and correspond to variations of the quantum correction $f(x)$. We will focus our discussion here on the unnormalizable deformation $t_{k}$ that are visible at $x \rightarrow \infty$.

In the standard interpretation of the (genus zero) loop equations one considers the general deformation

$$
p(x)=p_{c l}(x)+\partial \phi(x)
$$


where $\partial \phi$ has the mode expansion (5.2). This includes terms $x^{-n}$ that have poles in the interior $x \rightarrow 0$. However, the deformation should be such that the corresponding hyperelliptic curve stays smooth away from infinity. Therefore the stress tensor

$$
T(x)=\partial \phi^{2}=p^{2}=\sum_{n \in Z} L_{n} x^{-n-2}
$$

should be regular in the interior. In particular, the modes $L_{n}$ with $n \geq-1$ should vanish. These are the famous Virasoro constraints.

In the full quantum theory these relations still hold, but now are implemented as operator relations. That is, one writes the partition function as a wave function in a coherent state basis

$$
Z=\langle t \mid V\rangle
$$

and requires

$$
L_{n}|V\rangle=0, \quad n \geq-1 .
$$

Now $L_{n}$ are the modes of the quantum stress tensor.

By generalizing to matrix chains, more precisely quiver matrix models of type $A_{r-1}$ $28,29,5$, one obtains curves that are $r$-folded covers of the eigenvalue $x$-plane given by a schematic equation of the form

$$
p^{r}+\ldots+F(x)=0
$$

In that case the corresponding matrix model partition function is not determined by the quadratic Virasoro constraints, but by $W$-generators of order $r$ in the collective field $p=$ $\partial \phi(x)$.

\subsection{Branes in the Gaussian matrix model}

In the light of the discussion we have had in the previous sections, we now revisit the matrix model and interpret it in terms of branes. Quite generally, putting a brane in the matrix model is implemented by shifting the couplings in the superpotential

$$
t_{k} \rightarrow t_{k}+\frac{g_{s}}{k} x^{-k}
$$

The brane creation operator is given by

$$
\psi(x)=e^{\phi(x) / g_{s}}=e^{W(x) / g_{s}} \operatorname{det}(x-\Phi)
$$


where we recognize the classical and the quantum contribution. Partition functions of branes are therefore represented as correlation functions of characteristic polynomials in the matrix model. For example, a $M$-point function is given by

$$
\left\langle M\left|\psi_{q u}\left(x_{1}\right) \cdots \psi_{q u}\left(x_{M}\right)\right| V\right\rangle=\left\langle\operatorname{det}\left(x_{1}-\Phi\right) \cdots \operatorname{det}\left(x_{M}-\Phi\right)\right\rangle
$$

where $\langle\cdots\rangle$ denotes a normalized expectation value in the matrix model.

Using the methods of orthogonal polynomials one can easily evaluate such correlators. Let $\left\{P_{i}(x)\right\}_{i \geq 0}$ be a basis of orthogonal polynomials for the matrix model (5.1), normalized such that $P_{i}(x)=x^{i}+\ldots$, and let

$$
\Psi_{i}(x)=P_{i}(x) e^{W(x) / 2 g_{s}}
$$

be the corresponding "wave functions." Then one expresses the brane $n$-point function as an $n \times n$ Slater determinant [30]

$$
\left\langle\operatorname{det}\left(x_{1}-\Phi\right) \cdots \operatorname{det}\left(x_{M}-\Phi\right)\right\rangle=\frac{\operatorname{det} P_{N+j-1}\left(x_{i}\right)}{\Delta(x)}
$$

with Vandermonde determinant

$$
\Delta(x)=\operatorname{det} x_{i}^{j-1}
$$

Adding the classical contribution we get

$$
\left\langle\psi\left(x_{1}\right) \cdots \psi\left(x_{M}\right)\right\rangle=\frac{\operatorname{det} \Psi_{N+j-1}\left(x_{i}\right)}{\Delta(x)} .
$$

In particular for the one-point function we have

$$
\langle\psi(x)\rangle=\Psi_{N}(x)
$$

This relation is particularly illuminating in the simple case of the Gaussian model

$$
Z=\frac{1}{\operatorname{vol} U(N)} \int d \Phi \cdot \exp \left[-\frac{1}{2 g_{s}} \operatorname{Tr} \Phi^{2}\right]
$$

This corresponds to the deformed geometry

$$
H(p, x)=p^{2}+x^{2}-\mu=0
$$


with $\mu=g_{s} N$. In this case (and only in this case) the orthogonal polynomials are actual eigenfunction of a Schrödinger operator. The polynomials $P_{i}(x)$ are Hermite polynomials, and therefore the corresponding wave functions satisfy

$$
\left(-g_{s}^{2} \frac{\partial^{2}}{\partial x^{2}}+x^{2}\right) \Psi_{k}(x)=g_{s}\left(k+\frac{1}{2}\right) \Psi_{k}(x) .
$$

Note that in this case, where we recall that $\mu=g_{s} N$, we can verify that the one-point function $\langle\psi(x)\rangle=\Psi_{N}(x)$ indeed satisfies (up to the quantum shift $N \rightarrow N+\frac{1}{2}$ )

$$
H \Psi_{N}=0
$$

This relation is not as straightforward in the more general non-Gaussian matrix model. In that case the corresponding Riemann surface has genus $g>0$. Therefore there are nontrivial loop momenta or fluxes

$$
\frac{1}{2 \pi} \oint_{A_{i}} p d x=g_{s} N_{i}
$$

As we discussed before the existing of this "bulk" moduli makes the free fermion formulation less straightforward, since we now have to project on fixed loop charges going through the handles of the Riemann surface. This is directly related to the fact that the saddlepoint approximation of the matrix model is not captured by the method of orthogonal polynomials. It would be interesting to push this connection further.

Returning to the Gaussian matrix model, let us now derive the Kontsevich matrix model description of this. This corresponds to putting $M$ non-compact B-branes in the geometry

$$
z w=p(p-x)-\mu
$$

near $x \rightarrow \infty$ (where we changed variables apropriately). This, as explained in section 4.5. is described by the Kontsevich-type matrix model with action

$$
S=\operatorname{Tr}\left[\Lambda X-\int X(P) d P\right]
$$

where $x(p)$ solves $p(p-x)=\mu$. So we get the matrix model

$$
\int_{M \times M} D P \operatorname{det}(P)^{N} e^{\operatorname{Tr}\left[\Lambda P-P^{2} / 2\right] / g_{s}}
$$

where we have used that $\mu / g_{s}=N$. 
This also leads us to a physical interpretation of the claim 30] of the equivalence of a general 1-matrix model

$$
\int D X_{N \times N} e^{\left[\operatorname{Tr} X^{2} / 2+\sum_{n>2} t_{n} \operatorname{Tr} X^{n}\right] / g_{s}}
$$

with this particular Kontsevich-type matrix model (5.5).

Namely, as we have explained above, the matrix model in (5.6), for small $t_{n}$ is describing $N$ compact B-branes on the $\mathbf{P}^{1}$ in the conifold

$$
z w=p(p-x)
$$

and deformations by $t_{n}$ 's can alternatively be viewed in terms of turning on nonnormalizable deformations

$$
z w=p\left(p-W^{\prime}(x)\right)
$$

where

$$
W^{\prime}\left(x, t_{n}\right)=x-\sum_{n>2} t_{n} x^{n-1}
$$

or placing non-compact B-branes at $x=x_{i}, \sum_{i} x_{i}^{-n}=\operatorname{Tr} \Lambda^{n}$, as we discussed above in addition to the compact ones. The large $N$ dual description of this can, on the one hand, be viewed as closed string theory on

$$
z w=p\left(p-W^{\prime}(x)\right)-\mu W^{\prime}(x) / x
$$

where $\mu=N g_{s}$ and the deformation of the geometry corresponds to growing a single $S^{3}$ at $x=0$, corresponding to $t_{n}$ 's being small deformations around the deformed conifold background $z w=y(y-x)-\mu$. On the other hand, it is given by placing B-branes at fixed $x$ in (5.4). Thus we have reproduced the result shown in [30 using the methods of orthogonal polynomials.

\subsection{Double scaling limits}

The above discussion is perhaps a bit confusing, since (for the one matrix model) the corresponding hyperelliptic Riemann surface has two asymptotic ends, distinguished by the sign of $p(x) \sim \pm x^{d}$ in the limit $x \rightarrow \infty$, or equivalently by the choice of the sheet of the two-fold cover. A priori one can have independent deformations at both end, as we 
will see in a moment when we consider the $c=1$ string and the Toda hierarchy. Above we treated both ends symmetrically.

This situation simplifies if we take a double scaling limit [31, 32,33]. Geometrically this corresponds to considering the local geometry where we send $2 k+1$ of the original $2 d$ branch points close together and zoom in at that region. (Equivalently, one can send the remaining branch points to infinity.) In that case the Riemann surface takes the form

$$
p^{2}+x^{2 k+1}+\ldots=0
$$

where the ellipses indicate terms of lower order in $x$. In such a geometry there is only one asymptotic region $x \rightarrow \infty$, where the variable $x$ is a good coordinate.

This particular double scaling limit of the matrix model is well-known to correspond to a non-critical bosonic string theory based on the $(2,2 k+1)$ minimal model coupled to two-dimensional gravity [27,34]. In particular, in the simplest case where we zoom in on a single branch point, we have the geometry

$$
p^{2}+x=0
$$

which corresponds to the $(2,1)$ model or pure topological gravity.

In this case we can introduce again the Kodaira-Spencer field $\phi(x)$, which now has a twisted mode expansion, because the global geometry enforces that at infinity

$$
p\left(e^{2 \pi i} x\right)=-p(x)
$$

If we expand this twisted boson as

$$
p(x)=\partial \phi(x)=\sum_{n \geq 0} n t_{n} x^{n-\frac{1}{2}}+2 g_{s}^{2} \sum_{n \geq 0} \frac{\partial}{\partial t_{n}} x^{-n-\frac{3}{2}}
$$

we make contact with the usual description of topological gravity, where the coupling $t_{n}$ couples to the descendant $\tau_{n}$ of the identity operator.

Besides the twisting, we have precisely the same argument that fixes the solution of this deformation problem. The function $p(x)^{2}$ should again be smooth in the interior of the $x$-plane. Apart from $x=\infty$ there should be no extra poles, i.e. no new asymptotic ends. This then gives again the Virasoro constraints

$$
L_{n}|V\rangle=0
$$

It is well-known that these constraints are equivalent to statement that the partition function $Z$ is a tau function of the $\mathrm{KdV}$ hierarchy that satisfies the so-called string equation $33,34,35$. 


\subsection{The $(m, 1)$ minimal models}

One can also consider the double-scaled quiver matrix model that gives rise to the $(r, s)$ minimal model coupled to gravity. This leads to the effective geometry

$$
p^{r}+x^{s}+\ldots=0 .
$$

In particular, consider $(m, 1)$ topological minimal models. The corresponding Riemann surface is given by

$$
p^{m}+x=0
$$

before turning on the deformations. This is known to be governed by a generalized KdV hierarchy with Lax operator, $L(t=0)=L_{0}$ at zero times, given by

$$
L_{0}=D^{m}+x
$$

where $D=\frac{\partial}{\partial x}$. Notice that the Lax operator is the equation of the Riemann surface with $D=p$. Moreover, the one-point function, corresponding to inserting a non-compact B-brane at fixed value of $x$

$$
\Psi(x, t)=\langle t|\psi(x)| V\rangle,
$$

is known in the integrable systems literature as the Baker-Akhiezer function, and $\Psi(x)=$ $\Psi(x, 0)$ is annihilated by $L_{0}$

$$
L_{0} \Psi(x)=0
$$

(We can relax the eigenvalue to be arbitrary, but this can be reabsorbed by shifting $x$ ). Turning on the times of the KdV hierarchy can thus be identified with placing non-compact B-branes at fixed values of $x$. In terms of the $(m, 1)$ minimal model, this is computing the topological string partition function deformed by the gravitational descendants.

We now want to derive the Kontsevich matrix model which also describes this. To do so, consider first a B-brane in the $p$ patch. In this case, we have

$$
x=\partial \phi(p)=p^{m}+\sum_{n>0} n t^{n} p^{n-1}+\frac{\partial \mathcal{F}}{\partial t_{n}} p^{-n-1}
$$

in terms of the classical piece and the quantum one. In this case, there is no quantum correction, $\mathcal{F}=0$ and the classical result is exact. This is because the Ward identity

$$
\oint \psi^{*}(p) p^{n} \psi(p)|V\rangle=0
$$


for $n>0$ implies that $\partial \mathcal{F} / \partial t_{n}=0$ for any $n$ which together with the Riemann surface equation as an initial condition, implies that $\mathcal{F}$ is zero all-together. We see that

$$
\Psi(p)=\langle\psi(p)\rangle=\exp \left[\frac{1}{g_{s}} \frac{p^{m+1}}{m+1}\right]
$$

where $\frac{p^{m+1}}{m+1}$ agrees with the classical action $\int x(p) d p$. Moreover, we have that

$$
H(p, x) \Psi(p)=\left(p^{m}+x\right) \Psi(p)=0
$$

with $x=-g_{s} \frac{\partial}{\partial p}$.

To describe the topological $(p, 1)$ minimal model deformed by various observables, as we discussed above, we need to consider non-compact B-branes at fixed values of $x$. We can do this by relating them to B-branes in the $p$-variable, which are simple, and considering symplectic transformations. The canonical transformation that relates the two descriptions is just the $S$ transformation, i.e., the Fourier transform

$$
\Psi(x)=\int d p e^{-x p / g_{s}} \Psi(p)=\int d p \exp \left[\left(-x p+\frac{p^{m+1}}{m+1}\right) / g_{s}\right]
$$

We can now see how the Kontsevich matrix model arises, namely, by putting $N$ B-branes at fixed value of $x$. This leads, as discussed before, to a Kontsevich-like matrix model given by (4.16) with action

$$
S=\frac{1}{g_{s}} \operatorname{Tr}\left[-\Lambda P+\int X(P) d P\right]=\frac{1}{g_{s}} \operatorname{Tr}\left[-\Lambda P+\frac{1}{m+1} P^{m+1}\right]
$$

The matrix $\Lambda$ fixes the classical $x$ positions of the branes. The couplings

$$
t_{k}=\frac{g_{s}}{k+\frac{1}{m}} \operatorname{Tr} \Lambda^{-k-\frac{1}{m}}=\frac{g_{s}}{k+\frac{1}{m}} \sum_{i} x_{i}^{-k-\frac{1}{m}}
$$

encode the deformed geometry by

$$
p=x^{\frac{1}{m}}+\sum_{k=0}^{\infty} t_{k} x^{k+\frac{1}{m}}+O(1 / x)
$$

where we have taken the asymptotic monodromy of $p$ into account.

This model is equivalent to bosonic $(m, 1)$ minimal model coupled to gravity. On the other hand it is also known that this is equivalent to the B-model topological string 
coupled to minimal $\mathcal{N}=2$ model [36, 37], which is given by LG theory with one chiral field $P$ with superpotential 38

$$
W(P)=P^{m}
$$

From the above relation we conclude that if we consider coupling of the B-model theory with superpotential $W(P)$ to topological strings then this is equivalent to considering B-model topological strings on the Calabi-Yau 3-fold

$$
z w-x-W(P)=0
$$

This may also be possible to explain more directly by showing that the worldsheet theory of Calabi-Yau and that of the theory with superpotential $W(P)$ coupled to topological gravity are the same. In fact there is some evidence that this is not too difficult. It has been shown in [39] that the conformal theory of strings propagating on the $A_{m-1}$ singularity of $K 3$

$$
z w-P^{m}=\mu
$$

is given (up to a $\mathbf{Z}_{m}$ orbifold) by the tensor product of the $N=2$ SCFT with superpotential $P^{m}$ times a Liouville system. The geometry we are considering here is a simple extension of this where $\mu$ is varying over an extra dimension parameterized by $x, \mu(x)=x$. It would be interesting to complete this identification. We will see another example of this when we consider below topological strings on $\mathbf{P}^{1}$.

\subsection{Example 2: $c=1$ strings and the Toda hierarchy}

The $c=1$ non-critical string compactified on the self-dual radius $R=1$ is well-known to be equivalent to B-model topological strings on the conifold. In this case we are dealing with Hamiltonian

$$
H=p^{2}-x^{2}-\mu \text {. }
$$

We will change variables to

$$
x, y=p \pm x
$$

so that the level set is given by the curve

$$
x y=\mu \text {. }
$$






Fig. 1: The geometry relevant to the $c=1$ string at selfdual radius is the complex curve $x y=\mu$.

Note that this curve has two asymptotic regions (see fig. 1) described by $x \rightarrow \infty$ and $y \rightarrow \infty$. These regions correspond to the incoming and outgoing excitations in the usual $S$-matrix formulation of the $c=1$ matrix model.

At tree-level this model describes the Kodaira-Spencer theory of the curve $x y=\mu$. Here one parameterizes these deformations as Laurent expansions of $y(x)$ in the $x$-patch and $x(y)$ in the $y$-patch by

$$
y=-\partial_{x} \phi(x), \quad x=\partial_{y} \widetilde{\phi}(y) .
$$

Here the $\phi$ 's have a classical piece $\phi_{c l}(x), \tilde{\phi}_{c l}(y)$, corresponding to the one-form on the Riemann surface, and a quantum piece, corresponding to the Kodaira-Spencer field $\phi_{q u}(x), \widetilde{\phi}_{q u}(y)$ in the two patches. The classical contributions read

$$
\partial_{x} \phi_{c l}(x)=-\frac{\mu}{x}, \quad \partial_{y} \widetilde{\phi}_{c l}(y)=\frac{\mu}{y},
$$

whereas the quantum parts have mode expansions

$$
\begin{aligned}
& \partial_{x} \phi_{q u}(x)=\sum_{n>0} n t_{n} x^{n-1}+\sum_{n>0} \frac{\partial}{\partial t_{n}} x^{-n+1}, \\
& \partial_{y} \widetilde{\phi}_{q u}(y)=\sum_{n>0} n \widetilde{t}_{n} y^{n-1}+\sum_{n>0} \frac{\partial}{\partial \widetilde{t}_{n}} y^{-n+1} .
\end{aligned}
$$

The coefficients $t_{n}, \widetilde{t}_{n}$ of the unnormalizable modes (in the limit of $x, y \rightarrow \infty$ ) should be seen as coupling constants, while the coefficients of normalizable modes have an interpretation 
as one-point functions of the dual operators $\partial_{n}$. Note that the couplings and one-point functions can be written, in the classical limit, as periods $\left(\oint \equiv \frac{1}{2 \pi i} \int\right)$, e.g.

$$
\begin{aligned}
t_{n} & =\frac{1}{n} \oint_{x \rightarrow \infty} y x^{-n} d x \\
\frac{\partial F_{0}}{\partial t_{n}} & =\oint_{x \rightarrow \infty} y x^{n} d x
\end{aligned}
$$

With this substitution the consistency of the equations

$$
y=\partial \phi(x)=f(x), \quad x=\partial \widetilde{\phi}(y)=f^{-1}(y),
$$

is equivalent to the fact that the function $\mathcal{F}_{0}\left(t_{n}, \widetilde{t}_{n}\right)$ solves the dispersionless limit of the two-Toda hierarchy.

One can also easily solve this model in terms of Ward identities, at least at treelevel. Note that in this case the curve has topology $\mathbf{C}^{*}$ or a twice punctured sphere. Let $P=\{x=\infty\}$ and $Q=\{y=\infty\}=\{x=0\}$ denote the north and south pole respectively. Now consider the action of the $\mathcal{W}$-current $y=\partial \phi$. More precisely, consider the charge $\oint_{P} x^{n} y$. If we move the contour to the south pole $Q$ we obtain the identity

$$
\oint_{P} x^{n} y=\oint_{Q} \frac{1}{n+1} x^{n+1} y .
$$

In other words, we have

$$
\frac{\partial \mathcal{F}}{\partial t_{n}}=\oint_{Q} \frac{1}{n+1}(\partial \widetilde{\phi}(y))^{n+1} .
$$

Note that the bosons $\phi$ and $\widetilde{\phi}$, in the language of section 3 are related by the $S$ transformation that acts on the canonically conjugated variables $x$ and $y$ as

$$
S:(x, y) \rightarrow(y,-x)
$$

Therefore the fields $\phi(x)$ and $\widetilde{\phi}(y)$ are (semi-classically) related by a Legendre transform. This is highly non-linear transformation, as the above Ward identity clearly shows - the "in-coming" modes of $\partial \phi$ are polynomials in the "out-going" modes of $\partial \widetilde{\phi}$.

In accord with our general philosophy, the corresponding quantum relations are most natural written in terms of branes, which fermionize the boson fields in the usual fashion

$$
\psi=e^{\phi / g_{s}}, \quad \widetilde{\psi}=e^{\widetilde{\phi} / g_{s}} .
$$


The fermions of the two patches are related by Fourier transform

$$
\widetilde{\psi}(y)=S \psi(y)=\frac{1}{\sqrt{2 \pi}} \int d x e^{-x y / g_{s}} \psi(x) .
$$

In terms of the action of the $S$ matrix on the second-quantized fermion Fock space, the partition function can be written as

$$
Z(t, \widetilde{t})=\langle t|S| \widetilde{t}\rangle
$$

The quantum parts of the fermions, defined by $\psi=e^{\phi_{c l} / g_{s}} \psi_{q u}$ have mode expansions

$$
\psi_{q u}(x)=\sum_{p \in \mathbf{Z}+\frac{1}{2}} \psi_{p} x^{-p-\frac{1}{2}}, \quad \psi_{q u}^{*}(x)=\sum_{p \in \mathbf{Z}+\frac{1}{2}} \psi_{p}^{*} x^{-p-\frac{1}{2}},
$$

with canonical anti-commutation relations $\left\{\psi_{p}, \psi_{q}^{*}\right\}=\delta_{p+q, 0}$.

In terms of the fermions, we get quantum analogue of (5.9). Namely the operator $\oint_{P} x^{n} \partial \phi(x)$ corresponds to the $\mathcal{W}_{1+\infty}$ generator that shifts

$$
(x, y) \rightarrow\left(x, y+n x^{n-1}\right)
$$

so we have

$$
\oint_{x \rightarrow \infty} \psi^{*}(x) x^{n} \psi(x)|V\rangle=\oint_{y \rightarrow \infty} \psi^{*}(y)\left(g_{s} \partial_{y}\right)^{n} \psi(y)|V\rangle .
$$

This Ward identity for the $c=1$ string amplitude at selfdual radius was derived in [12] in this form, where the starting point was the well-known statement that in terms of fermions, the $c=1$ string is free. Namely, the $c=1$ string scattering matrix which relates the incoming and the outgoing modes of the fermions consists just of a phase factor

$$
\widetilde{\psi}_{-n-\frac{1}{2}}=R_{n+\frac{1}{2}} \psi_{-n-\frac{1}{2}} .
$$

Here the reflection factor $R_{q}$, for general momentum $q$, is given by

$$
R_{q}=i\left(\frac{1+i e^{-\pi(\mu+i q)}}{1-i e^{-\pi(\mu+i q)}}\right)^{\frac{1}{2}}\left(\frac{\Gamma\left(\frac{1}{2}-i \mu+q\right)}{\Gamma\left(\frac{1}{2}+i \mu-q\right)}\right)^{\frac{1}{2}}
$$

In fact, we can derive the fermion scattering amplitude $R_{q}$ directly from (5.10) as follows.

If we turn on one set of times only, the partition function is trivial by momentum conservation, and $\mathcal{F}(t, 0)=0$. Consequently, in the $x$ patch, the fermions are free, and the one-point functions are exact at the classical level

$$
\Psi(x)=\langle 0|\psi(x)| V\rangle=x^{-\mu},
$$


corresponding to contribution of the classical piece $e^{\phi_{c l}}$, in $\phi(x)=e^{\phi_{c l}(x)} \psi_{q u}(x)$. Since fermions transform as wave functions, this further implies that the fermion scattering state $|V\rangle$ is of general form

$$
|V\rangle=\exp \left(\sum_{m, n \geq 0} a_{m n} \psi_{-m-\frac{1}{2}} \tilde{\psi}_{-n-\frac{1}{2}}^{*}+\tilde{a}_{m n} \tilde{\psi}_{-m-\frac{1}{2}} \psi_{-n-\frac{1}{2}}^{*}\right)|0\rangle
$$

and we will now show that $a_{m n}=R_{n+\frac{1}{2}} \delta_{m n}, \tilde{a}_{m n}=R_{n+\frac{1}{2}}^{*} \delta_{m n}$, which will reproduce the result we quoted.

Consider $\left\langle 0\left|\psi(y) \psi^{*}(x)\right| V\right\rangle$. On the one hand, this equals

$$
(x y)^{-\mu} \sum_{m, n \geq 0} a_{m, n} x^{-m-1} y^{-m-1} .
$$

On the other hand, we can compute this knowing the correlators in the $x$-patch alone. For this, note that similarly to what we had in the $x$ patch,

$$
\tilde{\Psi}(y)=\langle 0|\tilde{\psi}(y)| V\rangle=y^{\mu} .
$$

Moreover, since in the $x$-patch, we have

$$
\left\langle 0\left|\psi(\widetilde{x}) \psi^{*}(x)\right| V\right\rangle=\frac{\widetilde{x}^{-\mu} x^{\mu}}{x-\widetilde{x}}
$$

then using (5.10) it follows,

$$
\left\langle 0\left|\tilde{\psi}(y) \psi^{*}(x)\right| V\right\rangle=\frac{1}{\sqrt{2 \pi i}} \int d \widetilde{x} e^{\tilde{i x y}} \frac{\widetilde{x}^{-\mu} x^{\mu}}{x-\widetilde{x}} .
$$

which equals

$$
\int d \widetilde{x} e^{\tilde{i x y}} \frac{\widetilde{x}^{-\mu} x^{\mu}}{x-\widetilde{x}}=(x y)^{\mu} \sum_{n \geq 0}(x y)^{-n-1} \widehat{R}_{n+\frac{1}{2}}
$$

where

$$
\widehat{R}_{n+\frac{1}{2}}=\frac{1}{\sqrt{2 \pi i}} \oint d \widetilde{x} \widetilde{x}^{-\mu} \widetilde{x}^{n} e^{i \widetilde{x}}
$$

Similarly, one finds that $\tilde{a}_{m n}=\widehat{R}_{n+\frac{1}{2}}^{*} \delta_{m n}$. It is easy to show that (see appendix A)

$$
\widehat{R}_{n+\frac{1}{2}}=R_{n+\frac{1}{2}}
$$

up to a constant multiple, and correspondingly

$$
|V\rangle=\exp \left(\sum_{n \geq 0} \widehat{R}_{n+\frac{1}{2}} \psi_{-n-\frac{1}{2}} \tilde{\psi}_{-n-\frac{1}{2}}^{*}+\widehat{R}_{n+\frac{1}{2}}^{*} \tilde{\psi}_{-n-\frac{1}{2}} \psi_{-n-\frac{1}{2}}^{*}\right)|0\rangle .
$$

When the times are turned on, the curve $x y=\mu$ gets deformed. The precise form of the deformation can be derived from the dispersionless solution by using that $x y=y \partial_{y} \widetilde{\phi}(y)=$ $-x \partial_{x} \phi(x)$. For example, if we turn on $t_{1}, \widetilde{t}_{1}$, the curve gets deformed to $\left(x-\widetilde{t}_{1}\right)\left(y+t_{1}\right)=\mu$. A detailed description of the deformed curve using the Toda hierarchy can be found for example in [40]. 


\subsection{Kontsevich-like model for $c=1$}

It has been known that there is a Kontsevich-like model also for $c=1$ string at selfdual radius [12,41]. We will now rederive this from the perspective of the present paper. Let us consider turning on only the couplings $t_{n}$, and set $\widetilde{t}_{n}=0$ momentarily. Then all the amplitudes are trivial, because of momentum conservation. Thus we obtain a simple deformation of the geometry

$$
x y-\mu=0 \longrightarrow x y+\sum_{n>0} n t_{n} x^{n}-\mu=0 .
$$

Now consider adding non-compact probes to this geometry frozen at positions $y=y_{i}$, as captured by the eigenvalues of a matrix $\Lambda$. As we discussed before, adding $N$ of these B-branes corresponds to turning on $N$ independent times in the space of couplings $\widetilde{t}_{n}$. To write the action on these branes we start by considering $N$ B-branes at $x=x_{i}$. In that coordinate patch this $N$-point function is simply given by the Vandermonde determinant plus the classical contribution. The classical contribution is given by

$$
\int^{x_{i}} y(x) d x=\int^{x_{i}} \mu \frac{d x}{x}=\mu \log x_{i} .
$$

We thus end up with

$$
\left\langle N\left|\psi\left(x_{1}\right) \ldots \psi\left(x_{N}\right)\right| V\right\rangle=\prod_{i<j}\left(x_{i}-x_{j}\right) e^{\sum_{i=1}^{N}\left\{-\left(i \frac{\mu}{g_{s}}\right) \log \left(x_{i}\right)+\frac{i}{g_{s}} \sum_{n>0} t_{n} x_{i}^{n}\right\}}
$$

Now we can "move" the branes to the $y$-patch. This means that the $c=1$ amplitude with both sets of times turned on equals

$$
Z\left(t_{n}, \widetilde{t}_{n}\right)=\int \prod_{i=1}^{N} d x_{i} e^{i \sum_{i} \frac{1}{g_{s}} x_{i} y_{i}}\left\langle N\left|\psi\left(x_{1}\right) \ldots \psi\left(x_{N}\right)\right| V\right\rangle
$$

We thus get a Kontsevich-like matrix model:

$$
Z\left(t_{n}, \widetilde{t}_{n}\right)=\int D X(\operatorname{det} X)^{-i\left(\mu / g_{s}\right)} e^{\left\{\frac{1}{g_{s}} \operatorname{Tr}\left(i \Lambda X+i \sum_{n} t_{n} X^{n}\right)\right\}}
$$

where $\widetilde{t}_{n}=\frac{g_{s}}{n} \operatorname{Tr} \Lambda^{-n}$. This is exactly the Imbimbo-Mukhi matrix model for $c=1$ string [41], up to identification of parameters. Namely, recall that the $c=1$ string amplitudes depend only on the ratio $\hat{\mu}$ of $\mu$ and $g_{s}, \hat{\mu}=\frac{\mu}{g_{s}}$ - this can be seen above, by rescaling appropriately the times $t_{n}, \widetilde{t}_{n}$ and $\Lambda$ by factors of $\mu$. After doing so and a suitable shift $\hat{\mu}=\frac{\mu}{g_{s}}-i N$ the agreement with [41] is manifest. The fact that these agree up to a shift in $\mu$ by $g_{N}$ is quite natural. This is because fermions shift the flux of the three-form according to (4.1), and correspondingly the value of $\mu$ depends on where one measures it. 


\subsection{General genus zero surfaces}

We can easily generalize the above formalism to more general Riemann surfaces, with many punctures. Consider for example the genus zero Riemann surface

$$
y=\frac{\prod_{i=1}^{N_{+}}\left(t_{i}-x\right)}{\prod_{j=1}^{N_{-}}\left(s_{i}-x\right)}
$$

We can assume, without loss of generality, that $N_{+}>N_{-}$. There are $N_{-}+1$ punctures corresponding to

$$
\begin{aligned}
& P: \quad x \rightarrow \infty, \quad y \rightarrow(-1)^{N_{+}+N_{-}} \infty \\
& S_{j}: \quad x \rightarrow s_{j}, \quad y \rightarrow \infty .
\end{aligned}
$$

At the $S_{j}$ punctures we use $y$ as coordinates, and at $P$, we can use $x$, for example.

The Ward identities, corresponding to putting branes at fixed $x$, read

$$
\int_{P} \psi^{*}(x) x^{n} \psi(x)+\sum_{j=1}^{N_{-}} \int_{S_{j}} \psi^{*}(y)\left(g_{s} \partial_{y}\right)^{n} \psi(y)=0 .
$$

One has, in more detail, around the $S_{j}$ puncture

$$
\int_{S_{j}} \psi_{q u}^{*}(y) e^{-\phi_{c l, j}(y) / g_{s}}\left(g_{s} \partial_{y}\right)^{n} e^{\phi_{c l, j}(y) / g_{s}} \psi_{q u}(y)
$$

where

$$
x=x_{j}(y)=\partial_{y} \psi_{c l, j}(y)=s_{j}+O\left(y^{-1}\right)
$$

is the corresponding classical solution near the $j$-th puncture. Correspondingly, it is clear that, when no B-branes are placed at $S_{j}$ punctures, the classical solution for the B-brane at the $P$-puncture is exact. Namely, the $S_{j}$ punctures contribute identically zero to the Ward identity, whereas the contribution of the $P$-puncture reads

$$
\partial_{n}^{P} \mathcal{F}=0
$$

Correspondingly, the free energy is completely trivial in this case, and the B-brane action is given by its classical value

$$
\Psi(x)=\langle 1|\psi(x)| V\rangle=e^{S(x) / g_{s}}
$$

where

$$
S(x)=\int^{x} y(x) d x .
$$


In the same case, note that the B-brane is trivially annihilated by the Riemann surface Hamiltonian:

$$
H\left(x, y=g_{s} \partial_{x}\right) \Psi(x)=0 .
$$

From this we get a Kontsevich-like matrix model describing general non-normalizable deformations of the Riemann surface corresponding to placing B-branes at various punctures. This is given by

$$
\int d X_{1} \ldots d X_{N_{-}} \prod_{i<j} \operatorname{det}\left(X_{i} \otimes 1_{j}-1_{i} \otimes X_{j}\right) e^{\sum_{1=1}^{N_{-}}\left[-\operatorname{Tr} \Lambda_{i} X_{i}+\operatorname{Tr} W\left(X_{i}\right)\right] / g_{s}}
$$

Where

$$
W(x)=S(x)+\sum_{n} t_{n} x^{n}
$$

and $S(x)$ is given by $(5.12)$.

\subsection{Example 3: Topological string on $\mathbf{P}^{1}$}

As we discussed before, if we consider the topological B-model coupled to a chiral field with superpotential $W(p)$ then this is equivalent to considering topological strings on the Calabi-Yau threefold

$$
z w-x-W(p)=0
$$

We can use this idea to solve the topological A-model string on $\mathbf{P}^{1}$ : It is known [10] that mirror symmetry relates the topological A-model on $\mathbf{P}^{1}$ to a LG theory with superpotential

$$
W(u)=e^{u}+q e^{-u}
$$

where $q=e^{-t}$ where $t$ is the Kähler parameter of $\mathbf{P}^{1}$ and $p=e^{u} \in \mathbf{C}^{*}$. We are thus led to consider topological strings on a Calabi-Yau threefold

$$
z w-x-e^{u}-q e^{-u}=0
$$

and corresponding to this a chiral boson on the Riemann surface $H(x, u)=0$ with

$$
H(x, u)=x+e^{u}+q e^{-u}
$$

Deforming the A-model theory by gravitational descendants of the Kähler class and the identity operator should correspond to inserting appropriate D-branes in this geometry. 
To start with, consider insertion of a D-brane at fixed values of $x$ and the associated one-point function $\Psi(x)=\langle\psi(x)\rangle$. As we discussed above, as operators acting on the D-brane, $x$ and $u$ are canonically conjugate $[x, u]=g_{s}$, where we can take $u=-g_{s} \partial / \partial x$. Moreover the wave function $\Psi(x)$ is annihilated by $H=H(x, u)$ :

$$
H \Psi(x)=0,
$$

with generalized Schrödinger operator

$$
H=e^{g_{s} \partial_{x}}+x+q e^{-g_{s} \partial_{x}}
$$

(equivalently, by shifting $x$ by a constant $z, \Psi(x)$ is an eigenfunction of the Lax operator $H$ with eigenvalue $z$ ). According to our philosophy the Hamiltonian $H$ is the Lax operator of an integrable hierarchy, and the corresponding flows are related to inserting many D-branes at fixed values of $x$. As it turns out, this can be identified with turning on gravitational descendants $\sigma_{n}(\omega)$ of the Kähler class $\omega$ of $\mathbf{P}^{1}$.

It has been known for a while that the $\mathbf{P}^{1}$ theory is described by a Toda lattice hierarchy [42]. Moreover, the Lax operator $L$ of the hierarchy [42], in the small phase space, coincides with $H$ in (5.14). The flows of this hierarchy generated by $\left(L^{n}\right)_{+}, n>0$ correspond to deforming by the descendants of Kähler class: the coupling constants $t_{n, \omega}$ of $\sigma_{n}(\omega)$ are related to Toda times $t_{n}$ by $t_{n}=\frac{1}{n} t_{n-1, \omega}$ [42]. (The flows corresponding to turning on descendants of the identity operator are not the usual Toda flows, but a generalization thereof [42].) We thus have shown that inserting D-branes at $x=x_{i}$, $i=1, \ldots, N$ corresponds to turning on

$$
t_{n-1, \omega}=n \sum_{i} x_{i}^{-n}
$$

Alternatively, by thinking about the $\mathbf{P}^{1}$ in bosonic terms, we can make contact with another description of the $\mathbf{P}^{1}$ theory presented in [43].

Note that the Riemann surface (5.13) has two asymptotic infinities corresponding to $x \rightarrow \infty$ and either $u \rightarrow \infty$ or $\widetilde{u}=-t-u \rightarrow \infty$. Correspondingly we have, in principle, two chiral bosons $\phi(u)$ and $\widetilde{\phi}(\widetilde{u})$ which correspond to deforming $x$ from the $u$ patch and the $\widetilde{u}$ patch as $x=\partial \phi(u)=\partial \phi_{c l}(u)+\partial \phi_{q u}(u)$ and $x=\partial \widetilde{\phi}(\widetilde{u})=\partial \widetilde{\phi}_{c l}(\widetilde{u})+\partial \widetilde{\phi}_{q u}(\widetilde{u})$, respectively where

$$
\partial \phi_{c l}(u)=e^{u}+q e^{-u}
$$


and

$$
\partial \widetilde{\phi}_{c l}(\widetilde{u})=q e^{-\widetilde{u}}+e^{\widetilde{u}}
$$

The bosons have mode expansions

$$
\partial \phi_{q u}(u)=\sum_{n>0} s_{n} e^{n u}+n \frac{\partial}{\partial s_{n}} e^{-n u}
$$

and

$$
\partial \widetilde{\phi}_{q u}(\widetilde{u})=\sum_{n>0} \widetilde{s}_{n} e^{n \widetilde{u}}+n \frac{\partial}{\partial s_{n}} e^{-n \widetilde{u}} .
$$

The action of the $\mathcal{W}_{1+\infty}$ algebra generators fixes the partition function $Z$, since it relates the modes on the two asymptotic ends. Consider for example the generator corresponding to $e^{n u} x^{m}$ for $m, n>0$. That this generates a symmetry implies that, acting on $Z=e^{\mathcal{F}}$

$$
\int_{u \rightarrow \infty} e^{n u} e^{\phi(u)} \partial^{m} e^{-\phi(u)}=\int_{\widetilde{u} \rightarrow \infty} q^{n} e^{-n \widetilde{u}} e^{\widetilde{\phi}(\tilde{u})} \partial^{m} e^{-\widetilde{\phi}(\widetilde{u})}
$$

so that one finds

$$
n \partial_{n} \mathcal{F}=q^{n} \widetilde{s}_{n}
$$

More generally, the $\mathcal{W}_{1+\infty}$ symmetry and the above Ward identities imply that

$$
\partial \phi(-t-\widetilde{u})=\partial \widetilde{\phi}(\widetilde{u})
$$

holds as an operator equation, i.e. that the theory is that of a globally defined free chiral scalar on a cylinder of length $t$. The free energy is therefore quadratic

$$
\mathcal{F}\left(s, \widetilde{s}, q, g_{s}\right)=\sum_{n>0} \frac{q^{n}}{n} s_{n} \widetilde{s}_{n}
$$

and the corresponding partition function is given by

$$
Z=\left\langle s\left|q^{L_{0}}\right| \widetilde{s}\right\rangle
$$

However, as we discussed above, the times $s_{n}, \widetilde{s}_{n}$ bear no direct relation to turning on the descendants of the Kähler class, since the modes of $\phi(u)$ correspond to insertions of B-branes at fixed $u$, whereas the descendants correspond to inserting B-branes at fixed $x$.

We can remedy this easily in the following way. It suffices to consider placing Dbranes near only one of the two punctures. Let $W$ be the operator that corresponds to 
the transformation $(x, u) \rightarrow(-u, x)$. The partition function corresponding to $\mathbf{P}^{1}$ with arbitrary descendants is now given by

$$
Z=\left\langle s\left|W^{-1} q^{L_{0}} W^{*}\right| \widetilde{s}\right\rangle
$$

By the definition of $W$ we have

$$
W \cdot \exp \left[\sum_{k>0} s_{k} \alpha_{k}\right] \cdot W^{-1}=\exp \left[\sum_{k>0} \frac{t_{k}}{(k+1) !} \hat{W}_{0}^{k+2}\right]
$$

since $\alpha_{k}$ generates the transformation $(x, u) \rightarrow\left(x+e^{k u}, u\right)$, and $\hat{W}_{0}^{k+2}$ generates $(x, u) \rightarrow$ $\left(x, u+x^{k}\right)$.

More precisely, $\hat{W}$ generates the corresponding transformation, in the background corresponding to the Riemann surface, so that it is the dressed operator,

$$
\hat{W}_{0}^{k+1}=\int d u \psi_{q u}^{*}(u) e^{-\phi_{c l}(u)} \partial^{k} e^{\phi_{c l}(u)} \psi_{q u}(u)
$$

or equivalently,

$$
\hat{W}_{0}^{k+1}=e^{-q \alpha_{-1}} e^{\alpha_{1}} W_{0}^{k+1} e^{-\alpha_{1}} e^{q \alpha_{-1}}
$$

where $W_{0}^{k+1}=\int d u \psi_{q u}^{*}(u) \partial^{k} \psi_{q u}(u)$ is the standard generator.

The operator $\hat{W}^{*}$ is the corresponding operator coming from the $\tilde{u}$ puncture which gives rise to

$$
\hat{W}_{0}^{*, k+1}=e^{q \alpha_{1}} e^{-\alpha_{-1}} W_{0}^{*, k+1} e^{\alpha_{-1}} e^{-q \alpha_{1}}
$$

where

$$
W^{*} \cdot \exp \left[\sum_{k>0} \tilde{s}_{k} \alpha_{-k}\right] \cdot W^{*-1}=\exp \left[\sum_{k>0} \frac{\tilde{t}_{k}}{(k+1) !} \hat{W}_{0}^{*, k+1}\right] .
$$

Correspondingly, the partition function of $\mathbf{P}^{1}$, deformed by the descendants of the Kähler class is, up to a constant factor given by

$$
Z=\left\langle 0\left|e^{\alpha_{1}} e^{\sum_{k>0} \frac{t_{k}}{(k+1) !} W_{0}^{k+1}} q^{L_{0}} e^{\sum_{k>0} \frac{\widetilde{t}_{k}}{(k+1) !} W_{0}^{k+1}} e^{\alpha_{-1}}\right| 0\right\rangle
$$

where we have used that

$$
e^{-\alpha_{1}} e^{q \alpha_{-1}} q^{L_{0}} e^{q \alpha_{1}} e^{-\alpha_{-1}}=q^{L_{0}} e^{q} .
$$


Since $L_{0}$ and $W_{0}^{k+1}$ commute, there is effectively only one set of times, i.e. without loss of generality, we can set $\widetilde{t}_{k}=0$, so we can write

$$
Z_{\mathbf{P}}^{1}=\left\langle 0\left|e^{\alpha_{1}} e^{\sum_{k>0} \frac{t_{k}}{(k+1) !} W_{0}^{k+1}} q^{L_{0}} e^{\alpha_{-1}}\right| 0\right\rangle,
$$

Note that the partition function is identical to the partition function for the $\mathbf{P}^{1}$ obtained in [43], eqn. 3.13, which is a nice verification of our formalism.

We can also conjecture a matrix model that describes this. Very analogous to the Kontsevich matrix model of the $(p, 1)$ bosonic minimal models, we can give a simple matrix model description for the $\mathbf{P}^{1}$ A-model topological string by making use of the simplicity of the D-brane amplitudes in the $u$-patch. There, $x=g_{s} \partial / \partial u$ and the corresponding one-point function $\Psi(u)$ satisfies $H \Psi(u)=0$ with

$$
H=g_{s} \frac{\partial}{\partial u}-e^{u}-q e^{-u} .
$$

Since the Hamiltonian is linear in the momentum, the semi-classical approximation is exact, and

$$
\Psi(u)=e^{\left(e^{u}-q e^{-u}\right) / g_{s}} .
$$

The matrix model action corresponding to inserting $N$ D-branes at fixed positions $x_{i}$ (now considered as the eigenvalues of a matrix $X$ ) is

$$
\left.Z_{\mathbf{P}^{1}}(X)=\int d_{H} U \exp \operatorname{Tr}\left(X U-e^{U}-q e^{-U}\right) / g_{s}\right]
$$

where $U$ is a Hermitian matrix and $d_{H} U$ is the measure induced from the Haar measure on the $U(N)$ group manifold on its tangent space (respecting the periodicity $U \rightarrow U+$ $2 \pi i)$. Here the traces $\frac{1}{n} \operatorname{Tr} X^{-n}=t_{n}$ correspond to the Toda times. We conjecture that this matrix model is describing topological A-model of the $\mathbf{P}^{1}$ when the couplings to the descendants of the identity operator are turned off. This should also be equivalent to the matrix model of [42]. It is an interesting question to understand what configuration of B-branes corresponds to turning on these more general couplings as well.

One can also deform the theory by turning on a twisted mass $\tau$ on $\mathbf{P}^{1}$ which gives an equivariant version of the topological string on $\mathbf{P}^{1}$. In this case the superpotential gets deformed to 10

$$
W(u)=e^{u}+q e^{-u}+\tau u,
$$

and the corresponding Calabi-Yau becomes

$$
z w-x-e^{u}-q e^{-u}+\tau u=0 .
$$

The considerations here should proceed much in the same way as in the non-equivariant case, and it would be nice to work out the details. 


\subsection{Example 4: Integrable structure of the topological vertex}

The topological vertex was introduced in [9] as the building block for topological string amplitudes on local, toric Calabi-Yau threefolds (for further properties and applications of the topological vertex, see for example [44,45,46,47,48,49]). In terms of the topological A-model, the vertex can be regarded as an open string amplitude in the $\mathbf{C}^{3}$ geometry with boundaries on certain Lagrangian A-branes. In [9] the vertex amplitude is described by the partition function

$$
Z\left(\Lambda_{u}, \Lambda_{v}, \Lambda_{w}\right)=\sum_{R_{i}} C_{R_{1} R_{2} R_{3}} \operatorname{Tr}_{R_{1}} \Lambda_{u} \operatorname{Tr}_{R_{2}} \Lambda_{v} \operatorname{Tr}_{R_{3}} \Lambda_{w}
$$

where we are choosing the canonical framing. The entries $C_{R_{1} R_{2} R_{3}}$ were obtained in [9] from large $N$ duality with Chern-Simons theory, and the result is

$$
C_{R_{1} R_{2} R_{3}}=\sum_{R, Q_{1}, Q_{3}} N_{Q_{1} R}^{R_{1}} N_{Q_{3}^{t} R}^{R_{3}^{t}} q^{\kappa_{R_{2}} / 2+\kappa_{R_{3}} / 2} \frac{W_{R_{2}^{t} Q_{1}} W_{R_{2} Q_{3}^{t}}}{W_{R_{2}}}
$$

where $W_{R_{1} R_{2}}$ is the large $N$ limit of the CS invariant of the Hopf link, as defined in [50,9], and $W_{R}=W_{R}$, where - denotes the trivial representation, is the large $N$ limit of the

quantum dimension of $R$. In the above equation, $N_{R_{1} R_{2}}^{R_{3}}$ are tensor product coefficients, and $\kappa_{R}$ is given by

$$
\kappa_{R}=\sum_{i} \ell_{i}\left(\ell_{i}-2 i+1\right)
$$

where $\ell_{i}$ are the lengths of rows in the Young tableau associated to $R$. We can consider the topological vertex in an arbitrary framing (we will discuss framing from the B-model perspective in the next subsection),

$$
C_{R_{1} R_{2} R_{3}}^{\left(n_{1}, n_{2}, n_{3}\right)}=(-1)^{\sum_{i} n_{i} \ell\left(R_{i}\right)} q^{\sum_{i} n_{i} \kappa_{R_{i}} / 2} C_{R_{1} R_{2} R_{3}}
$$

where $\ell(R)$ is the total number of boxes in the representation $R$. We note that $\kappa_{R}$, that implements the change of framing, is the eigenvalue of the operator $\oint(\partial \phi)^{3} / 3$ acting on the state $|R\rangle$, as it follows from our discussion of framing in section 3.1. The free energy associated to the vertex is given by $\mathcal{F}\left(g_{s}, t_{n}^{i}\right)=\log Z\left(g_{s}, t_{n}^{i}\right)$, where the parameters $t_{n}^{i}$ are related to the three matrices $\Lambda_{i}$ appearing in (5.16) by $t_{n}^{i}=\frac{g_{s}}{n} \operatorname{Tr} \Lambda_{i}^{n}, i=u, v, w$. By using the representation (3.4), it is easy to see that $\oint(\partial \phi)^{3} / 3$ is the "cut and join" operator considered for example in [19], and the free energy associated to the framed vertex satisfies then three "cut and join" equations (one for each framing $n_{i}$ ). 
We want to describe now the topological vertex in the topological B-model. We first consider the canonical framing $n_{i}=0$. Mirror symmetry relates the A-model $\mathbf{C}^{3}$ geometry to Kodaira-Spencer theory on the Calabi-Yau manifold

$$
z w-e^{-u}-e^{v}+1=0 .
$$

As explained in [9], this is described by a chiral boson on the Riemann surface

$$
e^{-u}+e^{v}-1=0 .
$$

In [9] some evidence for this was presented. Here we will show how to recover in the B-model the full free energy of the topological vertex, following the general philosophy developed in the previous sections. Following the discussion of sections 3 and 4 we will show that we can completely recover the topological A-model amplitudes by considering the chiral boson on the above Riemann surface. Moreover, the fermionic perspective provides a remarkably simple description of the vertex in terms of a wave function quadratic in the fermions. We will first develop both the bosonic and the fermionic viewpoint and then we will demonstrate that this indeed agrees with the A-model amplitudes derived in [9].

The mirror Riemann surface (5.20) is a sphere with three punctures, i.e. three asymptotic regions. We will choose the three punctures have coordinates $u, v$ and $w$ on them, where

$$
u+v+w=i \pi
$$

The $u$ coordinate parameterizes the asymptote of the Riemann surface where $u \rightarrow \infty$, and likewise for the $v$ - and $w$-punctures. These coordinates are defined on a cylinder and have periodicity $2 \pi i$. With this choice of variables, the Riemann surface has a $\mathbf{Z}_{3}$ symmetry corresponding to cyclic permutations

$$
u \rightarrow v \rightarrow w \rightarrow u .
$$

There are three corresponding chiral scalars deforming the complex structure. In the $u$-patch, the meromorphic one form is $\lambda=v d u$, and the chiral boson representing the variations of this can be identified as $v=\partial_{u} \phi^{u}$. Correspondingly, in terms of the fermion quantum mechanics,

$$
[v, u]=g_{s}
$$


with

$$
v=g_{s} \partial_{u} .
$$

We should emphasize here that in the case of Riemann surfaces which correspond to mirrors of topological A-model there is always a preferred choice of which variable corresponds to momentum $p$ at each puncture [14]. This is because the classical value of $\phi(u)$ corresponds to the disk amplitude in the topological A-model. The physical part of this amplitude is generated by worldsheet instantons of complexified area $u$ whose contribution vanishes in the $u \rightarrow \infty$ limit as $e^{-u}$. This means that near the $u$-puncture, the classical B-brane action is

$$
S(u)=\phi(u) / g_{s}=\frac{1}{g_{s}} \int^{u} p(u) d u .
$$

where $p(u)$ is a variable defined by asking that on the Riemann surface $p$ vanishes as $u \rightarrow \infty$. In the present case, we have that in the $u$-patch, the corresponding momentum is $p_{u}=\partial_{u} \phi^{u}=v$. Similarly in the $v$ and $w$ patches, we have

$$
w=p_{v}=\partial_{v} \phi^{v}, \quad u=p_{w}=\partial_{w} \phi^{w}
$$

so that, for example

$$
u=i \pi-v-g_{s} \partial_{v}=g_{s} \partial_{w},
$$

Let us now discuss the $\mathcal{W}_{1+\infty}$ relations. The chiral boson defined in the $u$ patch has an expansion

$$
\phi(u)=\sum_{n>0} t_{n}^{u} e^{n u}-g_{s}^{2} \frac{1}{n} \frac{\partial}{\partial t_{n}^{u}} e^{-n u},
$$

corresponding to turning on a coherent state, and we have a corresponding fermion

$$
\psi(u)=e^{\phi(u) / g_{s}}
$$

Consider now the fermion bilinear operator

$$
\oint_{u} \psi^{*}(u) e^{n u} \psi(u)
$$

Moving the contour to the other two patches, and using that the operator $u$ is represented in the $v$ - and $w$-patches by (5.21) we find the Ward identity

$$
\oint_{u} \psi^{*}(u) e^{n u} \psi(u)+(-1)^{n} \oint_{v} \psi^{*}(v) e^{-n v-n g_{s} \partial_{v}} \psi(v)+\oint_{w} \psi^{*}(w) e^{n g_{s} \partial_{w}} \psi(w)=0
$$


There is in fact a triple infinity of conserved charges obtained from the above by the cyclic $\mathbf{Z}_{3}$ symmetry of the problem. Bosonization of (5.24) gives

$$
\oint_{u} e^{n u} \partial_{u} \phi(u) / g_{s} d u=\oint_{v} \frac{(-1)^{n}}{[n]} e^{-n v-n \Delta_{v} \phi(v)} d v-\oint_{w} \frac{1}{[n]} e^{n \Delta_{w} \phi(w)} d w .
$$

where we have defined, e.g.:

$$
n \Delta_{v} \phi(v):=\frac{1}{g_{s}}\left(\phi\left(v+n g_{s} / 2\right)-\phi\left(v-n g_{s} / 2\right)\right)
$$

and introduced the $q$-number 6

$$
[n]=q^{\frac{n}{2}}-q^{-\frac{n}{2}}
$$

with $q=e^{g_{s}}$. These Ward identities, viewed as differential equations for the free energy $\mathcal{F}\left(t^{u}, t^{v}, t^{w}\right)$, are strong enough to determine it uniquely.

Let us first discuss the classical limit, $g_{s} \rightarrow 0$. In this limit we have that

$$
e^{\Delta_{u} \phi(u) / g_{s}} \rightarrow e^{\partial_{u} \phi(u)}
$$

and only the genus zero piece $F_{0}$ of the free energy $\mathcal{F}(t)=\sum_{g} F_{g}(t) g_{s}^{2 g-2}$ survives, e.g.

$$
\left\langle t\left|e^{\Delta^{u} \phi^{u}}\right| V\right\rangle_{g_{s} \rightarrow 0}=\exp \left\{\sum_{n>0} n t_{n}^{u} e^{n u}+\partial_{n}^{u} F_{0} e^{-n u}\right\} .
$$

so that the Ward identity becomes

$$
\oint_{u} e^{n u} \partial_{u} \phi^{u} d u=\oint_{v} \frac{(-1)^{n}}{n} e^{-n v-n \partial_{v} \phi^{v}} d v-\oint_{w} \frac{1}{n} e^{n \partial \phi^{w}(w)} d w .
$$

The semiclassical limit could have equivalently been obtained by recalling that, e.g. $\partial^{u} \phi(u)=v$ and then considering

$$
\oint_{u \rightarrow \infty} e^{n u} v d u
$$

6 The appearance of $1 /[n]$ in the r.h.s. of (5.25) comes from the normal-ordering factor which is given by $\exp \left(\sum_{k>0}[n k]^{2} /(2 k)\right)$. This sum contains a finite piece

$$
\exp \left(\frac{1}{2} \sum_{k>0} \frac{q^{n k}+q^{-n k}}{k}\right)=-\frac{1}{[n]}
$$

which should be included after normal ordering. 
moving the contour to the other patches and integrating by parts. This classical Ward identity determines the genus zero free energy. For example, the leading order solution for $F_{0}$, corresponding to genus zero with one hole is obtained by setting all the times to zero to get

$$
\partial_{n}^{u} F_{0}=-\frac{1}{n}
$$

Note that this is computing

$$
v=\partial_{u} \phi_{c l}^{u}=-\sum_{n} \frac{1}{n} e^{-n u}=\log \left(1-e^{-u}\right),
$$

which corresponds to the classical equation of the Riemann surface. The case of Riemann surfaces which correspond to mirrors of the toric topological A-model geometries is special, namely the knowledge of the location of the punctures in terms of giving the momenta and the coordinates, suffices to fix the Riemann surface itself. It is thus not surprising that the same data fixes the quantum one-point amplitudes as well, as we will soon see. Expanding about the one-point function, $\phi_{c l}$ it is easy to see that the two point functions precisely correspond to a global chiral scalar on the Riemann surface (5.20) in accordance with [9]. The full theory is much more complicated than that, even in the classical limit, and $F_{0}$ in general has non-zero coefficients for all powers of the $t$ 's.

We can now use the quantum Ward identity (5.25) to solve for the full free energy. A priori, we should solve the identity iteratively order by order in the genus expansion. One can however do better than this and obtain exact expressions to all genera, proceeding iteratively in the number of times turned on. Let us now explain this.

The equation (5.25), after acting on $Z$, leads to the following identity:

$$
\begin{aligned}
g_{s} \partial_{n}^{u} \mathcal{F}= & \frac{(-1)^{n}}{[n]} \oint_{v} d v e^{-n v} e^{\left\{\sum_{m>0}-g_{s}^{-1}[m n] t_{m}^{v} e^{m v}-\Delta_{v, n} \mathcal{F}(t)\right\}} \\
& -\frac{1}{[n]} \oint_{w} d w e^{\left\{\sum_{m>0} g_{s}^{-1}[m n] t_{m}^{w} e^{m w}+\Delta_{w, n} \mathcal{F}(t)\right\}},
\end{aligned}
$$

where we defined

$$
\begin{aligned}
\Delta_{v, n} \mathcal{F}(t) & :=\mathcal{F}\left(t^{u}, t_{m}^{v}-g_{s} \frac{[m n]}{m} e^{-m v}, t^{w}\right)-\mathcal{F}\left(t^{u}, t^{v}, t^{w}\right) \\
& =\sum_{\ell=1}^{\infty} \sum_{q_{1}, \cdots, q_{\ell}} \frac{\left(-g_{s}\right)^{\ell}}{\ell !} \prod_{i=1}^{\ell} \frac{\left[n q_{i}\right]}{q_{i}} \partial_{q_{1}}^{v} \cdots \partial_{q_{\ell}}^{v} \mathcal{F}\left(t^{u}, t^{v}, t^{w}\right) e^{-\sum_{i=1}^{\ell} q_{i} v}
\end{aligned}
$$


i.e. we expand the free energy $\mathcal{F}\left(t^{u}, t_{m}^{v}-g_{s}[m n] e^{-m v} / m, t^{w}\right)$ on the r.h.s. in the times around $\mathcal{F}\left(t^{u}, t^{v}, t^{w}\right)$, and similarly for the term in the $w$ integral. Using (5.28) and the cyclic property of the vertex it is straightforward to provide an algorithm to calculate the exact coefficients in $q$ of the monomial $t_{\vec{k}}^{u} t_{\vec{m}}^{v} t_{\vec{n}}^{w}$ in the free energy $\mathcal{F}$ to any given order in the times and total "winding".

For example, it is easy to see that the exact amplitude corresponding to times on the $u$-puncture is given by

$$
\mathcal{F}\left(t^{u} ; t^{v}=0=t^{w}\right)=-\sum_{n=1}^{\infty} \frac{t_{n}^{u}}{g_{s}[n]}
$$

as follows by integrating (5.28) with respect to $t_{n}^{u}$. For $t^{v}=t^{w}=0$ the only term that contributes from the righthand side is the constant $-\frac{1}{[n]}$ coming from the second term.

Similarly the coefficients of $t_{k_{1}}^{u} \ldots t_{k_{s}}^{u} t_{m_{1}}^{v} \ldots t_{m_{n}}^{v}$ and $t_{k_{1}}^{u} \ldots t_{k_{s}}^{u} t_{n_{1}}^{w} \ldots t_{n_{r}}^{w}$ are obtained by integrating (5.28) with respect to $t_{k_{i}}^{u}$ and iteration over $s$. Each step can be performed to arbitrary order in the total number of holes $N_{v, w}=\sum_{i=1}^{r} k_{i}^{v, w}$. Explicit formulas for certain amplitudes to arbitrary winding can be obtained, see appendix A. Checks on the cyclic property are provided by comparison of the coefficients of $t_{\vec{k}}^{u} t_{\vec{n}}^{w} \underset{\text { cycl. }}{\longrightarrow} t_{\vec{k}}^{w} t_{\vec{n}}^{v}$ with the one of $t_{\vec{k}}^{u} t_{\vec{n} \text { cycl. }}^{v} t_{\vec{k}}^{v} t_{\vec{n}}^{w}$. These coefficients can be used to obtain $t_{k_{1}}^{u} \ldots t_{k_{s}}^{u} t_{m_{1}}^{v} \ldots t_{m_{n}}^{v} t_{n_{1}}^{w} \ldots t_{n_{r}}^{w}$ again by integration (5.23) with respect to $t_{k_{i}}^{u}$ and iteration in $s$. This has been done to order $\left(N_{u}, N_{v}, N_{w}\right)=(4,4,4)$ in complete agreement with the free energy from the Chern-Simons formula for the vertex (5.17).

The underlying integrable structure of the topological vertex is better formulated in the fermionic language, and it implies that albeit the vertex has a rather complicated structure when written in terms of modes of a chiral boson, after fermionization it is simply given by the exponential of a bilinear of fermions acting on the vacuum. We turn to this next.

\subsection{Fermion perspective for the topological vertex}

Let us now consider the fermionic description. From the above, it follows that the fermion one point function

$$
\Psi(u)=\left\langle 0\left|e^{\phi(u) / g_{s}}\right| V\right\rangle
$$

is given by the so-called quantum dilogarithm [51]

$$
\Psi(u)=\exp \sum_{n>0}-\frac{e^{-n u}}{n[n]} .
$$


Other useful ways of writing this are

$$
\exp \sum_{n>0}-\frac{e^{-n u}}{n[n]}=\prod_{n=0}^{\infty}\left(1-e^{-u} q^{n+\frac{1}{2}}\right)^{-1}=\sum_{n \geq 0}(-1)^{n} \frac{1}{[n] !} q^{-n(n-1) / 4} e^{-n u}
$$

The one point function is annihilated by the Hamiltonian corresponding to the Riemann surface (5.20), leading to a difference equation which uniquely fixes it

$$
H(u, v) \Psi(u)=0
$$

where

$$
H(u, v)=q^{-\frac{1}{2}} e^{-u}+e^{g_{s} \partial_{u}}-1 .
$$

This reflects, as discussed in section 3, the unbroken $\mathcal{W}$ symmetries. For the anti-brane a similar reasoning gives $\prod_{n=0}^{\infty}\left(1-e^{-u} q^{n+\frac{1}{2}}\right)$. As we discussed in section 4 , the B-branes transform as wave functions in going from patch to patch. With coordinates on the punctures chosen as in the previous subsection, going from the $u$ to the $v$ patch the coordinates transform by a symplectic transformation $U$ which, up to a shift, corresponds to the $S T$ element of the $S L(2, \mathbf{Z})$.

$$
\begin{aligned}
U\left(\begin{array}{l}
v \\
u
\end{array}\right) & =S T\left(\begin{array}{l}
v \\
u
\end{array}\right)+\left(\begin{array}{c}
0 \\
i \pi
\end{array}\right) \\
S T & =\left(\begin{array}{cc}
0 & 1 \\
-1 & -1
\end{array}\right)
\end{aligned}
$$

The element $S T$ generates a $\mathbf{Z}_{3}$ subgroup of $S L(2, \mathbf{Z}),(S T)^{3}=1$, and similarly $U^{3}=$ id.

In terms of the fermions, these are realized as

$$
\begin{aligned}
U \psi(v) & =\int d u e^{-S_{U}(v, u) / g_{s}} \psi(u) \\
U^{2} \psi(w) & =\int d u e^{-S_{U^{2}}(w, u) / g_{s}} \psi(u)
\end{aligned}
$$

where

$$
S_{U}(v, u)=-\frac{v^{2}+2 u v+2 i \pi v}{2} .
$$

Similarly from $w$ to $u$ patches there is a generating function

$$
\begin{aligned}
& S_{U}(u, w)=-\frac{u^{2}+2 w u+2 i \pi u}{2} . \\
& S_{U^{2}}(w, u)=\frac{u^{2}+2 u w+2 i \pi u}{2} .
\end{aligned}
$$


Note that $S_{U^{2}}(w, u)=-S_{U}(u, w)$, and correspondingly, it is easy to check that $U^{3}$ is realized as identity on the fermions.

In this example it is easy to check that the one-point functions $\Psi(u)=\langle\psi(u)\rangle$ satisfy

$$
\Psi(w)=\int d u e^{-S_{U^{2}}(w, u) / g_{s}} \Psi\left(u+g_{s} / 2\right),
$$

by performing a Gaussian integral over $u$, and analytically continuing in $w$, to $w \rightarrow \infty$ patch. That is, up to the shift in $u$ we have $\Psi(w)=U^{2} \Psi(w)$. The shift in $u$ on the right hand side is a quantum shift in the flat coordinate. While in the case of pure topological gravity, for example, one could derive very useful statements by considering how fermions transform from patch to patch, here the one point functions are complicated in every patch. Incidentally, the statement that the quantum dialogarithm is its own Fourier transform is well known [51].

The expression or $|V\rangle$ derived in the previous subsection in terms of the bosons is hiding the amazing simplicity of the state $|V\rangle$ - it is a Bogoliubov transform of the fermionic vacuum! Namely, we have that

$$
|V\rangle=\exp \left[\sum_{i, j, n, m} a_{m n}^{i j} \psi_{-m-1 / 2}^{i} \psi_{-n-1 / 2}^{j *}\right]|0\rangle
$$

where, due to the cyclicity of the vertex, the coefficients $a_{m n}^{i j}$ must have the cyclic symmetry

$$
a_{m n}^{i j}=a_{m n}^{i+1, j+1}
$$

so that there are only three independent matrices of coefficients, $a^{u u}, a^{u v}$ and $a^{v u}$. That this is true can be shown as follows. Note that if we consider B-branes in one patch only, than the corresponding amplitude is manifestly bilinear in the fermions. This is because the corresponding state in terms of the bosons is simply a coherent state, which is the exponential of a bilinear in fermions acting on the vacuum. Since the fermions transform linearly from patch to patch, this implies that the complete state $|V\rangle$ is bilinear in the fermions. Moreover, the coefficients $a^{i j}$ are easily found if one knows the amplitudes in one patch.

Since the exact free energy (5.29) corresponding to one puncture $\mathcal{F}\left(t^{u}, 0,0\right)$ is linear in the times $t_{n}^{u}$, we have that $G(\tilde{u}, u)=\frac{1}{e^{\tilde{u}}-e^{u}}$ is the standard fermion Green function on a plane:

$$
\left\langle 0\left|\psi(\tilde{u}) \psi^{*}(\tilde{u})\right| V\right\rangle=\frac{1}{e^{\tilde{u}}-e^{u}} \Psi(\tilde{u}) \Psi^{*}(u)=\frac{1}{e^{\tilde{u}}-e^{u}} \prod_{n=0}^{\infty}\left(\frac{1-e^{-u} q^{n+1 / 2}}{1-e^{-\tilde{u}} q^{n+1 / 2}}\right)
$$


This can be used to compute the state $|V\rangle$, as described in section 4, namely from (5.33) we can compute $a^{u u}$ as:

$$
\left\langle 0\left|\psi(\tilde{u}) \psi^{*}(u)\right| V\right\rangle=\sum_{m, n=0}^{\infty} a_{m n}^{u u} e^{-(m+1) u} e^{-(n+1) \tilde{u}}+\frac{1}{e^{\tilde{u}}-e^{u}}
$$

From this we find that:

$$
a_{m n}^{u u}=(-1)^{n} \frac{q^{\frac{m(m+1)}{4}}-\frac{n(n+1)}{4}}{[m+n+1][m] ![n] !} .
$$

We can similarly compute $a_{m n}^{v u}$ by evaluating $\left\langle 0\left|\psi(v) \psi^{*}(u)\right| V\right\rangle$ and considering how the fermions transform, namely

$$
\left\langle 0\left|\psi(v) \psi^{*}(u)\right| V\right\rangle=\int d \tilde{u} e^{-S_{U}(v, \tilde{u}) / g_{s}} \frac{\Psi\left(\tilde{u}+g_{s} / 2\right) \Psi^{*}(u)}{e^{\tilde{u}+g_{s} / 2}-e^{u}}
$$

and expanding in $e^{-u}$ and $e^{-v}$. The Gaussian integral in (5.34) gives]

$$
\left\langle 0\left|\psi(v) \psi^{*}(u)\right| V\right\rangle=e^{-u} \sum_{n \geq 0} q^{-\frac{n(n-1)}{2}}(-1)^{n+1} e^{-n v} e^{-n u} \Psi\left(v+n g_{s}\right) \Psi^{*}(u)
$$

The coefficient $a_{m n}^{v u}$ of the $e^{-m v} e^{-(n+1) u}$ term in the expression on the right hand side is

$$
a_{m n}^{v u}=(-1)^{n+1} q^{\frac{m(m-1)}{4}-\frac{n(n-1)}{4}} \sum_{p=0}^{\min (m, n)} \frac{q^{\frac{p(p-m-n+1)}{2}}}{[m-p] ![n-p] !} .
$$

Similarly, we can compute $a^{u v}$ by considering

$$
\left\langle 0\left|\psi(u) \psi^{*}(v)\right| V\right\rangle=\int d \tilde{u} e^{S_{U}(v, u) / g_{s}} \frac{\Psi(u) \Psi^{*}\left(\tilde{u}-g_{s} / 2\right)}{e^{u}-e^{\tilde{u}-g_{s} / 2}} .
$$

7 For computing this, it is useful to note that

$$
\int d u e^{-S_{U}(v, u) / g_{s}} e^{n u} \Psi\left(u+g_{s} / 2\right)=\Psi\left(v+n g_{s}\right)(-1)^{n} e^{-n v} q^{-\frac{n^{2}}{2}}
$$

To define this integral and consistently keep track of the quantum shifts, it is helpful to express it as $\int d u \int d w e^{-S_{U^{2}}(v, w) / g_{s}-S_{U^{2}}(w, u) / g_{s}} e^{n u} \Psi\left(u+g_{s} / 2\right)$ using that $U=U^{2} \cdot U^{2}$, and further making use of equation (5.31). 
Following similar steps to the ones spelled out above, (or simply taking $g_{s} \rightarrow-g_{s}$ which exchanges the branes with the anti-branes) we find

$$
a_{n m}^{u v}=(-1)^{m} q^{-\frac{m(m-1)}{4}} q^{\frac{n(n-1)}{4}} \sum_{p=0}^{\min (m, n)} \frac{q^{-\frac{p(p-m-n+1)}{2}}}{[m-p] ![n-p] !}
$$

In the next section, we will see that this agrees with the previously obtained expressions for the vertex. More precisely, this agrees up to the factor of $e^{-u}$ and a shift of $v$ by a $g_{s}$ factor. The relative factors and shifts are probably related to the fact that we were not carefuly keeping track of fermion charges.

\subsection{Fermion bilinearity and the equivalence with the vertex from the topological A-model}

In this section, we show that the vertex amplitude, as computed above from the topological B-model perspective, agrees with the expression (5.17) for the vertex from the topological A-model.

As we have seen in the previous section, the vertex amplitude $|V\rangle$ (which is defined by $\left.Z\left(t^{i}\right)=\left\langle t^{i} \mid V\right\rangle\right)$ is of the form

$$
|V\rangle=\exp \left[\sum_{i, j, n, m} a_{m n}^{i j} \psi_{-m-1 / 2}^{i} \psi_{-n-1 / 2}^{j *}\right]|0\rangle .
$$

To show that this agrees with (5.17), we compute the coefficients $a_{n m}^{i j}$ in terms of the entries of the vertex in the representation basis. As it is well-known (see for example [52]), the state corresponding to a hook representation $R_{m+1, n+1}$ with $m+1$ boxes in its row and $n+1$ boxes in its column is given by

$$
\left|R_{m+1, n+1}\right\rangle=(-1)^{n+1} \psi_{-n-1 / 2}^{*} \psi_{-m-1 / 2}|0\rangle
$$

By taking the inner product with $|V\rangle$ and using (5.32) we find that

$$
C_{R_{m+1, n+1} . .}=(-1)^{n} a_{m n}^{u u}
$$

plus permutations. Similarly, we obtain

$$
C_{R_{p+1, p^{\prime}+1} R_{q+1, q^{\prime}+1}}=(-1)^{p^{\prime}+q^{\prime}}\left(a_{p p^{\prime}}^{u u} a_{q q^{\prime}}^{v v}-a_{p q^{\prime}}^{u v} a_{q p^{\prime}}^{v u}\right)
$$


plus permutations. Finally, the trivalent vertex with the reps $R_{p+1, p^{\prime}+1}, R_{q+1, q^{\prime}+1}$, $R_{r+1, r^{\prime}+1}$ in positions $k, l, m$, respectively, is given by

$$
\begin{aligned}
(-1)^{p^{\prime}+q^{\prime}+r^{\prime}} & \left(a_{p q^{\prime}}^{k l} a_{q r^{\prime}}^{l m} a_{r p^{\prime}}^{m k}+a_{p r^{\prime}}^{k m} a_{r q^{\prime}}^{m l} a_{q p^{\prime}}^{l k}-a_{p p^{\prime}}^{k k} a_{q r^{\prime}}^{l m} a_{r q^{\prime}}^{m l}\right. \\
& \left.-a_{q q^{\prime}}^{l l} a_{p r^{\prime}}^{k m} a_{r p^{\prime}}^{m k}-a_{r r^{\prime}}^{m m} a_{q p^{\prime}}^{l k} a_{p q^{\prime}}^{k l}+a_{p p^{\prime}}^{k k} a_{q q^{\prime}}^{l l} a_{r r^{\prime}}^{m m}\right)
\end{aligned}
$$

Using these equations one can determine all the coefficients $a_{n m}^{i j}$ in terms of entries of the trivalent vertex with hook representations. One first finds $a_{00}^{u v}=q^{1 / 6}, a_{00}^{v u}=-q^{-1 / 6}$. Since

$$
C_{R_{1} R_{2}}=q^{-\kappa_{R_{2}^{t}} / 2} W_{R_{1} R_{2}^{t}}
$$

one finds from (5.37) that

$$
\begin{aligned}
& a_{m n}^{u v}=(-1)^{n} q^{\frac{1}{6}-\kappa_{S_{n+1}} / 2}\left(W_{S_{m+1} S_{n+1}}-W_{S_{m+1}} W_{S_{n+1}}\right), \\
& a_{m n}^{v u}=-(-1)^{n} q^{-\frac{1}{6}-\kappa_{A_{m+1}} / 2}\left(W_{A_{m+1} A_{n+1}}-W_{A_{m+1}} W_{A_{n+1}}\right),
\end{aligned}
$$

where $S_{n}, A_{n}$ are respectively the completely symmetric and antisymmetric representations with $n$ boxes. The following formula for $W_{R_{1} R_{2}}$ is a consequence of cyclicity of the vertex (see [53] for a detailed proof):

$$
W_{R_{1} R_{2}}=q^{\left(\kappa_{R_{1}}+\kappa_{R_{2}}\right) / 2} \sum_{R} W_{R_{1}^{t} / R} W_{R_{2}^{t} / R}
$$

where

$$
W_{R / R^{\prime}}=\sum_{Q} N_{Q R^{\prime}}^{R} W_{Q}
$$

and can be written in terms of skew Schur functions [46]. Using (5.39) one easily finds

$$
\begin{aligned}
& a_{m n}^{u u}=(-1)^{n} \frac{q^{\frac{m(m+1)}{4}-\frac{n(n+1)}{4}}}{[m+n+1] ![m] ![n] !}, \\
& a_{m n}^{u v}=(-1)^{n} q^{\frac{m(m+1)}{4}-\frac{n(n+1)}{4}+\frac{1}{6}} \sum_{l=0}^{\min (m, n)} \frac{q^{\frac{1}{2}(l+1)(m+n-l)}}{[m-l] ![n-l] !}, \\
& a_{m n}^{v u}=-(-1)^{n} q^{\frac{m(m+1)}{4}-\frac{n(n+1)}{4}-\frac{1}{6}} \sum_{l=0}^{\min (m, n)} \frac{q^{-\frac{1}{2}(l+1)(m+n-l)}}{[m-l] ![n-l] !} .
\end{aligned}
$$

Notice that the fermionic representation leads to a radical simplification of the information encoded in the vertex, and to a full series of consistency relations between the coefficients $C_{R_{1} R_{2} R_{3}}$ that are the analog of the Plücker relations in the KP hierarchy. It is worth 
mentioning that the state (5.32) is a tau function of the three-component KP hierarchy as constructed for example in [54]. It follows in particular that if we put one set of times to zero we obtain a tau function of the Toda hierarchy 8 .

It is interesting to note that there is a choice of framing for which at least some of the amplitudes simplify dramatically. Suppose we consider the vertex amplitude corresponding to only two stacks of B-branes, say in the $u$ and the $v$ patches, with framing -1 in the $u$-patch and canonical framing in $v$-patch. The correlation functions we want to compute are

$$
\left\langle 0\left|\psi^{*}(u) \psi(v)\right| V^{(-1,0,0)}\right\rangle=-\sum_{m, n=0}^{\infty}(-1)^{m+1} q^{-\kappa_{S_{m+1}} / 2} a_{m n}^{u v} e^{-(m+1) u} e^{-(n+1) v},
$$

and

$$
\left\langle 0\left|\psi(u) \psi^{*}(v)\right| V^{(-1,0,0)}\right\rangle=\sum_{m, n=0}^{\infty}(-1)^{n+1} q^{-\kappa_{A_{n+1}} / 2} a_{m n}^{v u} e^{-(n+1) u} e^{-(m+1) v} .
$$

Using (5.38), we find that (5.41) can be written as

$$
\begin{aligned}
& \left\langle 0\left|\psi(u) \psi^{*}(v)\right| V^{(-1,0,0)}\right\rangle= \\
& q^{1 / 6} \sum_{m, n=0}^{\infty}\left(-e^{-u}\right)^{m+1}\left(-e^{-v}\right)^{n+1} q^{-\kappa_{S_{m+1}} / 2-\kappa_{S_{n+1}} / 2}\left(W_{S_{m+1} S_{n+1}}-W_{S_{m+1}} W_{S_{n+1}}\right) .
\end{aligned}
$$

Therefore, we just have to compute the generating function of the $W_{R_{1} R_{2}}$ for symmetric representations, in the framing $(-1,-1)$. It can be shown by using $(5.39)$ that

$$
(-1)^{\ell\left(R_{1}\right)+\ell\left(R_{2}\right)} q^{-\kappa_{R_{1}} / 2-\kappa_{R_{2}} / 2} W_{R_{1} R_{2}}=\left\langle R_{1}\right| \otimes\left\langle R_{2} \mid W\right\rangle
$$

where the state $|W\rangle$ in $\mathcal{H}^{(1)} \otimes \mathcal{H}^{(2)}$ is given by

$$
|W\rangle=\exp \left\{-\sum_{n=1}^{\infty} \frac{\beta_{n}}{n}\left(\alpha_{-n}^{(1)}+\alpha_{-n}^{(2)}\right)+\sum_{n=1}^{\infty} \frac{1}{n} \alpha_{-n}^{(1)} \alpha_{-n}^{(2)}\right\}|0\rangle,
$$

where $\beta_{n}=\frac{1}{[n]}$. Using (5.42) one finds

$$
\left\langle 0\left|\psi^{*}(u) \psi(v)\right| V^{(-1,0,0)}\right\rangle=-\frac{q^{1 / 6}}{1-e^{u+v}} \prod_{n \geq 0}\left(1-e^{-u} q^{n+\frac{1}{2}}\right)^{-1}\left(1-e^{-v} q^{n+\frac{1}{2}}\right)^{-1}
$$

8 This fact has also been pointed out in [55]. 
Similarly,

$$
\left\langle 0\left|\psi(u) \psi^{*}(v)\right| V^{(-1,0,0)}\right\rangle=-\frac{q^{-1 / 6}}{1-e^{u+v}} \prod_{n \geq 0}\left(1-e^{-u} q^{n+\frac{1}{2}}\right)\left(1-e^{-v} q^{n+\frac{1}{2}}\right) .
$$

\subsection{Framing and more general Riemann surfaces}

The fermionic formulation of the vertex in the standard framing is very easily generalizable to other framings and more general Riemann surfaces.

Consider a Riemann surface with punctures $P_{i}, i=1,2, \ldots$. The punctures are defined by

$$
p_{i}=c_{i} u+d_{i} v+t_{i} \rightarrow 0
$$

and come with coordinates

$$
q_{i}=a_{i} u+b_{i} v \rightarrow \infty
$$

Where $a_{i} d_{i}-b_{i} c_{i}=1$, for integers $a_{i}, b_{i}, c_{i}, d_{i}$. The parameters $t_{i}$ are complex structure moduli of the Riemann surface. According to the discussion above, $\left[q_{i}, p_{i}\right]=-g_{s}$, and

$$
p_{i}=g_{s} \frac{\partial}{\partial q_{i}} .
$$

The coordinates $\left(q_{i}, p_{i}\right)$ at different punctures $P_{i}$ are related to each other by $S L(2, \mathbf{Z})$ transformations, up to constant shifts depending on the $t$ 's. The corresponding Ward identities are given by

$$
\oint_{P_{i}} \psi^{*}\left(q_{i}\right) e^{n q_{i}} \psi\left(q_{i}\right)+\sum_{j \neq i} \oint_{P_{j}} \psi^{*}\left(q_{j}\right) e^{-n\left(A_{i j} q_{j}+B_{i j} p_{j}+T_{i j}\right)} \psi\left(q_{j}\right)=0
$$

where

$$
q_{i}=A_{i j} q_{j}+B_{i j} p_{j}+T_{i j}(t) .
$$

For example, consider the topological vertex in arbitrary framing $(k, l, m)$, i.e. where the coordinates in the $u, v$ and $w$ patches are

$$
q_{u}=u-k v, \quad q_{v}=v-l w, \quad q_{w}=w-m u
$$

respectively. The corresponding momenta are unchanged. We thus have

$$
\oint_{q_{u}} \psi^{*} e^{n q_{u}} \psi+(-1)^{n} \oint_{q_{v}} \psi^{*} e^{n\left(a_{v} q_{v}+b_{v} g_{s} \frac{\partial}{\partial q_{v}}\right)} \psi+(-1)^{n k} \oint_{q_{w}} \psi^{*} e^{n\left(a_{w} q_{w}+b_{w} g_{s} \frac{\partial}{\partial q_{w}}\right)} \psi=0 .
$$


where $a_{v}=-k-1, b_{v}=-1+l-k l, a_{w}=k$, and $b_{w}=1+k+m k$, and similarly for the other charges. The solutions to this correspond to the framed vertex amplitude, as we have verified in examples.

The Ward identity (5.43) is expected to govern the topological string amplitudes on general local toric Calabi-Yau geometries. A toric diagram with $g$ closed meshes is associated to a Riemann surface $H(u, v)=0$ of genus $g$. In these cases the topological string amplitudes are much more non-trivial as one has irreducible curves of various genera at generic degrees. This is reflected in the dependence on the Kähler parameter $t_{i}$, which enter the periods of $\lambda$ on $H(u, v)=0$.

As an example we check (5.43) for the $\mathcal{O}(-3) \rightarrow \mathbf{P}^{2}$ A-model geometry whose B-model curve is genus one with three punctures

$$
H\left(e^{u}, e^{v}\right)=r\left(e^{-t}\right)-e^{-u}-e^{v}+e^{-t+u-v}=0
$$

Here $(t, u, v)$ are the flat A-model moduli. The open/closed mirror map is encoded in $r\left(e^{-t}\right)=\left(\frac{e^{-t}}{z\left(e^{-t}\right)}\right)^{\frac{1}{3}}=1-2 e^{-t}+5 e^{-2 t}+\ldots$ as determined by periods of $\lambda$ on $H=0$ [56]. The choice of the coordinate $q_{i}$ at the punctures is a choice of framing and the one, which corresponds to framing $(0,0,0)$ from gluing three vertices $[9]^{9}$

$$
\begin{aligned}
& Z_{\mathbf{P}^{2}}\left(V_{1}, V_{2}, V_{3}\right)= \\
& \sum_{R_{i}, Q_{i}} C_{Q_{3} R_{2} R_{3}^{t}} C_{Q_{2} R_{1} R_{2}^{t}} C_{Q_{1} R_{3} R_{1}^{t}}(-1)^{\sum_{i} \ell\left(R_{i}\right)} e^{-\sum_{i} \ell\left(R_{i}\right) t} q^{\sum_{i} \kappa_{R_{i}}} \operatorname{Tr}_{Q_{1}} V_{1} \operatorname{Tr}_{Q_{2}} V_{2} \operatorname{Tr}_{Q_{3}} V_{3}
\end{aligned}
$$

is given by

$$
\begin{aligned}
& \left(q_{1}, p_{1}\right)=(-u, u+v+i \pi) \\
& \left(q_{2}, p_{2}\right)=(u-v-t, 2 u-v-t) \\
& \left(q_{3}, p_{3}\right)=(v, 2 v-u+t)
\end{aligned}
$$

The relation between the boundary coordinates is

$$
\left(q_{1}, p_{1}\right)=\left(q_{2}-p_{2},-3 q_{2}+2 p_{2}-t\right)=\left(-2 q_{3}+p_{3}-t, 3 q_{3}-p_{3}+t\right)
$$

yielding the Ward identity

$$
\oint_{P_{1}} \psi^{*}\left(q_{1}\right) e^{n q_{1}} \psi\left(q_{1}\right)+\oint_{P_{2}} \psi^{*}\left(q_{2}\right) e^{n q_{2}-n p_{2}} \psi\left(q_{2}\right)+\oint_{P_{3}} \psi^{*}\left(q_{3}\right) e^{-2 n q_{3}+n p_{3}-n t} \psi\left(q_{3}\right) .
$$

\footnotetext{
9 Note that we exchanged $Q_{1}$ and $Q_{2}$ w.r.t. 9].
} 
The simplest check is performed by choosing $\hat{q}_{2}=q_{2}-p_{2}$, which corresponds to framing $(0,-1,0)$. In particular the action of (5.48) on $Z$ in the $q_{1}, \hat{q}_{2}, q_{3}$ coordinates yields for this choice of framing

$$
\left.\frac{\partial}{\partial t_{n}^{1}} \mathcal{F}\right|_{t_{i}^{3}=0}+\left.\frac{\partial}{\partial t_{n}^{2}} \mathcal{F}\right|_{t_{i}^{3}=0}=0 .
$$

The identity (5.48) does not fix the amplitude as (5.28) does for the vertex, because of the positive power in the second patch. However it can be used to reconstruct the amplitude for three stacks of branes from the one for one stack. This is related to the fact that the identities hold for any fermion number flux, and thus any superposition of fermion number flux in the loop will solve the constraints.

The expression (5.45) yields for framing $(0,0,0)$ up to order $Q=e^{-t}$ and two holes after transforming in the winding basis

$$
\begin{aligned}
F=\sum_{n} & \frac{1}{[n]}\left(t_{n}^{(1)}+t_{n}^{(2)}+t_{n}^{(3)}\right)+Q\left(-\frac{3}{[1]^{2}}-\frac{2}{[1]}\left(t_{1}^{(1)}+t_{1}^{(2)}+t_{1}^{(3)}\right)\right. \\
& \left.-\frac{1}{2}\left(t_{1}^{(1)}\right)^{2}-\frac{[2]}{[1]^{2}}\left(t_{2}^{(1)}+t_{2}^{(2)}+t_{2}^{(3)}\right)-t_{1}^{(1)} t_{1}^{(2)}-t_{1}^{(1)} t_{1}^{(3)}-t_{1}^{(2)} t_{1}^{(3)}\right)+\ldots .
\end{aligned}
$$

Using the normal ordering description (5.28) we can use this to make a first check on (5.48). We have checked (5.48) up to order $Q^{5}$ and 5 holes involving all three stacks of branes. Consistency of (5.28) follows also from the sewing prescription of the amplitudes and the fact that the constraints commute with this operation.

\subsection{Fermion number flux and the topological vertex}

We can illustrate the considerations in section 4 on the fermion number flux also by considering the topological string amplitudes in $\mathcal{O}(-3) \rightarrow \mathbf{P}^{2}$.

We can then introduce a new variable $\theta$ corresponding to turning on flux through the nontrivial cycle, and consider the partition function $Z_{N}$ where $N$ units of flux have been turned on. The computation of these partition functions can be done by using the topological vertex and the gluing constructions, as follows. As explained in [9], the propagator associated to an edge is in general of the form

$$
\delta_{R_{1} R_{2}^{t}}(-1)^{\ell\left(R_{1}\right)(f+1)} q^{f \kappa_{R_{1}} / 2} e^{-\ell(R) t}
$$

where $f$ is a framing which depends on the details of the geometry, and $t$ is the Kähler parameter. This propagator corresponds to setting the momenta in the loops to zero, but 
it is easy to construct a state in $\mathcal{H}^{(1)} \otimes \mathcal{H}^{(2)},\left\langle P^{(f)}\right|$, which includes all possible fermion numbers and is such that $\left\langle P^{(f)} \mid R_{1}\right\rangle \otimes\left|R_{2}\right\rangle$ equals (5.51). This propagator has the form

$$
\left\langle P^{(f)}\right|=\left\langle 0_{12}\right| \exp \left\{\sum_{n=0}^{\infty} e^{-n t-t / 2}\left(e^{i \theta} \epsilon_{n} \psi_{n+1 / 2}^{1 *} \psi_{n+1 / 2}^{2}+e^{-i \theta} \epsilon_{n}^{\prime} \psi_{n+1 / 2}^{1} \psi_{n+1 / 2}^{2 *}\right)\right\}
$$

and it is a bilinear in fermions. The coefficients $\epsilon_{n}, \epsilon_{n}^{\prime}$ are given by:

$$
\begin{aligned}
& \epsilon_{n}=i^{1+f}(-1)^{(1+f) n} q^{\frac{f}{2} n(n+1)}, \\
& \epsilon_{n}^{\prime}=i^{1+f}(-1)^{(1+f) n} q^{-\frac{f}{2} n(n+1)} .
\end{aligned}
$$

Notice as well that the propagator (5.52) is of the form

$$
\left\langle P^{(f)}\right|=\sum_{N \in \mathbf{Z}} e^{i N \theta} \mathcal{O}_{N}\langle N,-N|
$$

where $N$ is the fermion charge, and $\mathcal{O}_{N}$ are operators in the fermion modes, so it includes all possible fermion numbers.

Let us now consider the computation of the partition function with zero fermion number flux associated to local $\mathbf{P}^{2}$. The topological vertex computation is [9]

$$
Z_{0}=\sum_{R_{1}, R_{2}, R_{3}}(-1)^{\sum_{i} \ell\left(R_{i}\right)} e^{-\sum_{i} \ell\left(R_{i}\right) t} q^{\sum_{i} \kappa_{R_{i}}} C_{R_{2} R_{3}^{t}} C_{R_{1} R_{2}^{t}} C_{\cdot R_{3} R_{1}^{t}}
$$

and it is easy to see that the full answer is given by

$$
Z=\sum_{N \in \mathbf{Z}} e^{i N \theta} Z_{N}=\left\langle 0_{159}\right| \otimes\left\langle P_{27}^{(2)}\left|\otimes\left\langle P_{34}^{(-2)}\left|\otimes\left\langle P_{86}^{(2)} \mid V_{123}\right\rangle \otimes\right| V_{546}\right\rangle \otimes\right| V_{987}\right\rangle
$$

where $|V\rangle$ is given by (5.32), with the coefficients of the bilinear in fermions given in (5.40). An explicit computation up to order 2 in $e^{-t}$ shows that indeed, up to an overall constant,

$$
Z_{N= \pm 1}\left(e^{-t}\right)=Z_{0}\left(-q^{ \pm 3} e^{-t}\right)
$$

This is in agreement with (4.17), since the intersection number is precisely 3 for this geometry (the sign in the r.h.s. of (5.54) is due to a quantum shift).

Notice that for example the open string amplitudes for local $\mathbf{P}^{2}$ with outer branes, computed in [9], do not correspond to tau functions of the n-KP hierarchy since they only involve the zero fermion number sector. In order to obtain amplitudes which are Bogoliubov transforms of the vacuum, we have to use in the gluing rules the propagator (5.52), which includes all sectors. 


\section{Connections with Non-critical Strings}

It was already shown in 8$]$ that $c=1$ non-critical bosonic string at self-dual radius is equivalent to topological B-model of the conifold. The rationale for the appearance of the conifold can be viewed as the fact that the holomorphic functions of this CY can be viewed as the ground ring of $c=1$ at self-dual radius [3]. It was further conjectured in [4] that non-critical bosonic string coupled to the $(r, s)$ minimal model is equivalent to the topological string on the $(r, s)$ generalization of the conifold, as reviewed in this paper. The fact that we can directly solve for the amplitudes of these models using the B-model symmetries is in line with the fact that the symmetries of the ground ring of these theories and their deformation encode all the scattering amplitudes. Thus our approach can be viewed as a method to solve non-critical bosonic string by quantizing the deformations of the ground ring. One should identify the B-branes we have considered with the branes of non-critical bosonic strings. This would be interesting to do in detail because of the fact that we have found fermions/branes to play a key role in computing the amplitudes.

In the context of topological B-models, we have seen that there are far more cases that do correspond to a string theory than would be naively expected based on the experience with non-critical bosonic strings. In particular any matrix model in the 't Hooft sense is a string theory equivalent to a topological B-model on some Calabi-Yau [4]. Moreover also these backgrounds do admit 'scattering states' that correspond to deformations at infinity and that are captured by the free energy $\mathcal{F}\left(t_{n}^{i}\right)$. This broadens the class of interesting string theories to study, and frees us of having to take a double scaling limit to discuss a string theory 10 .

More recently there has been some study of non-critical superstring backgrounds (see, for example, [57]). All these models have been related to certain limits of matrix models. Since, as we noted above any matrix model is equivalent to a topological B-models, so does any limit of them. Thus we find, at least at the level of perturbative string theory, a way to go from non-critical bosonic strings to non-critical superstring: One simply considers a given matrix model, which is equivalent to B-model topological string and takes various limits to get one or the other background.

10 It would be interesting to go backwards and see if one can give an interpretation of all these local B-models in terms of non-critical bosonic strings. For example it is natural to speculate that the topological vertex corresponds to non-critical bosonic strings propagating on a geometry of dimension 1 consisting of three half circles connected at two points to one another. 
One main recent motivation to study non-critical superstring has been to find a nonperturbatively complete string theory. In this context it is natural to ask what a nonperturbative definition of topological string may be. This would be interesting to study further.

\section{Open Questions}

We have seen that the Ward identities leading to the solution of the B-model can be formulated in terms of fermions whose wavefunctions transform with suitable Fourier transforms from patch to patch, encoded by the Riemann surface $H(p, x)=0$ where we view $x, p$ as conjugate variables. It would be important to better understand the meaning of this fact: Why are fermions, which correspond to branes, not geometrical objects of the usual kind on the Riemann surface? Moreover why is it that the fermions are free and that the amplitudes can be given by suitable Wick contraction between patches?

Naively one would think that as we move branes from one position to the other the partition function is a smooth function of the position. Let $Z(x)$ denote the partition function of the topological string with a brane at position $x$. Let $x=x_{i}$ denote the asymptotic infinities of the geometry. Let us start with a brane at $x$ near a given $x_{i}$ and move to another one where $x$ approaches $x_{j}$. The statement we have found is that $Z$ transforms as a Fourier transform instead of analytic continuation as we go from $x_{i} \rightarrow x_{j}$. We believe this may be related to an open string holomorphic anomaly. Namely, the actual partition function not only depends on $x$ but also on the complex conjugated variable $\bar{x}$. Thus each brane is associated to a particular asymptotic infinity where the corresponding $\bar{x} \rightarrow \bar{x}_{i}$. Let us define

$$
Z_{i}(x)=Z\left(x, \bar{x}_{i}\right) \quad Z_{j}(x)=Z\left(x, \bar{x}_{j}\right)
$$

There is no reason that these are the same functions, in other words

$$
Z_{i}(x) \neq Z_{j}(x)
$$

thus analytic continuation does not need to work. To find the relation between these two function we need to study

$$
Z_{i}(x)-Z_{j}(x)=\int_{\bar{x}_{i}}^{\bar{x}_{j}} d \bar{x} \bar{\partial} Z(x, \bar{x})
$$


There is an analog of holomorphic anomaly for open string [2] which has not been fully developed. In the closed string case an interpretation of it [11] led to the formulation of the closed string amplitudes as quantum mechanical wavefunctions on the moduli space of Calabi-Yau. Here we want to speculate that there is a similar story in the open string context leading to the amplitudes being wave functions on the Calabi-Yau itself; that there could be roughly a heat-kernel-like equation of the form

$$
\bar{\partial} Z=\left(a g_{s}^{2} \partial^{2}+b g_{s} x \partial+c x^{2}\right) Z
$$

Viewing $p$ as conjugate to $x$ this is the same as the action

$$
\bar{\partial} Z=\left(a p^{2}+b x p+c x^{2}\right) Z
$$

This would imply that an infinitesimal change in the antiholomorphic coordinates generates a linear symplectic diffeomorphism

$$
\begin{aligned}
& x \rightarrow x+(2 a p+b x) \\
& p \rightarrow p-(2 c x+b p) .
\end{aligned}
$$

Exponentiating this action would then generate the metaplectic action on $Z$. It would be important to develop this picture further, if only because it suggests an interesting way to generate the quantum mechanical transformation rules from string theory. It would also

lead to a justification of the procedure followed in this paper, of treating branes/fermions as wavefunctions.

\section{Appendix A. $c=1$ scattering amplitude}

Here we show in detail how the interpretation of the $c=1$ scattering matrix as a Fourier transform corresponds to the familiar scattering amplitudes. Starting from the well-known answer

$$
R_{q}=i\left(\frac{1+i e^{-\pi(\mu+i q)}}{1-i e^{-\pi(\mu+i q)}}\right)^{\frac{1}{2}}\left(\frac{\Gamma\left(\frac{1}{2}-i \mu+q\right)}{\Gamma\left(\frac{1}{2}+i \mu-q\right)}\right)^{\frac{1}{2}}
$$

we can use the identity

$$
\Gamma(x) \Gamma(1-x)=\frac{\pi}{\sin (\pi x)}
$$


to write $R_{q}$ for $q=n+\frac{1}{2}$ as

$$
\begin{aligned}
R_{n+\frac{1}{2}}^{2} & =-\frac{1}{\pi} \frac{1-e^{-i \pi(n-i \mu+1)}}{1+e^{-i \pi(n-i \mu+1)}} \cdot \sin (\pi(n-i \mu+1)) \cdot \Gamma(n-i \mu+1)^{2} \\
& =-\frac{1}{2 \pi i}\left(e^{i \pi(n-i \mu+1) / 2}-e^{-i \pi(n-i \mu+1) / 2}\right)^{2} \Gamma(n+i \mu+1)^{2}
\end{aligned}
$$

So that

$$
R_{n+\frac{1}{2}}=\frac{1}{\sqrt{2 \pi}} e^{i \pi / 4}\left(e^{i \pi(n-i \mu+1) / 2}-e^{-i \pi(n-i \mu+1) / 2}\right) \Gamma(n+i \mu+1)
$$

On the other hand, the expression

$$
\frac{1}{\sqrt{2 \pi}} \int_{-\infty}^{+\infty} d x \cdot x^{n} \cdot x^{-i \mu} e^{i x}
$$

can be evaluated by deforming the contour, by $x \rightarrow i x$ for $x>0$ and to $x \rightarrow-i x$ for $x<0$. (This depends on the sign of $n$, or more generally on where the poles are.) This will turn the Fourier transform into two Laplace transforms, and gives the result

$$
\frac{1}{\sqrt{2 \pi}}\left(e^{i \pi(n-i \mu+1) / 2}-e^{-i \pi(n-i \mu+1) / 2}\right) \Gamma(n-i \mu+1)=e^{-i \pi / 4} R_{n+\frac{1}{2}}
$$

\section{Appendix B. Topological vertex: Solutions to $W_{\infty}$ identities}

Here we give explicit formulas for the free energy of the vertex, obtained by using the $\mathcal{W}_{\infty}$ identities, up to - and including - terms which are cubic in the couplings $t_{n}^{i}$ (i.e. up to three holes). The resulting expressions are exact in $q$ and include the contributions to all genera.

The starting point of the iteration in $s$ is given by (5.29). In order to proceed, we note the following two formulae:

$$
\begin{gathered}
\exp \left\{\sum_{k>0}-\frac{[n k]}{k[k]} z^{k}\right\}=\sum_{k=0}^{n}(-1)^{k}\left[\begin{array}{l}
n \\
k
\end{array}\right] z^{k}, \\
z^{n} \exp \left\{\sum_{k>0} \frac{[n k]}{k[k]} z^{k}\right\}=\sum_{m=n}^{\infty} \frac{[n]}{[m]}\left[\begin{array}{c}
m \\
n
\end{array}\right] z^{m} .
\end{gathered}
$$

These can be easily proved by considering symmetric polynomials in $n$ variables $x_{i}, i=$ $1, \cdots, n$, with the specialization $x_{i}=q^{i-(n+1) / 2}$. The generating function of elementary symmetric functions $E(z)=\prod_{i=1}^{n}\left(1+x_{i} z\right)$ can be computed by induction to be

$$
E(z)=\sum_{k=0}^{n}\left[\begin{array}{l}
n \\
k
\end{array}\right] z^{k},
$$


which is the r.h.s. of the first equation. It is a well-known fact in the theory of symmetric functions that $E(-z)=e^{-P(z)}$, with $P(z)=\sum_{k>0} p_{k} z^{k} / k$, and $p_{k}=\sum_{i=1}^{n} x_{i}^{k}$ (see for example [58]). With the above specialization, we find $p_{k}=[n k] /[k]$, and the first equality (B.1) follows. The second equality follows in a similar way by considering the generating function of complete symmetric functions $H(z)=\prod_{i=1}^{n}\left(1-x_{i} z\right)^{-1}$.

We will now consider amplitudes with $h>1$. The first thing to notice is that the vanishing of $\mathcal{F}_{h>1}$ with a single stack of D-branes allows us to solve for the part of $\mathcal{F}$ of the form $t^{w} \cdots t^{w} t^{u}$ and $t^{v} \cdots t^{v} t^{u}$ to all genera. This involves in principle all terms of the form $\left.\partial_{q_{1}}^{v} \cdots \partial_{q_{\ell}}^{v} \mathcal{F}\right|_{t=0}$ and $\left.\partial_{q_{1}}^{w} \cdots \partial_{q_{\ell}}^{w} \mathcal{F}\right|_{t=0}$, but from (5.29) we know them all and only $\left.\partial_{q}^{w} \mathcal{F}\right|_{t=0}$ is non-vanishing. Using now (B.1) and the value (5.29), we find that the $w$ integral in (5.28) leads to

$$
\begin{aligned}
& -\oint_{w} \frac{d w}{[n]} e^{-\sum_{k>0} \frac{[n k]}{k[k]} e^{-k w}+g_{s}^{-1}[n k] t_{k}^{w} e^{k w}+g_{s} \frac{[n k]}{k} \partial_{k}^{w} \mathcal{F}+\ldots}= \\
& =\sum_{n, m, r} \frac{g_{s}^{-r}}{r !} \sum_{\sum_{i} q_{i}=m} \frac{(-1)^{m+1}}{[n]}\left[\begin{array}{c}
n \\
m
\end{array}\right] \prod_{i=1}^{r}\left[n q_{i}\right] t_{q_{i}}^{w}+\cdots
\end{aligned}
$$

It then follows that the part of $\mathcal{F}$ of the form $t^{w} \cdots t^{w} t^{u}$ is

$$
\sum_{n, m, r} \frac{g_{s}^{-r-1}}{r !} \sum_{\sum_{i} q_{i}=m} \frac{(-1)^{m+1}}{[n]}\left[\begin{array}{c}
n \\
m
\end{array}\right] \prod_{i=1}^{r}\left[n q_{i}\right] t_{q_{i}}^{w} t_{n}^{u}+\text { cyclic }
$$

where "cyclic" denotes the terms obtained by cyclic permutations of the times $t^{u} \rightarrow t^{v} \rightarrow$ $t^{w}$. Similarly one finds in the $v$-patch the part of $\mathcal{F}$ of the form $t^{v} \cdots t^{v} t^{u}$

$$
\sum_{n, m, r} \frac{g_{s}^{-r-1}}{r !} \sum_{\sum_{i} q_{i}=m} \frac{(-1)^{n+r}}{[m]}\left[\begin{array}{c}
m \\
n
\end{array}\right] \prod_{i=1}^{r}\left[n q_{i}\right] t_{q_{i}}^{v} t_{n}^{u}+\text { cyclic. }
$$

Notice that, in particular, the free energy at two holes is given by

$$
\mathcal{F}_{h=2}=g_{s}^{-2} \sum_{n \geq k}(-1)^{k+1} \frac{[n k]}{[n]}\left[\begin{array}{l}
n \\
k
\end{array}\right]\left(t_{n}^{u} t_{k}^{w}+\text { cyclic }\right) .
$$

We can now compute cubic terms of the form $t_{p}^{w} t_{m}^{u} t_{n}^{u}$. In that case, the only contribution to $\partial_{n}^{u} \mathbf{C} F$ that we need to consider comes from products of $t_{k}^{w}$ with arbitrary derivatives of $\mathcal{F}$,

$$
-\sum_{p, k}[n p](-1)^{k}\left[\begin{array}{l}
n \\
k
\end{array}\right] t_{p}^{w} \sum_{r} \frac{g_{s}^{r-1}}{r !} \sum_{\sum_{i} q_{i}=p-k} \prod_{i=1}^{r} \frac{\left[n q_{i}\right]}{q_{i}} \partial_{q_{1}}^{w} \cdots \partial_{q_{r}}^{w} \mathcal{F}
$$


where we consider the contribution to the multiple derivative of (B.4). The final expression for the coefficient of $t_{p}^{w} t_{m}^{u} t_{n}^{u}$ is:

$$
g_{s}^{-3}(-1)^{p} \frac{[n p]}{[n][m]} \sum_{r=1}^{p} \sum_{k=0}^{p-r} \frac{1}{r !}\left[\begin{array}{l}
n \\
k
\end{array}\right]\left[\begin{array}{c}
m \\
p-k
\end{array}\right] \sum_{\sum_{i=1}^{r} q_{i}=p-k} \prod_{i=1}^{r} \frac{\left[n q_{i}\right]\left[m q_{i}\right]}{q_{i}} t_{n}^{u} t_{m}^{u} t_{p}^{w}
$$

Of course, if $n=m$ there is an extra $1 / 2$. We have checked in many cases that this complicated albeit explicit expression adds up to a simple result which is manifestly symmetric in $n, m$ :

$$
(-1)^{p} g_{s}^{-3} \frac{[n p][m p]}{[n+m]}\left[\begin{array}{c}
n+m \\
p
\end{array}\right] t_{n}^{u} t_{m}^{u} t_{p}^{w}
$$

whose genus zero limit is

$$
(-1)^{p} g_{s}^{-2} \frac{n m p^{2}}{n+m}\left(\begin{array}{c}
n+m \\
p
\end{array}\right)
$$

Let us now consider the coupling $t_{p}^{w} t_{m}^{v} t_{n}^{u}$. This has two contributions, one coming from the $w$ patch, and the other from the $v$ patch. For the $w$ patch, we start again from (B.3), but now have to consider the piece of the multiple derivative that is proportional to $t^{v}$. This is obtained from (B.5), and we find for the coefficient of $t_{n}^{u} t_{m}^{v} t_{p}^{w}$

$$
a(n, m, p)=-g_{s}^{-3} \frac{[n p]}{[n]} \sum_{r=1}^{p} \sum_{k=0}^{p-r} \frac{1}{r !} \frac{(-1)^{k+r+m}}{[p-k]}\left[\begin{array}{c}
n \\
k
\end{array}\right]\left[\begin{array}{c}
p-k \\
m
\end{array}\right] \sum_{\sum_{i=1}^{r} q_{i}=p-k} \prod_{i=1}^{r} \frac{\left[n q_{i}\right]\left[m q_{i}\right]}{q_{i}}
$$

The contribution from the $v$ patch is obtained in a similar way, and one finds

$$
b(n, m, p)=g_{s}^{-3} \frac{[n m]}{[p]} \sum_{r=1}^{m-1} \sum_{k=n}^{m-r} \frac{1}{r !} \frac{(-1)^{k+n+m+r}}{[k]}\left[\begin{array}{l}
k \\
n
\end{array}\right]\left[\begin{array}{c}
p \\
m-k
\end{array}\right] \sum_{\sum_{i=1}^{r} q_{i}=m-k} \prod_{i=1}^{r} \frac{\left[p q_{i}\right]\left[n q_{i}\right]}{q_{i}}
$$

The coefficient of $t_{n}^{u} t_{m}^{v} t_{p}^{w}$ in $\mathcal{F}\left(g_{s}, t_{n}^{i}\right)$ is then given by

$$
F_{n m p}^{u v w}=a(n, m, p)+b(n, m, p) .
$$

Explicit evaluations show that the above coefficient is cyclically symmetric in $n, m, p$, as it should be. All the results obtained here for $h=2,3$ holes and arbitrary winding numbers $n, m, p$ are in perfect agreement with the free energy as computed from (5.17). 


\section{Acknowledgments}

We would like to thank N. Arkani-Hamed, J. de Boer, S. Cherkis, S. Gukov, C. Lazaroiu, W. Lerche, I. Low, J. McGreevy, G. Moore, A. Neitzke, L. Randall, T. Takayanagi and E. Verlinde for useful conversations.

The research of M.A. was supported in part by DOE grant DE-FG02-96ER40956 and by a DOE OJI Award. The research of C.V. was supported in part by NSF grants PHY0244821 and DMS-0244464. The research of R.D. was partly supported by FOM and the CMPA grant of the University of Amsterdam. In addition, CV thanks the hospitality of the theory group at Caltech, where he was a Gordon Moore Distinguished Scholar. A.K. is supported in part by the DFG grant KL-1070/2-1. M.M. would like to thank the High Energy Theory group of the University of Washington at Seattle for hospitality during part of this work. 


\section{References}

[1] R. Dijkgraaf, "Intersection theory, integrable hierarchies and topological field theory," in J. Frohlich et al. (eds), New symmetry principles in quantum field theory, Plenum Press, p. 95, arXiv:hep-th/9201003.

[2] M. Bershadsky, S. Cecotti, H. Ooguri and C. Vafa, "Kodaira-Spencer theory of gravity and exact results for quantum string amplitudes," hep-th/9309140, Commun. Math. Phys. 165 (1994) 311.

[3] E. Witten, "Ground ring of two-dimensional string theory," Nucl. Phys. B 373, 187 (1992) arXiv:hep-th/9108004.

[4] R. Dijkgraaf and C. Vafa, "Matrix models, topological strings, and supersymmetric gauge theories," Nucl. Phys. B 644, 3 (2002) arXiv:hep-th/0206255.

[5] R. Dijkgraaf and C. Vafa, "On geometry and matrix models," Nucl. Phys. B 644, 21 (2002) arXiv:hep-th/0207106.

[6] I. K. Kostov, "Conformal field theory techniques in random matrix models," arXiv:hep-th/9907060.

[7] S. Chiantese, A. Klemm, I. Runkel, "Higher order loop equations for A(r) and D(r) quiver matrix models," arXiv:hep-th/0311258.

[8] D. Ghoshal and C. Vafa, " $c=1$ string as the topological theory of the conifold," Nucl. Phys. B 453, 121 (1995) arXiv:hep-th/9506122.

[9] M. Aganagic, A. Klemm, M. Mariño and C. Vafa, "The topological vertex," arXiv:hepth/0305132.

[10] K. Hori and C. Vafa, "Mirror symmetry," hep-th/0002222.

[11] E. Witten, "Quantum background independence in string theory," arXiv:hepth/9306122].

[12] R. Dijkgraaf, G. W. Moore and R. Plesser, "The Partition function of 2-D string theory," Nucl. Phys. B 394, 356 (1993) arXiv:hep-th/9208031].

[13] E. Calabi, "Métriques Kählériennes et Fibrés Holomorphes," Ann. scient. Éc. Norm. Sup. (1979) 294, G. Tian and S.-T. Yau, "Existence of Kähler-Einstein Metrics on complete Kähler manifolds and their applications to algebraic geometry," Mathematical Aspect of String Theory, 1986 at UCSD, Ed. S.T. Yau, World Scientific (1987), "Complete Kähler manifolds with zero Ricci curvature, I," Journal of AMS 3 (1990), "Complete Kähler manifolds with zero Ricci curvature, II," Inv. Math. 106 (1991).

[14] M. Aganagic and C. Vafa, "Mirror symmetry, D-branes and counting holomorphic discs," arXiv:hep-th/0012041.

[15] C. Vafa, "Brane/anti-brane systems and U(N|M) supergroup," arXiv:hep-th/0101218.

[16] E. Witten, "Five-brane effective action in M-theory," J. Geom. Phys. 22, 103 (1997) arXiv:hep-th/9610234. 
[17] N. Nekrasov and A. Okounkov, "Seiberg-Witten theory and random partitions," arXiv:hep-th/0306238.

[18] A. Iqbal and A. Okounkov, private communication.

[19] C.-C. M. Liu, K. Liu and J. Zhou, "A proof of a conjecture of Mariño-Vafa on Hodge integrals," math.AG/0306434; "A formula of two-partition Hodge integrals," math.AG/0310272.

[20] I. Low and A. V. Manohar, "Spontaneously broken spacetime symmetries and Goldstone's theorem," Phys. Rev. Lett. 88, 101602 (2002) arXiv:hep-th/0110285.

[21] R. Gopakumar and C. Vafa, "On the gauge theory/geometry correspondence," Adv. Theor. Math. Phys. 3, 1415 (1999) arXiv:hep-th/9811131.

[22] F. Cachazo, K. A. Intriligator and C. Vafa, "A large N duality via a geometric transition," Nucl. Phys. B 603, 3 (2001) arXiv:hep-th/0103067.

[23] M. Fukuma, H. Kawai and R. Nakayama, "Continuum Schwinger-Dyson Equations And Universal Structures In Two-Dimensional Quantum Gravity," Int. J. Mod. Phys. A 6, 1385 (1991), "Infinite Dimensional Grassmannian Structure Of Two-Dimensional Quantum Gravity," Commun. Math. Phys. 143, 371 (1992).

[24] C. Itzykson and J. B. Zuber, "The Planar Approximation. 2," J. Math. Phys. 21, 411 (1980).

[25] H. Ooguri and C. Vafa, "Knot invariants and topological strings," Nucl. Phys. B 577, 419 (2000) arXiv:hep-th/9912123.

[26] A. Neitzke and C. Vafa, work in progress.

[27] R. Dijkgraaf, H. Verlinde and E. Verlinde, "Loop Equations And Virasoro Constraints In Nonperturbative 2-D Quantum Gravity," Nucl. Phys. B 348, 435 (1991).

[28] F. Cachazo, S. Katz and C. Vafa, "Geometric transitions and N = 1 quiver theories," arXiv:hep-th/0108120.

[29] F. Cachazo, B. Fiol, K. A. Intriligator, S. Katz and C. Vafa, "A geometric unification of dualities," Nucl. Phys. B 628, 3 (2002) arXiv:hep-th/0110028.

[30] S. Kharchev, A. Marshakov, A. Mironov, A. Morozov and S. Pakuliak, "Conformal matrix models as an alternative to conventional multimatrix models," Nucl. Phys. B 404, 717 (1993) arXiv:hep-th/9208044. A. Morozov, "Integrability And Matrix Models," Phys. Usp. 37, 1 (1994) arXiv:hep-th/9303139.

[31] E. Brézin and V. A. Kazakov, "Exactly Solvable Field Theories Of Closed Strings," Phys. Lett. B 236, 144 (1990).

[32] M. R. Douglas and S. H. Shenker, "Strings In Less Than One-Dimension," Nucl. Phys. B 335, 635 (1990).

[33] D. J. Gross and A. A. Migdal, "A Nonperturbative Treatment Of Two-Dimensional Quantum Gravity," Nucl. Phys. B 340, 333 (1990).

[34] M. R. Douglas, "Strings In Less Than One-Dimension And The Generalized KdV Hierarchies," Phys. Lett. B 238, 176 (1990). 
[35] E. Witten, "Two-Dimensional Gravity And Intersection Theory On Moduli Space," Surveys Diff. Geom. 1, 243 (1991).

[36] K. Li, "Topological Gravity With Minimal Matter," Nucl. Phys. B 354, 711 (1991). "Recursion Relations In Topological Gravity With Minimal Matter," Nucl. Phys. B 354, 725 (1991).

[37] R. Dijkgraaf, H. Verlinde and E. Verlinde, "Topological Strings In $d<1$," Nucl. Phys. B 352, 59 (1991).

[38] E. J. Martinec, "Criticality, Catastrophes And Compactifications," in Brink, L. (ed.) et al., Physics and mathematics of strings, World Scientific, p. 389. C. Vafa and N. P. Warner, "Catastrophes And The Classification Of Conformal Theories," Phys. Lett. B 218, 51 (1989).

[39] H. Ooguri and C. Vafa, "Two-Dimensional Black Hole and Singularities of CY Manifolds," Nucl. Phys. B 463, 55 (1996) arXiv:hep-th/9511164].

[40] I. K. Kostov, "String equation for string theory on a circle," Nucl. Phys. B 624, 146 (2002) arXiv:hep-th/0107247. S. Y. Alexandrov, V. A. Kazakov and I. K. Kostov, "Time-dependent backgrounds of 2D string theory," Nucl. Phys. B 640, 119 (2002) arXiv:hep-th/0205079. I. K. Kostov, "Integrable flows in $c=1$ string theory," J. Phys. A 36, 3153 (2003) arXiv:hep-th/0208034.

[41] C. Imbimbo and S. Mukhi, "The Topological matrix model of $c=1$ string," Nucl. Phys. B 449, 553 (1995) arXiv:hep-th/9505127.

[42] T. Eguchi and S. K. Yang, "The Topological $\mathbf{C P}^{1}$ model and the large $N$ matrix integral," Mod. Phys. Lett. A 9, 2893 (1994) arXiv:hep-th/9407134]. T. Eguchi, K. Hori and S. K. Yang, "Topological sigma models and large $N$ matrix integral," Int. J. Mod. Phys. A 10, 4203 (1995) arXiv:hep-th/9503017.

[43] A. Okounkov and R. Pandharipande, "The equivariant Gromov-Witten theory of $\mathbf{P}^{1}$," math.AG/0207233.

[44] A. Iqbal and A. K. Kashani-Poor, " $S U(N)$ geometries and topological string amplitudes," arXiv:hep-th/0306032.

[45] D. E. Diaconescu and B. Florea, "Localization and gluing of topological amplitudes," arXiv:hep-th/0309143.

[46] A. Okounkov, N. Reshetikhin and C. Vafa, "Quantum Calabi-Yau and classical crystals," arXiv:hep-th/0309208.

[47] T. Eguchi and H. Kanno, "Topological strings and Nekrasov's formulas," arXiv:hepth/0310235.

[48] T. J. Hollowood, A. Iqbal and C. Vafa, "Matrix models, geometric engineering and elliptic genera," arXiv:hep-th/0310272.

[49] J. Zhou, "Curve counting and instanton counting," math.AG/0311237.

[50] M. Aganagic, M. Mariño and C. Vafa, "All loop topological string amplitudes from Chern-Simons theory," hep-th/0206164. 
[51] L. D. Faddeev and R. M. Kashaev, "Quantum Dilogarithm," Mod. Phys. Lett. A 9, 427 (1994) arXiv:hep-th/9310070.

[52] T. Miwa, M. Jimbo and E. Date, Solitons, Cambridge University Press, 2000.

[53] J. Zhou, "A conjecture on Hodge integrals," math.AG/0310282.

[54] V. G. Kac and J. W. van de Leur, "The n-Component KP Hierarchy And Representation Theory," J. Math. Phys. 44, 3245 (2003) arXiv:hep-th/9308137.

[55] J. Zhou, "Hodge integrals and integrable hierarchies," math.AG/0310408.

[56] M. Aganagic, A. Klemm and C. Vafa, "Disk instantons, mirror symmetry and the duality web," Z. Naturforsch. A 57, 1 (2002) arXiv:hep-th/0105045.

[57] J. McGreevy and H. Verlinde, "Strings from tachyons: The $c=1$ matrix reloaded," arXiv:hep-th/0304224. T. Takayanagi and N. Toumbas, "A matrix model dual of type 0B string theory in two dimensions," JHEP 0307, 064 (2003) arXiv:hep-th/0307083. M. R. Douglas, I. R. Klebanov, D. Kutasov, J. Maldacena, E. Martinec and N. Seiberg, "A new hat for the $c=1$ matrix model," arXiv:hep-th/0307195.

[58] I.G. Macdonald, Symmetric functions and Hall polynomials, Oxford University Press, 1995. 